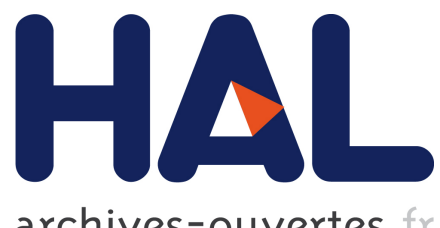

archives-ouvertes

\title{
A variational approach to single crystals with dislocations
}

Riccardo Scala, Nicolas Goethem

\section{To cite this version:}

Riccardo Scala, Nicolas Goethem. A variational approach to single crystals with dislocations. 2018. hal-01671033v3

\section{HAL Id: hal-01671033 https://hal.archives-ouvertes.fr/hal-01671033v3}

Submitted on 21 Dec 2018

HAL is a multi-disciplinary open access archive for the deposit and dissemination of scientific research documents, whether they are published or not. The documents may come from teaching and research institutions in France or abroad, or from public or private research centers.
L'archive ouverte pluridisciplinaire HAL, est destinée au dépôt et à la diffusion de documents scientifiques de niveau recherche, publiés ou non, émanant des établissements d'enseignement et de recherche français ou étrangers, des laboratoires publics ou privés. 


\title{
A variational approach to single crystals with dislocations
}

\author{
Riccardo Scala \\ University of Rome 1, "La Sapienza", \\ Dipartimento di matematica "G. Castelnuovo", \\ Piazzale Aldo Moro 5, 00185 Roma, Italy. \\ scala@mat.uniroma1.it \\ Nicolas Van Goethem \\ Universidade de Lisboa, Faculdade de Ciências, \\ Departamento de Matemática, CMAFcIO, \\ Alameda da Universidade, C6, 1749-016 Lisboa, Portugal \\ vangoeth@fc.ul.pt (TEL:+351-217500486) \\ ORCID: 0000-0002-5356-8383
}

December 21, 2018

\begin{abstract}
We study the graphs of maps $u: \Omega \rightarrow \mathbb{R}^{3}$ whose curl is an integral 1-current with coefficients in $\mathbb{Z}^{3}$. We characterize the graph boundary of such maps under suitable summability property. We apply these results to study a three-dimensional single crystal with dislocations forming general one-dimensional clusters in the framework of finite elasticity. By virtue of a variational approach, a free energy depending on the deformation field and its gradient is considered.

The problem we address is the joint minimization of the free energy with respect to the deformation field and the dislocation lines. We apply closedness results for graphs of torus-valued maps, seen as integral currents and, from the characterization of their graph boundaries we are able to prove existence of minimizers.
\end{abstract}

\section{Contents}

1 Introduction $\quad 2$

$\begin{array}{llr}2 & \text { Model variables } & 7\end{array}$

3 Mathematical formalism $\quad 9$

3.1 Generalities about currents and graphs . . . . . . . . . . . . . . . . 10

3.2 Graphs of maps with values in $\mathbb{T}^{3} \ldots \ldots \ldots \ldots \ldots . \ldots \ldots$

3.3 The displacement and the dislocation-induced deformation: basic results . . 14

3.4 Preliminaries on dislocations at the continuum scale . . . . . . . . . . 16

3.5 Properties of the dislocation-induced deformation . . . . . . . . . . . 17

4 Boundary of graphs $\quad 19$

4.1 Preliminary results . . . . . . . . . . . . . . . . . . . . . . . 19

4.2 Weak form of the graph boundaries . . . . . . . . . . . . . . . 19 
4.3 Case of a single dislocation . . . . . . . . . . . . . . . . 21

4.4 Main result 1: characterization of the graph boundary for clusters with a single Burgers vector . . . . . . . . . . . . . . . . 28

4.5 Main result 2: characterization of the graph boundary for clusters with general Burgers vectors . . . . . . . . . . . . . . 33

5 Minimization problems $\quad \mathbf{3 5}$

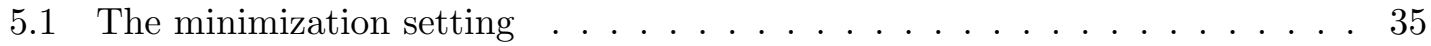

5.2 Problem 1: dislocation clusters with linearly dependent Burgers vectors . . 37

5.3 Problem 2: dislocation clusters with general Burgers vectors . . . . . . . . . 39

5.4 A problem involving $F$ and $F^{-1}$ and modeling discussion . . . . . . . . 40

\section{Introduction}

One of the longstanding problems in the theory of dislocations is to understand the threedimensional configuration of the clusters associated to minimizers of the elastic energy. One of the main difficulty to deal with is the singularity of the stress and strain in the vicinity of dislocation lines, that implies their non-square integrability, as opposed to the traditional models of elasticity. Indeed, the standard approach of regularizing the fields by introducing a small tubular neighborhood of the dislocation core is often adopted, in the framework of linear elasticity. The different approach consisting in proposing an energy functional with less-than quadratic growth in the framework of nonlinear elasticity is here adopted, as suggested in the pioneering paper by Müller and Palombaro [26], itself based on the framework proposed by Ortiz in [28]. Even the mere existence of minimizers for such an energy is a very challenging problem, whose preliminary solutions have been proposed by the authors in a series of papers [31-33]. In these contributions the problem has been generalized as to consider as variables a deformation tensor field and the dislocation density, in contrast with [26] where the dislocation is a fixed circular loop (and hence not subjected to joint optimization together with $F$ ). In the present paper we propose a complete solution of the minimization problem in the case where the dislocation cluster is generated by one Burgers vector. As a refinement of the results of [33], where a strong hypothesis related to a regularizing term in the energy was made, here we relax such hypothesis into a weaker one, that we call "physical" (see the subsequent discussion for details and the content of the main results in Section 5). To prove existence of minimizers, the main mathematical tool used is Federer's theory of currents [17] (with the aid of Cartesian currents theory as well, see, e.g., [21]) to describe both the deformation and the dislocation lines, together with Ball's variational approach to finite elasticity [7]. The use of currents has shown to be very useful recently to model dislocations. Treating these objects as integral currents provides strong closedness principles which are not available for measures, and the notion of convergence for vector-valued measures is too weak to fit. Furthermore, the theory of currents with coefficients in a group $[18,40]$ is used to describe dislocations with Burgers vectors constrained to stay in a prescribed lattice. A consequence of this constraint, as we will see, is that the displacement can equivalently be seen as a torus-valued map, and hence the theory of currents on non-Euclidean spaces [14] turns out to be crucially helpful.

This analysis is a necessary prerequisite to study the evolution of dislocation clusters, in particular at the quasistatic regime. A first contribution as a sequel of this work has been given in [34]. 


\section{The crystal with dislocations in finite elasticity}

A single crystal is represented by a three-dimensional body $\Omega \subset \mathbb{R}^{3}$ with a smooth boundary. We denote by $\mathcal{L} \subset \bar{\Omega}$ the set of dislocation lines. The basic kinematic variable is the tensor $F \in \mathbb{R}^{3 \times 3}$ which represents the state of deformation of the body. The physical interpretation of the chosen kinematical variable $F$ in terms of deformation (to or from the current configuration) is discussed in Section 5.4 as based on the physical insight of Acharya contributions $[1,2,4]$ and sometimes in contrast with [28]. In our approach we prefer not to mention any reference configuration, since this notion is ambiguous in the presence of dislocation singularities. Following Ph. Ciarlet's and coauthors [11] intrinsic approach, we rather consider $F$ as an intrinsic quantity, namely through the differential-geometric metric tensor $C=F^{T} F,\left(C_{i j}=F_{i}^{l} \delta_{l k} F_{j}^{k}\right)$. Further, the basic dislocation variable is its density tensor, i.e., the finite tensor-valued Radon measure

$$
\Lambda_{\mathcal{L}}:=\tau \otimes b \mathcal{H}_{\lfloor\mathcal{L}}^{1}
$$

In this formula $b$ is the Burgers vector, a vector defined on $\mathcal{L}$ and constant on each isolated loop of $\mathcal{L}, \tau$ is the unit tangent vector of the Lipschitz curve $\mathcal{L}$ defined $\mathcal{H}_{\left[\mathcal{L}^{-}\right.}^{1}$ almost everywhere, where $\mathcal{H}_{\mid \mathcal{L}}^{1}$ denotes the one-dimensional Hausdorff measure restricted to $\mathcal{L}$. In our approach the deformation field $F$ and the dislocation density are linked by the constraint

$$
-\operatorname{Curl} F=\Lambda_{\alpha}^{T}
$$

The main problem we study in this work is the minimization of an energy functional $\mathcal{W}$ representing the internal energy of a deformed single crystal in the presence of dislocations. The available free energy $\mathcal{W}$ is assumed to be a function of some invariant (under Euclidean transformations) tensors describing the mechanical as well as the dislocation-induced deformations, the tensor $C$, and the dislocation density $\Lambda_{\mathcal{L}}$. Since the dislocation cluster is a micro-structure, we will also assume that $\mathcal{W}$ depends on an appropriate second-order deformation related to $C$ and the gradient of $C$, called the material connexion $\Gamma{ }^{1}$

\section{The variational problem}

The energy $\mathcal{W}$ depends on $C, \Gamma$, and $\Lambda_{\mathcal{L}}$. In order to address the minimization problem while complying to classical principles of rational mechanics, we assume that the energy is a function of the first- and second-order invariant tensors related to $F$ and $D F$. It is shown in Section 2 how this dependence can be written in terms of $M(F):=(F$, cof $F$, det $F)$ (the vector of all subdeterminants of the matrix $F$ ), $\operatorname{Curl} F$, and $\operatorname{Div} F$. Therefore the energy reads

$$
\mathcal{W}(F, D F)=\mathcal{W}_{\mathrm{e}}(M(F))+\mathcal{W}_{\mathrm{d}}(\operatorname{Div} F)+\mathcal{W}_{\text {dislo }}\left(\Lambda_{\mathcal{L}}\right)
$$

where

$$
\mathcal{W}_{\mathrm{e}}(M(F))=\int_{\Omega} W_{\mathrm{e}}(F(x), \operatorname{cof} F(x), \operatorname{det} F(x)) d x,
$$

\footnotetext{
${ }^{1}$ This object, following [8] (under the physical interpretation that $F$ is the classical deformation tensor from a reference to the current configuration), is the correct thermodynamical quantity to be considered in finite elasticity. The skew-symmetric part of $\Gamma$ is related to the dislocation density tensor introduced by Noll in the context of continuum bodies with dislocations [27]. We emphasize that this geometric object has been used for a long time in the literature, since the works of E. Kröner in the fifties until recently (see for instance $[24,30,36,41])$.
} 
with $\mathcal{W}_{\mathrm{e}}$ lower semicontinuous with respect to the weak convergence of $M(F) ; \mathcal{W}_{\text {dislo }}$ is a functional on the space of Radon measures $\mathcal{M}_{b}\left(\Omega, \mathbb{R}^{3 \times 3}\right)$ which is lower semicontinuous with respect to the weak- $\star$ convergence, and

$$
\mathcal{W}_{\mathrm{d}}(\operatorname{Div} F)=\int_{\Omega} W_{\mathrm{d}}(\operatorname{Div} F(x)) d x,
$$

is a weakly lower semi-continuous functional on $L^{r}\left(\Omega, \mathbb{R}^{3}\right), r \geq 1$. Note that $W_{\mathrm{d}}($ Div $F)$ can be also seen as a perturbation of the energy $\mathcal{W}_{\mathrm{e}}+\epsilon \mathcal{W}_{\text {dislo }}$ for some small parameter $\epsilon$ related to the micro-structure (see [19]). Even if often $W_{\mathrm{e}}$ is taken polyconvex in order to supply for weakly lower semicontinuity, we emphasize that in our minimization problem it can be general, for it suffices to be $L^{1}$-lower semicontinuous in $M(F)$. Let us also remark that this approach allows us to consider an elastic energy that depends on $F$ and $F^{-1}$, as discussed in Section 5.4; the quantity $F^{-1}$ is indeed of crucial meaning in many dislocation classical models (see for instance [1-4]) in contrast with the approach of $[26,28]$ that we follow. Even though the variable $F^{-1}$ can be expressed as combination of the entries of $M(F)$ (namely, $\left.F^{-1}=(\operatorname{cof} F)^{T} / \operatorname{det} F\right)$, at the present stage we cannot address the existence of a minimization problem involving only the variable $F^{-1}$ in the stored-elastic part, since we still need some control on $\operatorname{cof} F$ and $\operatorname{det} F$, and hence energy dependence on these variables (see Section 5.4).

We will make the following crucial coercivity assumption,

$$
\mathcal{W}(F, D F) \geq C\left(\|F\|_{L^{p}}^{p}+\|\operatorname{cof} F\|_{L^{q}}^{q}+\delta\|\operatorname{det} F\|_{L^{s}}^{s}+\|\operatorname{Div} F\|_{L^{r}}^{r}\right)+c\left|\Lambda_{\mathcal{L}}\right|(\Omega)-\gamma
$$

for some constitutive positive constants $\gamma, C$, and $c$, and $\delta \geq 0$. We assume $1<p<2$, $q, r, s>1$ and $-\operatorname{Curl} F=\Lambda_{\mathcal{L}} \in \mathcal{M}_{b}(\Omega)$, a bounded Radon measure. Note that if $\mathcal{W}$ is independent of $\operatorname{det} F$ (and hence $\delta=0$ ), we can infer boundedness of $\operatorname{det} F$ by control of $F$ and $\operatorname{cof} F$.

Let $\Omega \subset \hat{\Omega}$, with $\Omega$ and $\hat{\Omega}$ simply connected and smooth. Let $b \in 2 \pi \mathbb{Z}^{3}$ a fixed Burgers vector. We fix a boundary condition $\hat{\alpha}$ for the dislocation (see Section 5 for the specific notation) and a map $\hat{F} \in L^{p}\left(\hat{\Omega} ; \mathbb{R}^{3 \times 3}\right)$ with $-\operatorname{Curl} \hat{F}=\Lambda_{\alpha}^{T}=b \otimes \hat{\alpha}$ on $\hat{\Omega}$, and Div $\hat{F} \in L^{r}\left(\hat{\Omega} ; \mathbb{R}^{3}\right)$ with $r>1$. Then we define the class of admissible functions as

$$
\begin{aligned}
\mathcal{F}_{b}:=\left\{F \in L^{p}\left(\hat{\Omega} ; \mathbb{R}^{3 \times 3}\right): \operatorname{cof} F \in L^{q}\left(\hat{\Omega} ; \mathbb{R}^{3 \times 3}\right), \operatorname{det} F \in L^{s}(\hat{\Omega}), \operatorname{Div} F \in L^{r}\left(\hat{\Omega} ; \mathbb{R}^{3}\right),\right. \\
\\
\quad-\operatorname{Curl} F=\Lambda_{\mathcal{L}}^{T}=b \otimes \mathcal{L} \text { for some closed integral 1-current } \mathcal{L}, \\
\quad \text { and } F=\hat{F} \text { on } \hat{\Omega} \backslash \Omega\} .
\end{aligned}
$$

We are interested in the variational problem

$$
\min _{F \in \mathcal{F}_{b}} \mathcal{W}(F, D F)
$$

Note that both $F$ and $\mathcal{L}$ are unknown. The following theorem was proved in [33].

Theorem 1.1 (Existence result with regularization term [33]). Let $1<p=q=s<$ $2, r>3$. There exists a solution $F \in \mathcal{F}_{b}$ of the minimum problem (1.3).

We remark that the original problem was formulated in [26] with $\mathcal{L}$ fixed, $F$ assumed to be locally the gradient of a Cartesian map away from $\mathcal{L}$, and with $\mathcal{W}_{\mathrm{d}}=0$ (and $\mathcal{W}_{\text {dislo }}$ constant, the dislocation being fixed). Then, with $F$ and $\mathcal{L}$ unknown, the variational problem was first solved in [32] with $\mathcal{W}_{\mathrm{d}}=0$ but, in place, with an energy term accounting 
either for the number and length of generating dislocation loops, or equivalently, the number of connected components of a 1-set containing $\mathcal{L}$. This kind of assumptions are in the spirit of similar done for variational problems in fracture, see, e.g., [13]. The main novelty in [33] is the replacement of these energetic terms by $\mathcal{W}_{\mathrm{d}}$, the latter having the physical interpretation of a higher-order term related to the invariants of the gradient of the tensor $C$. However it is remarkable the necessity of the condition $r>3$, which is physically unjustified since the natural ambient space for $F$ is $L^{p}$ with $p<2$. In this work we relax this assumption in order to have $r<2$ as well. We call this assumption "physical".

\section{The existence results}

As anticipated, we would like to avoid the rather strong condition $r>3$ in Theorem 1.1 which is presumably too strong with respect to the singular forces exerted by the dislocations. It turns out that if we consider the hypothesis cof $F \in L^{2}$, we are able to weaken considerably the assumption $r>3$ and in particular find a range for the exponent $r$ which accounts, as for $p$, for the case $r<2$. The first existence result of the present paper (see Theorem 5.2 in Section 5) is the following.

Theorem 1.2 (For general dislocation clusters with one Burgers vector). Let $1<p<$ $2, q \geq 2, s>1$, and $r>\frac{12}{7}$. Under suitable hypotheses on the coefficients (see Theorem 5.2) there exists a minimizer $F \in \mathcal{F}_{b}$ of $\mathcal{W}$.

In Section 2 we will see that $F$ can be written as the sum of the gradient of two maps, $u$ and $v$, the first one with values in the 3 -dimensional flat torus $\mathbb{T}^{3}$. The proof of Theorem 1.2 relies on analyzing the graph $\mathcal{G}_{u+v}$ of the deformation maps $u$ and $v$, seen as an integer-multiplicity current. Then, a characterization of the boundary of the graph of $u+v$ is required. Here the theory of currents in metric spaces (the flat torus in our case) and its link with the theory of integral currents with coefficients in $\mathbb{Z}^{3}$ becomes crucial. Specifically, suitable closure and compactness theorems are required; these can be obtained by application of the theory developed by Fleming [18] and the rectifiability property of flat chains [40], together with the adaptation of classical compactness theorems by Federer to the case of metric spaces, whose solid basis has been recently developed by De Pauw and Hardt $[14,15]$. Let us here stress that the use of currents in the space $\Omega \times \mathbb{T}^{3}$ is a direct consequence of the choice $\mathbb{Z}^{3}$ for the lattice where the Burgers vectors are constrained. The dislocation currents are treated as classical flat chains, namely 1-integral currents with coefficients in the group $\mathbb{Z}^{3}$.

A general expression for the boundary of $\mathcal{G}_{u+v}$ is given in Theorem 4.2 of Section 4 , and this expression reduces to a current representing the dislocation density, i.e.,

$$
\partial \mathcal{G}_{u+v} \equiv \mathcal{L} \otimes b,
$$

in the case $q \geq 2$. In the latter case, we will show that, due to the high integrability of cof $F$, in the region close to the dislocation cluster it must hold

$$
\nabla u \times \nabla v=0 .
$$

In the more general case of linearly independent Burgers vectors, a characterization of the graphs $\mathcal{G}_{u+v}$ is currently far from reach, but can be addressed in some specific geometric setting. For instance, it is possible to show that if the three dislocation clusters associated to the Burgers vectors $e_{1}, e_{2}, e_{3}$ are disjoint (which takes place in many observed 
situations, as in the case of parallel dislocation lines, or when the clusters are constrained to lie on parallel slip planes) then the graph $\mathcal{G}_{u+v}$ associated to the deformation $F$ is an integer-multiplicity current (see Theorem 4.13). Moreover, also in this setting, if the cofactor is square integrable, then the boundary $\partial \mathcal{G}_{u+v}$ still coincides with the dislocation density (see Theorem 4.14). This allows us to solve a more general minimization problem. However, since we are yet not able to characterize $\partial \mathcal{G}_{u+v}$ in every geometric setting, we must appeal to an extra hypothesis on the admissible class of deformations. Specifically, we denote by $\mathcal{L}_{i}$ the dislocation current associated to the Burgers vector $e_{i}, i=1,2,3$. A deformation showing general dislocation densities satisfies

$$
-\operatorname{Curl} F=\Lambda_{\mathcal{L}_{1}}^{T}+\Lambda_{\mathcal{L}_{2}}^{T}+\Lambda_{\mathcal{L}_{3}}^{T}=e_{1} \otimes \mathcal{L}_{1}+e_{2} \otimes \mathcal{L}_{2}+e_{3} \otimes \mathcal{L}_{3}
$$

We then assume that

$(\star)$ The graph $\mathcal{G}_{u+v}$ is an integral current and its boundary writes as

$$
\partial \mathcal{G}_{u+v} \equiv \mathcal{L}_{1} \otimes e_{1}+\mathcal{L}_{2} \otimes e_{2}+\mathcal{L}_{3} \otimes e_{3},
$$

(see Section 4). Let us again emphasize that property $(\star)$ holds true under some specific geometric assumptions but at the present stage we do not know if it also holds in the general case. The class of admissible deformation fields reads

$$
\begin{aligned}
\mathcal{F}^{\star}=\left\{F \in L^{p}\left(\hat{\Omega}, \mathbb{R}^{3 \times 3}\right): \operatorname{cof} F \in L^{q}\left(\hat{\Omega} ; \mathbb{R}^{3 \times 3}\right), \operatorname{det} F \in L^{s}(\hat{\Omega}), \operatorname{Div} F \in L^{r}\left(\hat{\Omega} ; \mathbb{R}^{3}\right),\right. \\
\quad-\operatorname{Curl} F=\sum_{i=1}^{3} \Lambda_{\mathcal{L}_{i}}^{T} \text { for integral 1-currents } \mathcal{L}_{i}, F=\hat{F} \text { on } \hat{\Omega} \backslash \Omega, \\
\quad \text { and } F \text { satisfies }(\star)\} .
\end{aligned}
$$

Then, our second existence result is the following (see Theorem 5.3 in Section 5).

Theorem 1.3 (For general Burgers vectors). Let $1<p<2, q>1, s>1$, and $r>\frac{12}{7}$. Under suitable hypotheses on the coefficients (see Theorem 5.3) there exists a minimizer $F \in \mathcal{F}^{\star}$ of $\mathcal{W}$.

Notice that once condition $(\star)$ is satisfied, then we can also drop the hypothesis $q \geq 2$. We conjecture that condition $(\star)$ holds true whenever $q \geq 2$.

\section{The main result: characterization of the graph boundary}

In order to prove the two aforementioned existence results, as said, we need to characterize the boundary of the displacement field $u$ seen as a map with values in the three-dimensional torus. Hence, the major part of the paper is devoted to this effort, yielding the following theorem that we believe relevant as a stand-alone result.

Theorem 1.4 (Characterization of the boundary, cf. Theorem 4.11). Let $u \in S B V\left(\Omega ; \mathbb{R}^{3}\right) \cap$ $W^{1, p}\left(\Omega ; \mathbb{T}^{3}\right)$ be the harmonic map given in Theorem 3.4 below, satisfying (3.12) with $b \in 2 \pi \mathbb{Z}^{3}$. Let $v \in W^{2, r}\left(\Omega ; \mathbb{R}^{3}\right)$, if

$$
r>\frac{12}{7}, \quad p<2, \quad \text { and } \quad \frac{6-2 r}{3 r}+\frac{1}{p} \leq 1,
$$


then the current given by the graph of $u+v$ with values in $\mathbb{T}^{3}$, namely $\mathcal{G}_{u+v} \in \mathcal{D}_{3}\left(\Omega \times \mathbb{T}^{3}\right)$, is a rectifiable integer-multiplicity current with finite mass whose boundary is given by the formula

$$
\partial \mathcal{G}_{u+v}(\omega)=\frac{1}{2 \pi} \int_{0}^{2 \pi} \int_{S_{t}}\left\langle d \omega\left(x, \frac{b \theta}{2 \pi}+v(x)\right),\left(s_{1}, \frac{\partial v}{\partial s_{1}}(x)\right) \wedge\left(s_{2}, \frac{\partial v}{\partial s_{2}}(x)\right) \wedge \vec{b}\right\rangle d \mathcal{H}^{2}(x) d \theta
$$

for all $\omega \in \mathcal{D}^{2}\left(\Omega \times \mathbb{T}^{3}\right)$ and a.e. $t \in[0,2 \pi)$. Here $S_{t}$ is the level set $w=t$ of the map defined by $\frac{b}{2 \pi} w=u$.

Moreover, if $F=\nabla u+\nabla v$ satisfies cof $F \in L^{2}\left(\Omega ; \mathbb{R}^{3 \times 3}\right)$, then the graph $\mathcal{G}_{u+v}$ is integral and its boundary is a 2-dimensional integral current given by

$$
\partial \mathcal{G}_{u+v}(\omega)=\mathcal{L} \wedge b(\omega)=\frac{1}{2 \pi} \int_{0}^{2 \pi} \int_{S_{t}}\left\langle d \omega\left(x, \frac{b \theta}{2 \pi}+v(x)\right),\left(s_{1}, 0\right) \wedge\left(s_{2}, 0\right) \wedge \vec{b}\right\rangle d \mathcal{H}^{2}(x) d \theta
$$

for all $\omega \in \mathcal{D}^{2}\left(\Omega \times \mathbb{T}^{3}\right)$ and a.e. $t \in[0,2 \pi)$.

See Lemma 4.8 and Theorem 4.11 for the detailed statement and notation. We remark that as soon as the field $v$ is sufficiently regular, the condition cof $F \in L^{q}$ with $q \geq 2$ implies that the tangential derivative of $v$ along the dislocation line must vanish (that is, equivalently, that (1.4) holds true). This condition is crucial, since it applies on cof $F$ and not on $v$ which therefore needs not be $\mathcal{C}^{1}$-regular as was the case in [33] (see Theorems 4.1 and 4.2). Specifically, a condition on cof $F$ is physically easier to check, since it is directly related to the growth condition of the bulk energy. For this reason the relaxed hypothesis $r>\frac{12}{7}$ and cof $F \in L^{2}$ in place of $r>3$ is called "physical".

\section{Model variables}

The model deformation variables being $C$ and $D F$ (through the observable $\Gamma$ ) we have to consider their invariants (under suitable maps), since it is natural to assume that the energy must depend only on them. More precisely, the metric tensor $C:=F^{T} F$ is known to be invariant under Euclidean transformations, namely it remains unchanged after superimposing a rigid body motion upon the original motion. Moreover, also the compatibility connexion is such an invariant [8], since it writes in terms of $C$ as

$$
\Gamma=C^{-1} D^{S} C, \quad\left(\left(D^{S} C\right)_{i j k}=\frac{1}{2}\left(\partial_{k} C_{i j}+\partial_{j} C_{i k}-\partial_{i} C_{k j}\right)\right) .
$$

In order to comply with material frame-invariance, for an isotropic body the response laws are given in terms of the invariants of $C$, namely

$$
\begin{aligned}
& \mathbb{I}_{1}(C)=\operatorname{tr} C=F \cdot F, \\
& \mathbb{I}_{2}(C)=\operatorname{tr} \operatorname{cof} C=\operatorname{cof} F \cdot \operatorname{cof} F, \\
& \mathbb{I}_{3}(C)=\operatorname{det} C=(\operatorname{det} F)^{2} .
\end{aligned}
$$

Since in the presence of dislocations we have seen that $F$ never belongs to $L^{2}\left(\Omega ; \mathbb{R}^{3 \times 3}\right)$, the energy has a less than linear growth with respect to the first invariant. As for $\mathbb{I}_{2}(C)$, the cofactor of $F$ is defined as

$$
(\operatorname{cof} F)_{i j}=(F \otimes F)_{i j}:=\frac{1}{2} \epsilon_{i k m} \epsilon_{j l n} F_{k l} F_{m n} .
$$


and the relation $\operatorname{tr} \operatorname{cof} C=\operatorname{cof} F \cdot \operatorname{cof} F$ entails that $\operatorname{tr} \operatorname{cof} C \in L^{1}(\Omega)$ if $\operatorname{cof} F \in$ $L^{2}\left(\Omega ; \mathbb{R}^{3 \times 3}\right)$. This means that, heuristically, the natural hypothesis that $\operatorname{tr} \operatorname{cof} C$ is a summable function requires that $\operatorname{cof} F$ is at least a square-integrable function. Moreover, by the identity

$$
\delta_{i j} \operatorname{det} F=F_{k i}(\operatorname{cof} F)_{k j} .
$$

it follows that, since $F \in L^{p}\left(\Omega ; \mathbb{R}^{3 \times 3}\right)$ with $p<2$ the control of cof $F \in L^{q}\left(\Omega ; \mathbb{R}^{3 \times 3}\right)$ with $q>2$ such that $\frac{1}{p}+\frac{1}{q} \leq 1$ yields a control of $\operatorname{det} F \in L^{1}(\Omega)$. The hypothesis that the energy has a more than linear growth with respect to the second invariant will be crucial in order to get a control on the determinant and to finally prove existence of solutions to (1.3).

Let us also observe that it is a classical requirement to assume $\sqrt{\mathbb{I}_{3}(C)}$ as summable, a condition on the summability of $\operatorname{det} F$. We will see that in the minimization problem, this condition will not play a crucial role, and one could easily consider the incompressible case as well.

As for the higher-order model variable, the only linear invariant of $D F$ is, according to $[5$, Eq.(2.1)],

$$
\mathbb{I}^{\operatorname{lin}}(D F)=\epsilon_{i j k}(D F)_{j k}^{i}=\epsilon_{i j k} F_{m}^{i} \Gamma_{j k}^{m}=\operatorname{tr} \operatorname{Curl} F,
$$

while the third quadratic invariant of $D F$, out of eleven independent invariants [5, Eq.(2.3)], is

$$
\mathbb{I}_{3}^{\text {quad }}(D F)=\delta_{i m} \delta_{j k} \delta_{p q}(D F)_{j k}^{i}(D F)_{p q}^{m}=\operatorname{Div} F \cdot \operatorname{Div} F .
$$

Note that in terms of the invariants $C$ and $\Gamma$ one can write $\mathbb{I}_{3}^{\text {quad }}(D F)=C \cdot \operatorname{tr} \Gamma \otimes \operatorname{tr} \Gamma$.

In the case of gradient hyper-elasticity with dislocations, we will consider energies of the form

$$
\mathcal{W}(F, D F)=\mathcal{W}_{\mathrm{ISO}}(\mathbb{I}(C), \mathbb{I}(D F))=\mathcal{W}_{\mathrm{ISO}}^{1}(\mathbb{I}(C))+\mathcal{W}_{\mathrm{ISO}}^{2}(\mathbb{I}(D F)),
$$

where $\mathbb{I}(C)$ and $\mathbb{I}(D F)$ are the invariants of $C$ and $D F$. The energy $\mathcal{W}_{\mathrm{ISO}}^{2}(\mathbb{I}(D F))$ contains a term

$$
\mathcal{W}_{\text {dislo }}(\operatorname{Curl} F) \text {, }
$$

accounting for anisotropic energy contributions due to the presence of dislocation loops in the otherwise perfect crystal.

We remark that the gradient of $F$ plays a role through two independent terms, the deformation part Div $F$ and the defect part Curl $F$. It is also noticeable that such a higher-order term Div $F$ is sometimes considered as a regularization term (as, e.g., in [19]) to first-grade elasticity. Here we regard it simply as the third quadratic invariant of $D F$.

Let us discuss the assumptions made on the stored elastic energy. For the sake of discussion $F$ is here given the interpretation of the deformation tensor from a reference to the current configuration. Let $v_{i}$ be the principal stretches of $F$, i.e., the eigenvalues of $\sqrt{C}$. A homogeneous and incompressible Ogden material possesses a stored-energy function of the form

$$
W_{\mathrm{OG}}(F)=\frac{c_{1}}{2} \sum_{i=1}^{3}\left(v_{i}^{\alpha}-3\right)+c_{2} \sum_{i \neq j=1}^{3}\left(\left(v_{i} v_{j}\right)^{\beta}-3\right),
$$

with $\alpha, \beta \geq 1$ (see [7]). For $\alpha=\beta=2$ this corresponds to the Mooney-Rivlin material $W_{\mathrm{MR}}=\frac{c_{1}}{2}\left(\mathbb{I}_{1}(C)-3\right)+c_{2}\left(\mathbb{I}_{2}(C)-3\right)$, originally designed for rubber-like materials. Nevertheless Ogden-like energies are also considered for dislocations in finite elasticity (see [42] 
for practical examples). For $\alpha \geq 2$ it is known that $\sum_{i=1}^{3} v_{i}^{\alpha}$ is not in $L^{1}(\Omega)$, in the presence of dislocations (the unsuitability of Mooney materials to describe dislocations was already reported in [42], since it creates the "paradox on longitudinal force").

Therefore, in our model we consider an Ogden material with $\alpha=p<2$, with the identity $|F|^{p}=(F \cdot F)^{p / 2}=(\operatorname{tr} C)^{p / 2}=\left(\sum_{i=1}^{3} v_{i}^{2}\right)^{p / 2} \leq c \sum_{i=1}^{3} v_{i}^{p}$ for some $c>0$, simply meaning that $F \in L^{p}\left(\Omega ; \mathbb{R}^{3 \times 3}\right)$ as soon as the energy is bounded. As for the second term one needs $\sum_{i \neq j=1}^{3}\left(v_{i} v_{j}\right)^{\beta} \in L^{1}(\Omega)$ and this is achieved if $\operatorname{cof} F \cdot \operatorname{cof} F=\operatorname{tr} \operatorname{cof} C=$ $\mathbb{I}_{2}(C) \in L^{1}(\Omega)$ and $\beta \geq 2$. Therefore, we are led to the assumption

$$
\operatorname{cof} F \in L^{q}\left(\Omega ; \mathbb{R}^{3 \times 3}\right),
$$

with $q \geq 2$. This assumption will replace the more artificial one $r>3$.

It is classically known since Rivlin and Saunders' work [29] that for incompressible rubber-like materials the bulk energy $W$ satisfies $\left|\partial_{\mathbb{I}_{2}} W\right|<<\left|\partial_{\mathbb{I}_{1}} W\right|$, i.e., the material response is essentially independent of $\mathbb{I}_{2}$. However, for crystals, to the knowledge of the authors there is no reason to make this assumption. For a compressible material, the classical approach is to add to the bulk energy the term $f(\operatorname{det} F)$ with $f>0$ convex such that $f(t) \rightarrow \infty$ as $t \rightarrow 0^{+}$and satisfying $f(t) \geq c|t|^{s}$ for some $c>0$ and $s>1$. Since $f(\operatorname{det} F)$ must be in $L^{1}(\Omega)$, one requires that

$$
\operatorname{det} F \in L^{s}(\Omega)
$$

with $s>1$. An example of stored elastic energy $\mathcal{W}_{\text {ISO }}^{2}(\mathbb{I}(C))$ used for dislocations in a nonlinear context that shows the simultaneous presence of $\mathbb{I}_{2}$ and $\mathbb{I}_{3}$ is the Blatz-Ko material (see [42, Eq. (3.1.17)]).

As for the term $\mathcal{W}_{\text {ISO }}^{2}(\mathbb{I}(D F))$, it is nowadays a classical approach to consider gradient models (for instance the pioneer work in this respect was [35]) to avoid instabilities in continua submitted to severe loadings and large deformations (see also [19]).

\section{Mathematical formalism}

One of the crucial point is that the very nature of the displacement field in the presence of dislocations is multiple-valued, due to the fact that the value of the displacement field depends on the number of loops made by a circuit wrapping around the dislocation line and along which the deformation is integrated (consider the classical Michell-Cesaro formulae [6]). There are two ways to mathematically address this fact. First, the traditional approach consists in avoiding any such multiple circuits by "closing" the dislocation loop $L$ with a surface $S$ enclosed by $L$. Hence we avoid multivaluedness, but deal with a jump of the displacement on the surface, where it is of constant amplitude. The second approach is to define the displacement as a map with values in the three-dimensional torus. Then, the displacement does not "see" the jumps when loops are made around $L$. We consider these two approaches in [33]. Note that, in addition to their mathematical reason to be, the surface $S$ may be given a physical meaning. Therefore explicit expression of the terms supported by $S$ were also provided in [33]. 


\subsection{Generalities about currents and graphs}

\section{Basic facts}

Let $\alpha$ be a multi-index, i.e., an ordered (increasing) subset of $\{1,2, \ldots, n\}$. We denote by $|\alpha|$ the cardinality (or length) of $\alpha$, and we denote by $\bar{\alpha}$ the complementary set of $\alpha$, i.e., the multi-index given by the ordered set $\{1,2, \ldots, n\} \backslash \alpha$.

For all integers $n>0$ and $k \geq 0$ with $k \leq n$, we denote by $\Lambda_{k} \mathbb{R}^{n}$ the space of $k$-vectors and by $\Lambda^{k} \mathbb{R}^{n}$ the space of $k$-covectors. We denote by $\mathcal{D}^{k}(\Omega)$ the space of smooth and compactly supported $k$-forms, which is a topological vector space. Any $k$-form $\omega \in \mathcal{D}^{k}(\Omega)$ can be written in terms of its components, namely

$$
\omega=\sum_{|\alpha|=k} \varphi_{\alpha} d x_{\alpha},
$$

with $\varphi_{\alpha} \in \mathcal{C}_{c}^{\infty}(\Omega)$, and $d x_{\alpha}$ denoting the $k$-covector, $k=|\alpha|, d x_{\alpha}=d x_{\alpha_{1}} \wedge \cdots \wedge d x_{\alpha_{k}}$. The external derivative of a form $\omega \in \mathcal{D}^{k}(\Omega), k<n$, is the form $d \omega \in \mathcal{D}^{k+1}(\Omega)$ given by $d \omega=\sum_{i=1}^{n} \sum_{|\alpha|=k} \frac{\partial \varphi_{\alpha}}{\partial x_{i}} d x_{i} \wedge d x_{\alpha}$, where $\omega$ has the form (3.1). Given a function $F: U \subset$ $\mathbb{R}^{m} \rightarrow V \subset \mathbb{R}^{n}$ of class $\mathcal{C}^{1}$ and a $k$-form $\omega \in \mathcal{D}^{k}(V)$ the pull-back of $\omega$ by $F$ is the form $F^{\sharp} \omega \in \mathcal{D}^{k}(U)$ defined as

$$
\left\langle F^{\sharp} \omega, v_{1} \wedge \cdots \wedge v_{k}\right\rangle=\left\langle\omega, \frac{\partial F}{\partial v_{1}} \wedge \cdots \wedge \frac{\partial F}{\partial v_{k}}\right\rangle,
$$

for any $k$-vector $v \in \Lambda_{k} \mathbb{R}^{m}$.

\section{The cofactor form}

For any real $n \times n$ matrix $A$ and for $\alpha$ and $\beta$ multi-indices with $|\alpha|+|\beta|=n, M_{\bar{\alpha}}^{\beta}(A)$ denotes the determinant of the submatrix of $A$ obtained by erasing the $i$-th columns and the $j$-th rows, for all $i \in \alpha$ and $j \in \bar{\beta}$. The symbol $M(A)$ denotes the $n$-vector in $\Lambda_{n} \mathbb{R}^{2 n}$ given by

$$
M(A):=\sum_{|\alpha|+|\beta|=n} \sigma(\alpha, \bar{\alpha}) M_{\bar{\alpha}}^{\beta}(A) e_{\alpha} \wedge \varepsilon_{\beta},
$$

where $\left\{e_{i}, \varepsilon_{i}\right\}_{i \leq n}$ is the standard Euclidean basis of $\mathbb{R}^{2 n}$, and $\sigma(\alpha, \bar{\alpha})$ is the sign of the permutation $(\alpha, \bar{\alpha}) \in S(n)$. Accordingly, we define

$$
|M(A)|:=\left(1+\sum_{\substack{|\alpha|+|\beta|=n \\|\beta|>0}}\left|M_{\bar{\alpha}}^{\beta}(A)\right|^{2}\right)^{1 / 2} .
$$

For a matrix $A \in \mathbb{R}^{3 \times 3}$, the symbols adj $A$ and $\operatorname{det} A$ denote the adjugate, i.e., the transpose of the matrix of the cofactors of $A$, and the determinant of $A$, respectively. Explicitly, $M_{j}^{i}(A)=A_{i j}, M_{\bar{j}}^{\bar{i}}(A)=(\operatorname{cof} A)_{i j}=(\operatorname{adj} A)_{j i}, M_{\{1,2,3\}}^{\{1,2,3\}}(A)=\operatorname{det} A$, for $i, j=1,2,3$. Moreover, $|M(A)|=\left(1+\sum_{i, j} A_{i j}^{2}+\sum_{i, j} \operatorname{cof}(A)_{i j}^{2}+\operatorname{det}(A)^{2}\right)^{1 / 2}$.

\section{Currents}

Let $\Omega \subset \mathbb{R}^{n}$ be an open set. The dual space of $\mathcal{D}^{k}(\Omega)$, denoted by $\mathcal{D}_{k}(\Omega)$, is called space of $k$-currents in $\Omega$. We will usually denote currents with capital italic letters. A weak 
convergence in $\mathcal{D}_{k}(\Omega)$ is defined as follows: we say that $\mathcal{T}_{h} \rightarrow \mathcal{T}$ in the sense of currents if for every $\omega \in \mathcal{D}^{k}(\Omega)$ we have $\mathcal{T}_{h}(\omega) \rightarrow \mathcal{T}(\omega)$. The boundary of a current $\mathcal{T} \in \mathcal{D}_{k}(\Omega)$ is the current $\partial \mathcal{T} \in \mathcal{D}_{k-1}(\Omega)$ defined as

$$
\partial \mathcal{T}(\omega)=\mathcal{T}(d \omega), \quad \text { for all } \omega \in \mathcal{D}^{k-1}(\Omega) .
$$

A closed current is a current with null boundary (as, by definition, every current in $\mathcal{D}_{0}(\Omega)$ ). The mass of $\mathcal{T} \in \mathcal{D}_{k}(\Omega)$ is the number $M(\mathcal{T}) \in[0,+\infty]$ given by $M(\mathcal{T}):=$

sup $\mathcal{T}(\omega)$. If $M(\mathcal{T})<+\infty$ then $\mathcal{T}$ turns out to be a Borel measure in $\mathcal{M}_{b}\left(\Omega, \Lambda_{k} \mathbb{R}^{n}\right)$, $\omega \in \mathcal{D}^{k}(\Omega),|\omega| \leq 1$

and its total variation coincides with $M(\mathcal{T})$. It is easily seen that the mass is lower semicontinuous with respect to the weak topology in $\mathcal{D}_{k}(\Omega)$. It is also convenient to introduce the quantity $N(\mathcal{T}):=M(\mathcal{T})+M(\partial \mathcal{T})$, for every $\mathcal{T} \in \mathcal{D}_{k}(\Omega)$.

Let $U \subset \mathbb{R}^{n}$ and $V \subset \mathbb{R}^{m}$ be open sets and $F: U \rightarrow V$ be a smooth map. Then the push-forward of a current $\mathcal{T} \in \mathcal{D}_{k}(U)$ by $F$ is defined as $F_{\sharp} \mathcal{T}(\omega):=\mathcal{T}\left(\zeta F^{\sharp} \omega\right)$ for $\omega \in$ $\mathcal{D}^{k}(V)$, where $F^{\sharp} \omega$ is the standard pull-back of $\omega$ and $\zeta$ is any $\mathcal{C}^{\infty}$-function that is equal to 1 on $\operatorname{spt} \mathcal{T} \cap \operatorname{spt} F^{\sharp} \omega$.

\section{Integral currents}

Let $0 \leq k \leq n$ and let $S \subset \mathbb{R}^{n}$ be a $\mathcal{H}^{k}$-rectifiable set with approximate tangent space $T_{x} S$. If $\tau: S \rightarrow \Lambda_{k}\left(\mathbb{R}^{n}\right)$ and $\theta: S \rightarrow \mathbb{R}$ are $\mathcal{H}^{k}$-integrable functions with $\tau(x) \in T_{x} S$ a simple unit $k$-vector for $\mathcal{H}^{k}$-a.e. $x \in S$, then we can define the current $\mathcal{T}$ as

$$
\mathcal{T}(\omega)=\int_{S}\langle\omega(x), \tau(x)\rangle \theta(x) d \mathcal{H}^{k}(x) \quad \text { for } \omega \in \mathcal{D}^{k}(\Omega) .
$$

Every current for which there exists such $S, \tau$, and $\theta$ is said to be rectifiable. If also its boundary $\partial \mathcal{T}$ is rectifiable, then we adopt the following notation

$$
\mathcal{T} \equiv\{S, \tau, \theta\} .
$$

The current $\mathcal{T} \in \mathcal{D}_{k}(\Omega)$ is rectifiable with integer multiplicity if it is rectifiable, has rectifiable boundary, and the function $\theta$ in (3.3) is integer-valued. An integer-multiplicity current $\mathcal{T}$ such that $N(\mathcal{T})<\infty$ is said an integral current. As known, the simplest example of $n$-dimensional integral current is the integration over a set of finite perimeter $U \subset \mathbb{R}^{n}$. This is denoted by $\llbracket U \rrbracket \in \mathcal{D}_{n}\left(\mathbb{R}^{n}\right)$ and is defined as

$$
\llbracket U \rrbracket(\omega)=\int_{U}\langle\omega(x), \tau\rangle d x \quad \text { for all } \omega \in \mathcal{D}^{n}\left(\mathbb{R}^{n}\right),
$$

where $\tau:=e_{1} \wedge \cdots \wedge e_{n}$ the standard orientating vector of $\mathbb{R}^{n}$.

If $S=F(U)$ where $F: U \subset \mathbb{R}^{n} \rightarrow \mathbb{R}^{m}$ is a map smooth enough, we can define the push forward by $F$ of the current $\llbracket U \rrbracket$, which writes as $F_{\sharp} \llbracket U \rrbracket(\omega)=\llbracket U \rrbracket\left(F^{\sharp} \omega\right)=\int_{U}\left\langle F^{\sharp} \omega, \tau\right\rangle d x=$ $\int_{S}\left\langle\omega, \frac{\partial F}{\partial x_{1}} \wedge \cdots \wedge \frac{\partial F}{\partial x_{n}}\right\rangle d \mathcal{H}^{n}$.

An integral current $\mathcal{T} \in \mathcal{D}_{M}\left(\mathbb{R}^{n}\right)$ is said indecomposable if there exists no integral current $\mathcal{R}$ such that $\mathcal{R} \neq 0 \neq \mathcal{T}-\mathcal{R}$ and

$$
N(\mathcal{T})=N(\mathcal{R})+N(\mathcal{T}-\mathcal{R}) .
$$

The following theorem provides a decomposition property of every integral current and the structure characterization of integer-multiplicity indecomposable 1-currents (see [17, Section 4.2.25]). 
Theorem 3.1 (Federer). For every integral current $\mathcal{T}$ there exists a sequence of indecomposable integral currents $\mathcal{T}_{i}$ such that

$$
\mathcal{T}=\sum_{i} \mathcal{T}_{i} \quad \text { and } \quad N(\mathcal{T})=\sum_{i} N\left(\mathcal{T}_{i}\right)
$$

Suppose $\mathcal{T}$ is an indecomposable integer multiplicity 1-current on $\mathbb{R}^{n}$. Then there exists a Lipschitz function $f: \mathbb{R} \rightarrow \mathbb{R}^{n}$ with $\operatorname{Lip}(f) \leq 1$ such that

$$
f\left\llcorner[0, M(\mathcal{T})] \text { is injective and } \mathcal{T}=f_{\sharp}[[0, M(\mathcal{T})] \rrbracket \text {. }\right.
$$

Moreover $\partial \mathcal{T}=0$ if and only if $f(0)=f(M(\mathcal{T}))$.

In other words, an indecomposable integral 1-current turns out to be a simple Lipschitz curve.

\section{Graph currents}

Let us consider the space $\Omega \times \mathbb{R}^{3}$. We will use the Euclidean coordinates $x=\left(x_{1}, x_{2}, x_{3}\right)$ for $x \in \Omega$ and $y=\left(y_{1}, y_{2}, y_{3}\right)$ for $y \in \mathbb{R}^{3}$. Every 3 -form $\omega \in \mathcal{D}^{3}\left(\Omega \times \mathbb{R}^{3}\right)$ can be decomposed as $\omega=\sum \omega_{\alpha \beta} d x^{\alpha} \wedge d y^{\beta}$, with $\omega_{\alpha \beta} \in \mathcal{C}_{c}^{\infty}\left(\Omega \times \mathbb{R}^{3}\right)$, where the sum is computed over all multi-indices $\alpha$ and $\beta$ such that $|\alpha|+|\beta|=3$.

Let $p>1$. We denote by $\mathcal{A}_{p}\left(\Omega, \mathbb{R}^{3}\right)$ the space:

$$
\begin{aligned}
& \mathcal{A}_{p}\left(\Omega, \mathbb{R}^{3}\right):=\left\{u \in L^{1}\left(\Omega ; \mathbb{R}^{3}\right): u \text { is approx. differentiable a.e. on } \Omega,\right. \text { and } \\
&\left.M_{\bar{\alpha}}^{\beta}(D u) \in L^{p}(\Omega) \text { for all }|\alpha|+|\beta|=3\right\},
\end{aligned}
$$

The symbol $M_{\bar{\alpha}}^{\beta}(D u)$ has been introduced in (3.2) with $A=D u$. If $u \in \mathcal{A}_{p}\left(\Omega, \mathbb{R}^{3}\right)$ then $\mathcal{G}_{u}$, the current carried by the graph of $u$, is defined as follows:

$$
\mathcal{G}_{u}:=(I d \times u)_{\sharp} \llbracket \Omega \rrbracket,
$$

where $I d \times u: \mathbb{R}^{3} \rightarrow \mathbb{R}^{3} \times \mathbb{R}^{3}$ is given by $(I d \times u)(x)=(x, u(x))$, viz.,

$$
\mathcal{G}_{u}(\omega)=\int_{\Omega} \sigma(\alpha, \bar{\alpha}) \omega_{\alpha \beta}(x, u(x)) M_{\bar{\alpha}}^{\beta}(D u(x)) d x,
$$

for all $\omega=\omega_{\alpha \beta} d x^{\alpha} \wedge d y^{\beta} \in \mathcal{D}^{3}\left(\Omega \times \mathbb{R}^{3}\right)$. Moreover if $u \in \mathcal{A}_{p}\left(\Omega, \mathbb{R}^{3}\right)$ then $\mathcal{G}_{u}$ turns out to be a integer-multiplicity 3 -current in $\Omega \times \mathbb{R}^{3}$.

\subsection{Graphs of maps with values in $\mathbb{T}^{3}$}

The flat torus $\mathbb{T}$ is defined as $\mathbb{T} \cong \mathbb{R} / \sim$, with $\sim$ the equivalent relation given by

$$
a \sim b \text { if and only if } a-b \in 2 \pi \mathbb{Z} .
$$

In [33] (see also [32]) we studied the graphs of maps $u: \Omega \rightarrow \mathbb{T}^{3}$ seen as currents in $\mathcal{D}_{3}\left(\Omega \times \mathbb{T}^{3}\right)$. This space can be seen as the dual of the topological vector space $\mathcal{D}^{3}\left(\Omega \times \mathbb{T}^{3}\right)$, consisting of all compactly supported smooth 3 -forms in $\Omega \times \mathbb{T}^{3}$. By (3.7) it can be easily deduced that $\mathcal{D}^{3}\left(\Omega \times \mathbb{T}^{3}\right)$ coincides with the class of smooth 3 -forms in $\Omega \times \mathbb{R}^{3}$ which are $2 \pi$-periodic in the last three variables. As a consequence, there is a natural embedding 
from the space of currents in $\mathcal{D}_{3}\left(\Omega \times \mathbb{R}^{3}\right)$ with compact support in $\bar{\Omega} \times \mathbb{R}^{3}$ to $\mathcal{D}^{3}\left(\Omega \times \mathbb{T}^{3}\right)$, obtained by restricting $\mathcal{T} \in \mathcal{D}_{3}\left(\Omega \times \mathbb{R}^{3}\right)$ to the space of 3 -forms in $\Omega \times \mathbb{R}^{3}$ which are $2 \pi$-periodic in the last three variables. More precisely, if $\mathcal{T} \in \mathcal{D}_{3}\left(\Omega \times \mathbb{R}^{3}\right)$ has compact support in $\bar{\Omega} \times \mathbb{R}^{3}$ then the following current $T(\mathcal{T}) \in \mathcal{D}_{3}\left(\Omega \times \mathbb{T}^{3}\right)$ is well defined as

$$
T(\mathcal{T}):=\mathcal{T}\left\llcorner\mathcal{D}^{3}\left(\Omega \times \mathbb{T}^{3}\right),\right.
$$

where $\mathcal{D}^{3}\left(\Omega \times \mathbb{T}^{3}\right)$ denotes the class of 3 -forms in $\Omega \times \mathbb{R}^{3}$ which are $2 \pi$-periodic in the last three variables. Since in general smooth functions in $\Omega \times \mathbb{R}^{3}$ are not periodic in the last three variables, it is easily seen that $M(T(\mathcal{T})) \leq M(\mathcal{T})$.

The procedure of embedding a current in $\mathcal{D}_{3}\left(\Omega \times \mathbb{R}^{3}\right)$ into $\mathcal{D}_{3}\left(\Omega \times \mathbb{T}^{3}\right)$ can be applied to graphs of functions $u \in \mathcal{A}_{p}\left(\Omega, \mathbb{R}^{3}\right)$; we define $T(u): \Omega \rightarrow \mathbb{T}^{3}$ using the projection $\pi_{T}: \mathbb{R} \rightarrow \mathbb{T}$, namely $T\left(u_{i}\right):=\pi_{T}\left(u_{i}\right)$ for $i=1,2,3$. Since $\pi_{T}$ is a smooth functions, $\mathcal{G}_{T(u)}$ turns out to be 3 -rectifiable current in $\Omega \times \mathbb{T}^{3}$ whenever $\mathcal{G}_{u}$ is a 3 -rectifiable current in $\Omega \times \mathbb{R}^{3}$. Furthermore it holds $\mathcal{G}_{T(u)}=T\left(\mathcal{G}_{u}\right)$.

We introduce the space $\mathcal{A}_{p}\left(\Omega, \mathbb{T}^{3}\right)$ as follows:

$$
\begin{aligned}
& \mathcal{A}_{p}\left(\Omega, \mathbb{T}^{3}\right):=\left\{u \in L^{1}\left(\Omega ; \mathbb{T}^{3}\right): u \text { is approx. differentiable a.e. on } \Omega,\right. \text { and } \\
&\left.M_{\bar{\alpha}}^{\beta}(D u) \in L^{p}(\Omega) \text { for all }|\alpha|+|\beta|=3\right\},
\end{aligned}
$$

where $L^{1}\left(\Omega ; \mathbb{T}^{3}\right)$ coincides with the space of measurable functions $u: \Omega \rightarrow \mathbb{T}^{3}$.

A weak convergence is defined on $\mathcal{A}_{p}\left(\Omega, \mathbb{T}^{3}\right)$. Namely, we say that a sequence $u_{k} \in$ $\mathcal{A}_{p}\left(\Omega, \mathbb{T}^{3}\right)$ weakly converges to $u \in \mathcal{A}_{p}\left(\Omega, \mathbb{T}^{3}\right)$ if $u_{k} \rightarrow u$ strongly in $L^{1}\left(\Omega ; \mathbb{T}^{3}\right)$, and $M_{\bar{\alpha}}^{\beta}\left(D u_{k}\right) \rightarrow M_{\bar{\alpha}}^{\beta}\left(D u_{k}\right)$ weakly in $L^{p}(\Omega)$ for all multi-indices $|\alpha|+|\beta|=3$ (see [21, Section 3.3.3]).

Following the classical theory of Cartesian currents (see, [20], [21]), it is straightforward that if $u \in \mathcal{A}_{p}\left(\Omega, \mathbb{T}^{3}\right)$ then the graph $\mathcal{G}_{u}$ is a integer multiplicity 3-current. Moreover, since the mass of a current does not increase by composition with $T$, if there exists $\bar{u} \in \mathcal{A}_{p}\left(\Omega, \mathbb{R}^{3}\right)$ such that $T(\bar{u})=u$ and $\mathcal{G}_{\bar{u}}$ is an integral current, then $\mathcal{G}_{u}$ is an integral current.

Lemma 3.2. Let $u_{k}, u \in \mathcal{A}_{p}\left(\Omega, \mathbb{T}^{3}\right)$ be such that $u_{k} \rightarrow u$ weakly in $\mathcal{A}_{p}\left(\Omega, \mathbb{T}^{3}\right)$, then $\mathcal{G}_{u_{k}} \rightarrow \mathcal{G}_{u}$ as currents. $3.3 .2]$.

The following closure Theorem is a standard adaptation of Theorem 2 of [21, Section

Theorem 3.3 (Federer-Fleming). Let $u_{k}$ be a sequence in $\mathcal{A}_{p}\left(\Omega, \mathbb{T}^{3}\right)$ such that $u_{k} \rightarrow$ $u$ strongly in $L^{p}\left(\Omega ; \mathbb{T}^{3}\right)$ and suppose that there exist functions $v_{\bar{\alpha}}^{\beta} \in L^{p}(\Omega)$ such that $M_{\bar{\alpha}}^{\beta}\left(D u_{k}\right) \rightarrow v_{\bar{\alpha}}^{\beta}$ weakly for all multi-indices $\alpha$ and $\beta$ with $|\alpha|+|\beta|=3$. If

$$
M\left(\partial \mathcal{G}_{u_{k}}\right)<C<+\infty
$$

for all $k>0$, then $u \in \mathcal{A}_{p}\left(\Omega, \mathbb{T}^{3}\right)$ and $v_{\alpha}^{\beta}=M_{\bar{\alpha}}^{\beta}(D u)$.

Let us remark that the original proof of the closure result on which the last theorem is based is due to Federer and Fleming, but that was only established for currents in Euclidean spaces. The theory for the flat torus can be obtained as adaptation of the more general theory for $G$ chains developed first by Fleming, and then by White, De Pauw and Hardt (see for instance $[14,15]$ and references therein). 


\subsection{The displacement and the dislocation-induced deformation: basic results}

Let us start with a technical result. Let $\hat{\Omega}$ be a bounded open set with smooth boundary, let $g \in \mathcal{C}^{1, \alpha}\left(\partial \hat{\Omega} ; \mathbb{R}^{3}\right)$ be such that $\int_{\partial \hat{\Omega}} g d \mathcal{H}^{2}=0$, and let $v \in \mathcal{C}^{2, \alpha}\left(\hat{\Omega} ; \mathbb{R}^{3}\right)$ be the zeroaverage-value solution to

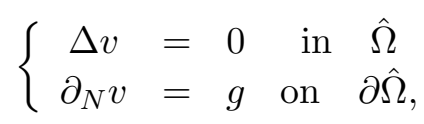

then $\|v\|_{\mathcal{C}^{2, \alpha}} \leq C\|g\|_{\mathcal{C}^{1, \alpha}}$, with $C=C(\hat{\Omega})$ (see [22, Theorem 6.30 and Theorem 6.31]).

Theorem 3.4 (Harmonic map [37]). Let L be a Lipschitz closed curve in $\mathbb{R}^{3}$ and $S$ a bounded Lipschitz surface with boundary $L$ and unit normal $N$. Let $b \in \mathbb{R}^{3}$. The solution of the system

$$
\begin{cases}\Delta u=0 & \text { in } \quad \mathbb{R}^{3} \backslash S \\ {[u]:=u^{+}-u^{-}=b} & \text { on } S \\ {\left[\partial_{N} u\right]:=\partial_{N} u^{+}-\partial_{N} u^{-}=0} & \text { on } S\end{cases}
$$

is given by (up to a harmonic map in $\mathbb{R}^{3}$ )

$$
u_{i}(x)=-b_{i} \int_{S} \partial_{N} \Phi\left(x^{\prime}-x\right) d \mathcal{H}^{2}\left(x^{\prime}\right),
$$

for $x \in \mathbb{R}^{3} \backslash S$, where $\Phi$ is the the fundamental solution of the Laplacian in $\mathbb{R}^{3}$, namely $\Delta \Phi=\delta_{0}$.

In the following theorem the symbol $B V^{p}$ denotes the space of functions with bounded variation whose absolutely continuous part of the gradient belongs to $L^{p}, p \geq 1$.

Theorem 3.5 (Nature of the displacement field [33]). Let L be a closed Lipschitz curve in $\Omega$ and let $b \in 2 \pi \mathbb{Z}^{3}$. Then for any Lipschitz surface $S$ with boundary $L$, every solution $u$ to (3.11) belongs to $B V^{p}\left(\Omega ; \mathbb{R}^{3}\right)$ with $p=\frac{3}{2}$, satisfies $\operatorname{Div} \nabla^{a} u=0$ and $-\operatorname{Curl} \nabla^{a} u=b \otimes \mathcal{L}$ as distributions (here $\nabla^{a} u$ is the part of the gradient of $u$ that is absolutely continuous with respect to the Lebesgue measure). Moreover if the curve $L$ is of class $\mathcal{C}^{2}$, then $\nabla^{a} u \in$ $L^{p}\left(\Omega ; \mathbb{R}^{3 \times 3}\right)$ for all $1 \leq p<2$. As a consequence, $u$ can be seen with values in $\mathbb{T}^{3}$, in which case it happens that $T(u) \in W^{1, p}\left(\Omega ; \mathbb{T}^{3}\right)$, and $\nabla T(u)=\nabla^{a} u$. Furthermore, $T(u)$ does not depend on the specific surface $S$ enclosing $L$, but only depends on $L$.

We will often consider $u$ as torus-valued, thus dropping the symbol $T$ and denoting $T(u)$ by $u$ as well. As a consequence, in the sequel the identification

$$
\nabla u \equiv \nabla^{a} u
$$

will mean that the gradient of the torus-valued map $u$ equals to the absolutely continuous part of the distributional derivative of the vector-valued $S B V$-map $u$.

Remark 3.6. Let us emphasize that the integral in (3.12) (and then the content of Theorem 3.5) can be extended, by Federer decomposition Theorem 3.1, to any integer multiplicity 2-current $S \subset \mathbb{R}^{3}$. Indeed, if $\mathcal{L}$ is an integral 1-current, it can be decomposed 
as sum of simple Lipschitz curves $\mathcal{L}_{i}$, each of which having a surface $S_{i}$ enclosing it. Then formula (3.12) can be generalized as the sum of the same integral on all $S_{i}$. It is easy to see that the absolutely continuous part of the gradient of the resulting $u$ does not depend on the choice of the surfaces $S_{i}$.

If $S$ has boundary $\mathcal{L}$ an integral 1-current, then it follows that

$$
-\operatorname{Curl} \nabla u=b \otimes \mathcal{L}
$$

This will be used in our main result (Theorem 4.11).

Theorem 3.7 (Biot-Savart $[9,33])$. Let $\hat{\Omega} \subset \mathbb{R}^{3}$ be an open simply connected and smooth set. Let $\mu$ be a tensor-valued Radon measure such that $\mu \in \mathcal{M}_{\text {div }}\left(\hat{\Omega} ; \mathbb{R}^{3 \times 3}\right.$ ) (where the label div stands for divergence-free). Then there exists a unique $F \in L^{1}\left(\hat{\Omega} ; \mathbb{R}^{3 \times 3}\right)$ solution of

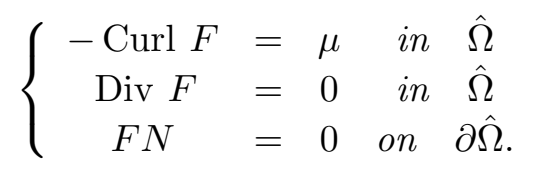

Moreover $F$ belongs to $L^{p}\left(\hat{\Omega} ; \mathbb{R}^{3 \times 3}\right)$ for all $p$ with $1 \leq p \leq 3 / 2$ and for all such $p$ there exists a constant $C>0$ satisfying

$$
\|F\|_{p} \leq C|\mu|(\hat{\Omega}) .
$$

In the case that $\mu=b \otimes \tau \mathcal{H}^{1}\left\llcorner\mathcal{L}\right.$, for some $b \in \mathbb{R}^{3}$ and a $\mathcal{C}^{2}$-closed curve $\mathcal{L}$ in $\hat{\Omega}$ with unit oriented tangent vector $\tau$, then the solution $F$ belongs to $L^{p}\left(\hat{\Omega} ; \mathbb{R}^{3 \times 3}\right)$ for all $p<2$.

Note that improved regularity for $F$ applies, as discussed in Section 5.4.

\section{An archetypal result}

Let $\mathcal{L}$ be a closed loop (or in general a closed integral 1-current) and let $\eta$ be a field defined on $\Omega$. Let $u_{i}$ be the map introduced in (3.12) with $b_{i}=2 \pi$, and consider it as torus-valued, namely $u_{i} \in W^{1, p}(\Omega ; \mathbb{T})$. To prove several of the following results we will use the following coarea formula

$$
\int_{0}^{2 \pi} \int_{\left\{u_{i}=t\right\}} \eta(x) d \mathcal{H}^{2}(x) d t=\int_{\Omega} \eta(x)\left|\nabla u_{i}(x)\right| d x .
$$

Now, if $\int_{\left\{u_{i}=t\right\}} \eta(x) d \mathcal{H}^{2}(x)$ is proved to be independent of $t \in[0,2 \pi)$, then one also has the following identity:

$$
\int_{\left\{u_{i}=t\right\}} \eta(x) d \mathcal{H}^{2}(x)=\frac{1}{2 \pi} \int_{\Omega} \eta(x)\left|\nabla u_{i}(x)\right| d x .
$$

The following Lemma is proved by means of this formula.

Lemma 3.8. Let $\mathcal{L} \in \mathcal{D}_{1}(\Omega)$ be an integral closed 1-current supported in $\Omega$, and let $w \in W^{1,3 / 2}(\Omega ; \mathbb{T})$ be the harmonic map given by formula $(3.12)$ with $b_{i}=2 \pi$. Let $S_{t}:=$ $\{x \in \Omega: w(x)=t\}$. Then for a.e. $t \in[0,2 \pi)$ the surface $S_{t}$ is the support of an integral 2 -current $\mathcal{S}_{t}$ in $\mathcal{D}_{2}(\Omega)$ such that $\partial \mathcal{S}_{t}=\mathcal{L}$. 
Proof. Let $L$ be the support set of the 1-current $\mathcal{L}$. Let $\varphi \in \mathcal{C}_{c}^{\infty}\left(\Omega ; \mathbb{R}^{3}\right)$ be arbitrary, we have

$$
\begin{aligned}
& \int_{L} \varphi \cdot \tau d \mathcal{H}^{1}=-\int_{\Omega} \operatorname{Curl} \varphi \cdot \nabla w d x=-\int_{\Omega} \operatorname{Curl} \varphi \cdot \frac{\nabla w}{|\nabla w|}|\nabla w| d x \\
& =-\int_{0}^{2 \pi} \int_{S_{t}} \operatorname{Curl} \varphi \cdot N_{t} d \mathcal{H}^{2} d t=\int_{0}^{2 \pi} \int_{\partial S_{t}} \varphi \cdot \tau_{t} d \mathcal{H}^{1} d t
\end{aligned}
$$

where $N_{t}=\frac{\nabla w}{|\nabla w|}$ is the unit normal to $S_{t}$, and in the last equality we have employed the Stokes Theorem. By arbitrariness of $\varphi$ we deduce that the distribution given by $\varphi \mapsto \int_{0}^{2 \pi} \int_{\partial S_{t}} \varphi \cdot \tau_{t} d \mathcal{H}^{1} d t$ coincides with $\tau \mathcal{H}^{1}\left\llcorner_{L}\right.$, which implies the thesis.

As a consequence of the preceding lemma, and adopting the notation in (3.4), it follows that for a.e. $t \in[0,2 \pi)$ the integer multiplicity current

$$
\mathcal{S}_{t}=\left\{S_{t}, \tau=N_{t}, \theta=1\right\},
$$

is integral with boundary $\partial \mathcal{S}_{t}=\mathcal{L}$. In particular the multiplicity of $\mathcal{S}_{t}$ is 1 .

\subsection{Preliminaries on dislocations at the continuum scale}

We call a dislocation loop any simple closed curve $\mathcal{L}$ in $\Omega$ which has an associated Burgers vector $b \in 2 \pi \mathbb{Z}^{3}$. We say that a $\mathbb{R}^{3 \times 3}$-valued field $F$ is a deformation in the presence of dislocation $\mathcal{L}$ and Burgers vector $b$ if it satisfies the condition

$$
-\operatorname{Curl} F=\Lambda_{\mathcal{L}^{b}}^{T}:=b \otimes \tau \mathcal{H}^{1}\llcorner\mathcal{L},
$$

where $\tau$ is an oriented tangent vector to $\mathcal{L}$ defined $\mathcal{H}^{1}$ almost everywhere. Given any $b \in 2 \pi \mathbb{Z}^{3}$ we call a $b$-dislocation current a closed integral 1 -current $\mathcal{L}^{b}$ with associated Burgers vector $b$, and its corresponding density, denoted by $\Lambda_{\mathcal{L}^{b}} \in \mathcal{M}_{b}\left(\Omega, \mathbb{R}^{3 \times 3}\right)$, is the divergence-free measure satisfying

$$
\left\langle\Lambda_{\mathcal{L}^{b}}, w\right\rangle=\mathcal{L}^{b}\left((w b)^{*}\right),
$$

for every $w \in \mathcal{C}_{c}^{\infty}\left(\Omega, \mathbb{R}^{3 \times 3}\right)$, where in the right-hand side $(w b)^{*}$ is the covector writing $(w b)^{*}:=w_{k j} b_{j} d x_{k}$ (with Einstein convention on repeated indices). We will employ the following notation:

$$
\Lambda_{\mathcal{L}^{b}}=\mathcal{L}^{b} \otimes b=\tau^{b} \otimes b \theta^{b} \mathcal{H}^{1}\llcorner\mathcal{L},
$$

with $\theta^{b}$ the multiplicity of the vector $b$ (see [32] for details). Notice that the dislocation density can be identified with the dislocation current if seen as integral currents with coefficients in $\mathbb{Z}^{3}$; we however prefer to use the label "dislocation density" when we treat it as a Radon measure.

Definition 3.9 (Regular dislocation). We denote by $\mathcal{B}:=2 \pi \mathbb{Z}^{3}$ the lattice of admissible Burgers vectors. A regular dislocation is a sequence of $b$-dislocation currents $\mathcal{L}:=\left\{\mathcal{L}^{b}\right\}_{b \in \mathcal{B}}$. To each dislocation is associated a dislocation current, still denoted by $\mathcal{L}:=\sum_{b \in \mathcal{B}} \mathcal{L}^{b}$, and the associated dislocation density $\Lambda_{\mathcal{L}}:=\sum_{b \in \mathcal{B}} \Lambda_{\mathcal{L}^{b}}$.

Given a regular dislocation $\mathcal{L}$ it is possible to split it on the canonical basis of $\mathbb{R}^{3}$, $\mathcal{L}=\mathcal{L}_{1}+\mathcal{L}_{2}+\mathcal{L}_{3}$, in such a way that $\mathcal{L}_{i}$ has $2 \pi e_{i}$ as associated Burgers vector and satisfies

$$
\Lambda_{\mathcal{L}}=\Lambda_{\mathcal{L}_{1}}+\Lambda_{\mathcal{L}_{2}}+\Lambda_{\mathcal{L}_{3}}=2 \pi \sum_{i=1}^{3} \mathcal{L}_{i} \otimes e_{i}
$$


Moreover, as proved in [32], one has $\left|\mathcal{L}_{i}\right|_{\Omega} \leq c\left|\Lambda_{\mathcal{L}}\right|_{\mathcal{M}_{b}(\Omega)}$, for some constant $c>0$ independent of $i$ and $\Omega$.

A tensor $F \in L^{p}\left(\Omega ; \mathbb{R}^{3 \times 3}\right)$, with $p>1$, is called a deformation field in the presence of the regular dislocation $\mathcal{L}$ if it satisfies

$$
-\operatorname{Curl} F=\Lambda_{\mathcal{L}}^{T}=\Lambda_{\mathcal{L}_{1}}^{T}+\Lambda_{\mathcal{L}_{2}}^{T}+\Lambda_{\mathcal{L}_{3}}^{T}=2 \pi \sum_{i=1}^{3} e_{i} \otimes \mathcal{L}_{i} .
$$

\subsection{Properties of the dislocation-induced deformation}

By Helmholtz decomposition we can write any deformation field $F \in L^{p}\left(\Omega ; \mathbb{R}^{3 \times 3}\right)$ in the presence of the regular dislocation $\mathcal{L}$ as the sum of a compatible and a incompatible part

$$
F=\nabla \tilde{v}+F^{0}
$$

where $F^{0}$ is the unique solution of

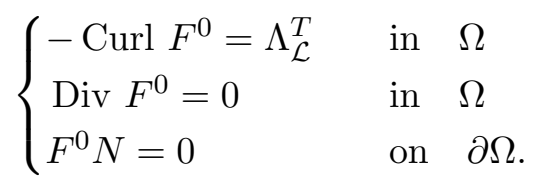

By Theorem 3.7 and Lemmas 3.4 and 3.5, it is easy to see that the incompatible field $F^{0}$ is, up to a harmonic map, given by the sum of three fields $\nabla^{a} u_{i}, i=1,2,3$, where $u_{i}$ is the map given by (3.12) with $b=2 \pi e_{i}$ and $S$ being the support of an arbitrary integral 2-current $\mathcal{S} \in \mathcal{D}_{2}(\Omega)$ with boundary $\partial \mathcal{S}=\mathcal{L}_{i}, i=1,2,3$. Namely, there exists a smooth harmonic map $u_{0} \in \mathcal{C}^{\infty}\left(\bar{\Omega} ; \mathbb{T}^{3}\right)$ such that

$$
F^{0}=\nabla u_{0}+\sum_{i=1}^{3} \nabla u_{i}
$$

with $u_{i} \in W^{1,3 / 2}\left(\Omega ; \mathbb{T}^{3}\right)$ given by (3.12) with $b=2 \pi e_{i}, i=1,2,3$. Moreover, if $F^{0} \in$ $L^{p}\left(\Omega ; \mathbb{R}^{3 \times 3}\right)$ with $p \in(3 / 2,2)$, then, since $u_{0}$ is regular on $\Omega$, we infer $u_{i} \in W^{1, p}\left(\Omega ; \mathbb{T}^{3}\right)$ for $i=1,2,3$. The compatible part of $F$, we have that $\tilde{v}$ in $(3.22)$ satisfies $-\Delta \tilde{v}=-\operatorname{Div} F$, so that if $-\operatorname{Div} F \in L^{r}\left(\Omega ; \mathbb{R}^{3}\right)$ then $\tilde{v} \in W^{2, r}\left(\Omega ; \mathbb{R}^{3}\right)$. As for $u_{0}$, this is harmonic and smooth. We denote by $v:=\tilde{v}+u_{0}$.

In summary, we can always decompose a deformation field $F \in L^{p}\left(\Omega ; \mathbb{R}^{3 \times 3}\right)$ as sum of three gradients

$$
F=\nabla \tilde{v}+\nabla u_{0}+\nabla u=\nabla v+\nabla u
$$

where the function $v=\tilde{v}+u_{0}$ has values in $\mathbb{R}^{3}$ and $u$ in $\mathbb{T}^{3}$. Furthermore, by standard projection $v$ can be in turn identified with a torus-valued map. Starting from this key representation, we can consider the graph $\mathcal{G}_{u+v}$ of the map $v+u \in W^{1, p}\left(\Omega ; \mathbb{T}^{3}\right)$ as an integer multiplicity 3-current in the space $\Omega \times \mathbb{T}^{3}$, and, up to characterize its boundary $\partial \mathcal{G}_{u+v}$, it is possible to adapt standard closure results for Cartesian currents to our case, thus getting compactness principles for minimizing sequences of the problem (1.3). 
It is worth here to emphasize the strict connection between this representation and the fact that the Burgers vectors are constrained to stay in the lattice $\mathcal{B}$. Actually, in $[32,33]$ it was proved that whenever a field $F \in L^{p}\left(\Omega ; \mathbb{R}^{3 \times 3}\right)$ satisfies $(3.21)$ then it can be decomposed as in (3.25). The fact that $u$ and $v$ can be seen as maps with values in the flat torus is a direct consequence of equation (3.21), and such correspondence can be obtained by the procedure described in Section 3.2. This procedure only consists in identifying the displacement modulo a vector in $2 \pi \mathbb{Z}^{3}$; in turn, this identification consists, when we look at $u$ and $v$, in restricting their graph currents to the space of 3 -forms which are $2 \pi$-periodic in the second variable. A complete theory of closedness for this class of currents is then provided by the corresponding results for $G$ chains $[14,15]$.

We stress out that the domain $\Omega \times \mathbb{T}^{3}$ for the graphs associated to $u$ and $v$ is not a convenient choice but is required by the choice of the lattice $\mathcal{B}=2 \pi \mathbb{Z}^{3}$, since, as already said, $u$ and $v$ have values in $\mathbb{T}^{3}$ as a consequence of (3.21). A different choice of the lattice $\mathcal{B}$ would give rise to a different target space for $u$ and $v$.

Let us now focus on the mechanical induced deformation $v$. A control of the $L^{r}$ norm of Div $F$ provides us the control of the $W^{2, r}$-norm of $v$. Indeed, Div $F=\Delta v \in$ $L^{r}\left(\Omega ; \mathbb{R}^{3}\right)$, so that $v \in W^{2, r}\left(\Omega ; \mathbb{R}^{3}\right), \nabla v \in W^{1, r}\left(\Omega ; \mathbb{R}^{3 \times 3}\right)$ and $\nabla^{2} v \in L^{r}\left(\Omega ; \mathbb{R}^{3 \times 3 \times 3}\right)$. By Sobolev embedding, $\nabla v \in L^{s}\left(\Omega ; \mathbb{R}^{3 \times 3}\right)$ with $s=\frac{3 r}{3-r}$. Now, one has by $(2.4)$ that ( Curl cof $\nabla v)_{i j}=\epsilon_{i l k}\left(v_{l, j} v_{k, n n}+v_{l, j n} v_{k, n}\right)$, and hence by Hölder inequality Curl cof $\nabla v \in$ $L^{t}\left(\Omega ; \mathbb{R}^{3 \times 3}\right)$ with $t=\frac{3 r}{6-r}$. Thus Curl Curl cof $\nabla v=-\Delta \operatorname{cof} \nabla v \in W^{-1, t}\left(\Omega ; \mathbb{R}^{3 \times 3}\right)$ (by the identity Div cof $\nabla v=0)$ and hence $\operatorname{cof} \nabla v \in W^{1, t}\left(\Omega ; \mathbb{R}^{3 \times 3}\right)$, that is, once more by Sobolev embedding, cof $\nabla v \in L^{h}\left(\Omega ; \mathbb{R}^{3 \times 3}\right)$ with $h=\frac{3 r}{6-2 r}$. By $(2.5)$ we have $I$ det $\nabla v=$ $\nabla^{T} v$ cof $\nabla v$, hence by Hölder again $\operatorname{det} \nabla v \in L^{m}(\Omega)$ with $m=\frac{r}{3-r}$. Thus we state the following result:

Lemma 3.10. Let $\Omega \subset \mathbb{R}^{3}$ be a bounded Lipschitz open set. Let $v \in W^{2, r}\left(\Omega ; \mathbb{R}^{3}\right)$, then cof $\nabla v \in W^{1, t}\left(\Omega ; \mathbb{R}^{3 \times 3}\right)$ with $t=\frac{3 r}{6-r}$.

Owing once again to the decomposition (3.25), and assuming that the dislocationinduced deformation $\nabla u$ is generated by a single Burgers vector $b$ (that, without loss of generality, we assume $b=e_{1}$ ), the term $\operatorname{det} F$ can be written as the sum

$$
\operatorname{det} F=\operatorname{det}(\nabla v+\nabla u)=\operatorname{det}\left(\begin{array}{c}
\nabla v_{1} \\
\nabla v_{2} \\
\nabla v_{3}
\end{array}\right)+\operatorname{det}\left(\begin{array}{c}
\nabla u_{1} \\
\nabla v_{2} \\
\nabla v_{3}
\end{array}\right) .
$$

Hence, since det $\nabla v \in L^{\frac{r}{3-r}}(\Omega)$ and since cof $\nabla v \in L^{\frac{3 r}{6-2 r}}\left(\Omega ; \mathbb{R}^{3 \times 3}\right)$, in order that $\operatorname{det} F \in$ $L^{1}(\Omega)$ it suffices, again by Hölder, that $\frac{6-2 r}{3 r}+\frac{1}{p} \leq 1$, that is

$$
r \geq \frac{6 p}{5 p-3} .
$$

Since $p<2$ this entails the natural requirement $r>\frac{12}{7}$. Note that $r$ may still be less than 2 .

In particular the preceding discussion yields the following Lemma:

Lemma 3.11. Let $F \in L^{p}\left(\Omega ; \mathbb{R}^{3 \times 3}\right)$ be such that (3.25) holds true with $v \in W^{2, r}\left(\Omega ; \mathbb{R}^{3}\right)$ and let $u \in W^{1, p}\left(\Omega ; \mathbb{T}^{3}\right)$ be the solution of $(3.11)$ with $b=e_{1}$. Suppose $p<2$ and $r>\frac{12}{7}$ be such that (3.27) holds true. Then $\operatorname{det} F \in L^{s}(\Omega)$ with

$$
\frac{1}{s}=\frac{6-2 r}{3 r}+\frac{1}{p} \text {. }
$$




\section{Boundary of graphs}

\subsection{Preliminary results}

Let $L$ be a Lipschitz closed and simple curve, let $b \in 2 \pi \mathbb{Z}^{3}$ be a fixed Burgers vector, and let $v \in \mathcal{C}^{1}\left(\Omega ; \mathbb{R}^{3}\right)$. We introduce the currents $\mathcal{L} \wedge b$ and $\mathcal{C}_{u+v}$ belonging to $\mathcal{D}_{2}\left(\Omega \times \mathbb{T}^{3}\right)$ and defined as follows:

$$
\begin{aligned}
& \mathcal{L} \wedge b(\omega)=\frac{1}{2 \pi} \int_{0}^{2 \pi} \int_{L}\left\langle\omega\left(x, \frac{b \theta}{2 \pi}+v(x)\right), \vec{\tau}(x) \wedge \vec{b}\right\rangle d \mathcal{H}^{1}(x) d \theta \\
& \mathcal{C}_{u+v}(\omega)=\frac{1}{2 \pi} \int_{0}^{2 \pi} \int_{L}\left\langle\omega\left(x, \frac{b \theta}{2 \pi}+v(x)\right), \frac{\partial \vec{v}}{\partial \tau}(x) \wedge \vec{b}\right\rangle d \mathcal{H}^{1}(x) d \theta,
\end{aligned}
$$

for all $\omega \in \mathcal{D}^{2}\left(\Omega \times \mathbb{T}^{3}\right)$. Here $\tau(x) \in \mathbb{R}^{3}$ is the tangent vector to $L$ at the point $x$, $\vec{\tau}:=(\tau, 0) \in \Lambda_{1} \mathbb{R}^{6}, \vec{b}:=(0, b) \in \Lambda_{1} \mathbb{R}^{6}, \vec{v}=(0, v) \in \Lambda_{1} \mathbb{R}^{6}$. From this point on we will use the arrow to distiguish $b$ (and similarly the other vectors), which belongs to $\mathbb{R}^{3}$, from the 1-vector $\vec{b}:=(0, b) \in \Lambda_{1} \mathbb{R}^{6}$.

The following result [33, Theorem 4.1] shows that the boundary of the graph of the torus-valued displacement field $u$ is related to the dislocation density.

Theorem 4.1 (Dislocation density as a graph boundary [33]). Let $S$ be a simple Lipschitz surface in $\Omega$ whose boundary is $L$, a simple Lipschitz and closed curve in $\Omega$. Let $b=$ $\left(b_{1}, b_{2}, b_{3}\right) \in 2 \pi \mathbb{Z}^{3}$, let $u=\left(u_{1}, u_{2}, u_{3}\right): \Omega \rightarrow \mathbb{R}^{3}$ be the map given by (3.12), and let $v=0$. Then $u \in W^{1, \frac{3}{2}}\left(\Omega ; \mathbb{T}^{3}\right)$ and $\mathcal{G}_{u}$ is an integral current in $\mathcal{D}_{3}\left(\Omega \times \mathbb{T}^{3}\right)$ whose boundary is given by

$$
\partial \mathcal{G}_{u}(\omega)=\mathcal{L} \wedge b(\omega),
$$

for all $\omega \in \mathcal{D}^{2}\left(\Omega \times \mathbb{T}^{3}\right)$. In particular $\left|\partial \mathcal{G}_{u}\right|=|\mathcal{L} \wedge b|=|\mathcal{L} \otimes b|$.

The following result [33, Theorem 4.6.] gives a representation of the boundary of the graph of a torus-valued displacement field $u$ when it is perturbed by a regular displacement $v$ (i.e., associated to the compatible deformation $\nabla v$ ).

Theorem 4.2. Let $S, L, b$ and $u$ be as in Theorem 4.1, and let $v \in \mathcal{C}^{1}\left(\bar{\Omega}, \mathbb{R}^{3}\right)$. Then $\mathcal{G}_{u+v}$ is the integral current in $\mathcal{D}_{3}\left(\Omega \times \mathbb{T}^{3}\right)$ given by

$$
\partial \mathcal{G}_{u+v}(\omega)=\mathcal{L} \wedge b(\omega)+\mathcal{C}_{u+v}(\omega),
$$

for all $\omega \in \mathcal{D}^{2}\left(\Omega \times \mathbb{T}^{3}\right)$, with $\mathcal{C}_{u+v}$ defined in (4.1). In particular, it holds

$$
M\left(\partial \mathcal{G}_{u+v}\right) \leq C\left(1+\|D v\|_{L^{\infty}}\right)|\mathcal{L} \otimes b|(\Omega),
$$

for some general constant $C>0$.

\subsection{Weak form of the graph boundaries}

The aim here is to express the graph boundaries in (4.3) as integrals over a suitable current $\mathcal{S}$ with boundary $\mathcal{L}$ (by Stokes Theorem, or equivalently, by definition of boundary for currents). This will provide a weak formulation of (4.1) valid for less regular fields $v$. 
Introducing the map $\Phi: \Omega \times[0,2 \pi] \rightarrow \Omega \times \mathbb{R}^{3}$ given by $\Phi(x, \theta)=\left(x, \frac{b \theta}{2 \pi}+v(x)\right)$, it is easy to see that the current $\mathcal{J}_{v}:=\mathcal{L} \wedge b+\mathcal{C}_{u+v}$ can be written as

$$
\begin{aligned}
\mathcal{J}_{v}(\omega) & =\int_{0}^{2 \pi} \int_{L}\left\langle\Phi^{\sharp} \omega, \vec{\tau} \wedge \vec{t}\right\rangle d \mathcal{H}^{1}(x) d \theta=\llbracket L \times[0,2 \pi] \rrbracket\left(\Phi^{\sharp} \omega\right) \\
& =\Phi_{\sharp} \llbracket L \times[0,2 \pi] \rrbracket(\omega), \quad \forall \omega \in \mathcal{D}_{2}\left(\Omega \times \mathbb{T}^{3}\right),
\end{aligned}
$$

where $\vec{t}$ is the tangent vector to the segment $[0,2 \pi) \subset \Omega \times[0,2 \pi)$ (and with abuse of notation now $\vec{\tau}$ is the tangent vector to $L \times[0,2 \pi) \subset \Omega \times[0,2 \pi)$, hence $\left.\vec{\tau}, \vec{t} \in \Lambda_{1} \mathbb{R}^{4}\right)$. Using the fact that the form $\omega$ is $2 \pi$-periodic in the second variable, this can be rewritten by definition of boundary as

$$
\begin{aligned}
\mathcal{J}_{v}(\omega) & =\partial\left(\Phi_{\sharp} \llbracket S \times[0,2 \pi] \rrbracket\right)(\omega)=\Phi_{\sharp} \llbracket S \times[0,2 \pi] \rrbracket(d \omega) \\
& =\frac{1}{2 \pi} \int_{0}^{2 \pi} \int_{S}\left\langle d \omega\left(x, \frac{b \theta}{2 \pi}+v(x)\right),\left(s_{1}, \partial_{s_{1}} v(x)\right) \wedge\left(s_{2}, \partial_{s_{2}} v(x)\right) \wedge \vec{b}\right\rangle d \mathcal{H}^{2}(x) d \theta,
\end{aligned}
$$

where $S$ is a Lipschitz simple surface with boundary $L$ and with orienting simple vector $s_{1} \wedge s_{2}, s_{1}, s_{2} \in T_{S}$ and with $\partial_{a} v:=\nabla v \cdot a$ for a vector $a \in \mathbb{R}^{3}$.

Explicitly, we can decompose $\mathcal{J}_{v}$ with respect to a (local) orthonormal coordinate system: let us denote $s_{3}=s_{1} \times s_{2}$ so that $\left\{s_{1}, s_{2}, s_{3}\right\}$ is an orthonormal basis of $\mathbb{R}^{3}$ with associated coordinates $\left\{\xi_{1}, \xi_{2}, \xi_{3}\right\}$. Since $s_{3}=N$ the unit normal to the surface $S$, it is evident that this system of coordinates is local; we will still denote by $\left\{x_{1}, x_{2}, x_{3}\right\}$ the standard coordinates system associated to the Euclidean basis $\left\{e_{1}, e_{2}, e_{3}\right\}$. The nonconstant change-of-basis matrix will be denoted by $A \in \mathbb{R}^{3 \times 3}$, so that a vector $a \in \mathbb{R}^{3}$ has coordinates $a_{i}^{N}:=A_{i j} a_{j}$ with respect to the basis $\left\{s_{1}, s_{2}, s_{3}\right\}$. The matrix $A_{i j}=A_{i j}(x)$ depends on $x \in S \subset \Omega$, and since it is unitary, its $L^{\infty}$-norm is bounded in $\Omega$, namely

$$
A_{i j} \in L^{\infty}\left(\Omega ; \mathbb{R}^{3 \times 3}\right) .
$$

Let $\left\{b /|b|, b_{1}^{\perp}, b_{2}^{\perp}\right\}$ be an orthonormal basis of $\mathbb{R}^{3}$ with $\left\{y_{1}, y_{2}, y_{3}\right\}$ as associated Cartesian system. Let us first consider the $i 1$-component of $\mathcal{J}_{v}$, for $i \in\{1,2,3\}$. This, recalling that $d x_{i}=A_{i k} d \xi_{k}$, is

$$
\begin{aligned}
\mathcal{J}_{v}\left(\varphi d x_{i} \wedge d y_{1}\right)=\mathcal{J}_{v}^{i, 1}(\varphi)= \\
=\frac{1}{2 \pi} \int_{0}^{2 \pi} \int_{S}\left\langle\frac{\partial \varphi}{\partial x_{j}}\left(x, \frac{b \theta}{2 \pi}+v(x)\right) A_{j k} A_{i l} d \xi_{k} \wedge d \xi_{l} \wedge d y_{1}, N_{v}^{b}\right\rangle d \mathcal{H}^{2}(x) d \theta \\
\quad+\frac{1}{2 \pi} \int_{0}^{2 \pi} \int_{S}\left\langle\frac{\partial \varphi}{\partial y_{j}}\left(x, \frac{b \theta}{2 \pi}+v(x)\right) A_{i l} d \xi_{l} \wedge d y_{1} \wedge d y_{j}, N_{v}^{b}\right\rangle d \mathcal{H}^{2}(x) d \theta
\end{aligned}
$$

where

$$
N_{v}^{b}=\left(s_{1}, \partial_{s_{1}} v(x)\right) \wedge\left(s_{2}, \partial_{s_{2}} v(x)\right) \wedge \vec{b} .
$$

It is now easy to compute

$$
\left\langle d \xi_{k} \wedge d \xi_{l} \wedge d y_{1}, N_{v}^{b}\right\rangle= \begin{cases}|b| & \text { for } k=1, l=2 \\ -|b| & \text { for } l=1, k=2 \\ 0 & \text { otherwise }\end{cases}
$$


while

$$
\left\langle d \xi_{l} \wedge d y_{1} \wedge d y_{j}, N_{v}^{b}\right\rangle= \begin{cases}(-1)^{l}|b| \frac{\partial v_{j}}{\partial \xi_{l c}} & \text { for } l \in\{1,2\}, j \neq 1, \\ 0 & \text { otherwise, }\end{cases}
$$

where, in the last expression, $l^{c}:=\{1,2\} \backslash\{l\}$. Eventually, from (4.7) we have

$$
\begin{aligned}
& \mathcal{J}_{v}\left(\varphi d x_{i} \wedge d y_{1}\right)=\mathcal{J}_{v}^{i, 1}(\varphi)= \\
& \quad \frac{|b|}{2 \pi} \int_{0}^{2 \pi} \int_{S} \frac{\partial \varphi}{\partial x_{j}}\left(x, \frac{b \theta}{2 \pi}+v(x)\right)\left(A_{j 1}(x) A_{i 2}(x)-A_{j 2}(x) A_{i 1}(x)\right) d \mathcal{H}^{2}(x) d \theta \\
& \quad+\frac{|b|}{2 \pi} \int_{0}^{2 \pi} \int_{S} \frac{\partial \varphi}{\partial y_{j}}\left(x, \frac{b \theta}{2 \pi}+v(x)\right)\left(\frac{\partial v_{j}}{\partial \xi_{1}}(x) A_{i 2}(x)-\frac{\partial v_{j}}{\partial \xi_{2}}(x) A_{i 1}(x)\right) d \mathcal{H}^{2}(x) d \theta,
\end{aligned}
$$

A similar computation yields the following expression

$$
\begin{aligned}
& \mathcal{J}_{v}\left(\varphi d y_{1} \wedge d y_{i}\right)=\mathcal{J}_{v}^{0,1 i}(\varphi)= \\
& =\frac{(-1)^{i}|b|}{2 \pi} \int_{0}^{2 \pi} \int_{S} \frac{\partial \varphi}{\partial y_{i} c}\left(x, \frac{b \theta}{2 \pi}+v(x)\right)\left(\frac{\partial v_{2}}{\partial \xi_{1}} \frac{\partial v_{3}}{\partial \xi_{2}}-\frac{\partial v_{3}}{\partial \xi_{1}} \frac{\partial v_{2}}{\partial \xi_{2}}\right)(x) d \mathcal{H}^{2}(x) d \theta \\
& \quad-\frac{|b|}{2 \pi} \sum_{j=1}^{3} \int_{0}^{2 \pi} \int_{S} \frac{\partial \varphi}{\partial x_{j}}\left(x, \frac{b \theta}{2 \pi}+v(x)\right)\left(A_{j 1}(x) \frac{\partial v_{i}}{\partial \xi_{2}}(x)-A_{j 2}(x) \frac{\partial v_{i}}{\partial \xi_{1}}(x)\right) d \mathcal{H}^{2}(x) d \theta
\end{aligned}
$$

valid for $\omega=\varphi d y_{1} \wedge d y_{i}(i=2,3), \varphi \in \mathcal{C}_{c}^{\infty}\left(\Omega \times \mathbb{T}^{3}\right)$. In the expression for $\mathcal{J}_{v}^{0,1 i}, i \in\{2,3\}$, we have denoted by $i^{c}=\{2,3\} \backslash\{i\}$. From (4.1) it is evident that the current $\mathcal{J}_{v}$ is nonzero only if computed on forms of the type $\omega=\varphi d x_{i} \wedge d y_{1}(i=1,2,3)$ or $\omega=\varphi d y_{1} \wedge d y_{i}$ $(i=2,3)$, since all the other components turn out to be identically zero. Namely, we have

$$
\begin{aligned}
& \mathcal{J}_{v}\left(\varphi d x_{i} \wedge d x_{j}\right)=\mathcal{J}_{v}^{i j, 0}(\varphi)=0 \\
& \mathcal{J}_{v}\left(\varphi d y_{2} \wedge d y_{3}\right)=\mathcal{J}_{v}^{0,23}(\varphi)=0 .
\end{aligned}
$$

Moreover the currents $\mathcal{L} \wedge b$ and $\mathcal{C}_{u+v}$ act on different components. More precisely, from (4.1) we infer the following properties:

$$
\begin{aligned}
& \mathcal{L} \wedge b(\omega)=0 \text { if } \omega \neq \varphi d x_{i} \wedge d y_{1} \text { for some } i=1,2,3, \\
& \mathcal{C}_{u+v}(\omega)=0 \text { if } \omega \neq \varphi d y_{1} \wedge d y_{j} \text { for some } j=2,3,
\end{aligned}
$$

which implies that

$$
\begin{aligned}
\mathcal{J}_{v}\left(\varphi d x_{i} \wedge d y_{1}\right) & =\mathcal{L} \wedge b\left(\varphi d x_{i} \wedge d y_{1}\right) \\
\mathcal{J}_{v}\left(\varphi d y_{1} \wedge d y_{j}\right) & =\mathcal{C}_{u+v}\left(\varphi d y_{1} \wedge d y_{j}\right)
\end{aligned}
$$

\subsection{Case of a single dislocation}

\section{Weak expression of the graph boundary}

The following lemma gathers some properties of graph boundary related to a single dislocation loop.

Lemma 4.3. Let $L$ be a simple Lipschitz closed curve in $\Omega$, let $u=\left(u_{1}, u_{2}, u_{3}\right) \in$ $S B V\left(\Omega ; \mathbb{R}^{3}\right) \cap W^{1, p}\left(\Omega ; \mathbb{T}^{3}\right)$ be the harmonic map of Theorem 3.4, satisfying (3.12) with $b \in 2 \pi \mathbb{Z}^{3}$. The following assertions hold true: 
(a) Let $v \in \mathcal{C}^{1}\left(\bar{\Omega} ; \mathbb{R}^{3}\right)$. Then $\mathcal{G}_{u+v}$ is an integral current in $\mathcal{D}_{3}\left(\Omega \times \mathbb{T}^{3}\right)$ whose boundary is given by

$$
\begin{aligned}
\partial \mathcal{G}_{u+v}(\omega) & =\mathcal{J}_{v}(\omega)= \\
= & \frac{1}{2 \pi} \int_{0}^{2 \pi} \int_{S}\left\langle d \omega\left(x, \frac{b \theta}{2 \pi}+v(x)\right),\left(s_{1}, \frac{\partial v}{\partial s_{1}}(x)\right) \wedge\left(s_{2}, \frac{\partial v}{\partial s_{2}}(x)\right) \wedge \vec{b}\right\rangle d \mathcal{H}^{2}(x) d \theta,
\end{aligned}
$$

for all $\omega \in \mathcal{D}^{2}\left(\Omega \times \mathbb{T}^{3}\right)$.

(b) The current $\mathcal{J}_{v}$ does not depend on the Lipschitz surface $S$ in the formula in (a) which encloses L. Moreover the value $\mathcal{J}_{v}(\omega)$ does depend only on the value of $\omega \in$ $\mathcal{D}^{2}\left(\Omega \times \mathbb{T}^{3}\right)$ in a neighborhood of $L$. In particular, if $\omega$ and $\tilde{\omega}$ coincide in such a neighborhood, then $\mathcal{J}_{v}(\omega-\tilde{\omega})=0$.

(c) Let $r>\frac{12}{7}$ and $p<2$ be such that

$$
\frac{6-2 r}{3 r}+\frac{1}{p} \leq 1
$$

and assume $\nabla u \in L^{p}\left(\Omega ; \mathbb{R}^{3 \times 3}\right)$. Let $v \in W^{2, r}\left(\Omega ; \mathbb{R}^{3}\right)$, and let $v_{n} \in \mathcal{C}^{\infty}\left(\bar{\Omega} ; \mathbb{R}^{3}\right)$ be a sequence of functions such that $v_{n} \rightarrow v$ strongly in $W^{2, r}\left(\Omega ; \mathbb{R}^{3}\right)$. Then for all $\omega \in \mathcal{D}^{2}\left(\Omega \times \mathbb{T}^{3}\right)$ we have

$$
\mathcal{J}_{v_{n}}(\omega) \rightarrow \mathcal{J}_{v}(\omega)
$$

where

$$
\begin{aligned}
& \mathcal{J}_{v}(\omega):=\frac{1}{2 \pi} \int_{0}^{2 \pi} \int_{S_{t}}\left\langle d \omega\left(x, \frac{b \theta}{2 \pi}+v(x)\right),\left(s_{1}, \frac{\partial v}{\partial s_{1}}(x)\right) \wedge\left(s_{2}, \frac{\partial v}{\partial s_{2}}(x)\right) \wedge \vec{b}\right\rangle d \mathcal{H}^{2}(x) d \theta \\
& =\frac{1}{(2 \pi)^{2}} \int_{0}^{2 \pi} \int_{\Omega}\left\langle d \omega\left(x, \frac{b \theta}{2 \pi}+v(x)\right),\left(s_{1}, \frac{\partial v}{\partial s_{1}}(x)\right) \wedge\left(s_{2}, \frac{\partial v}{\partial s_{2}}(x)\right) \wedge \vec{b}|\nabla w(x)|\right\rangle d x d \theta
\end{aligned}
$$

where $w$ has values in $\mathbb{T}$ and is defined by $w \frac{b}{2 \pi}:=u$, and $S_{t}:=\{w=t\}$ is an arbitrary level set of $w, t \in[0,2 \pi)$ (notice that the last expression is well defined thanks to the fact that $\nabla v \in W^{1, r}$ has pointwise meaning $\mathcal{H}^{2}$-a.e. on $S_{t}$ since $r>1$ ). In particular, the value of $\mathcal{J}_{v}(\omega)$ does not depend on the specific surface $S_{t}$, namely does not depend on $t \in[0,2 \pi)$, and depends only on the values of $\omega$ in a neighborhood of $L \times \mathbb{T}^{3}$.

Proof. Step 0: Statement (a) is the content of Theorem 4.2. The expression of the boundary is obtained by application of Stokes Theorem as in (4.5) and (4.6), where $S$ is an arbitrary simple Lipschitz surface enclosed by $L$. Thanks to the fact that $\mathcal{J}_{v}=\mathcal{L} \wedge b+\mathcal{C}_{u+v}$ has the expression given in (4.1), also statement (b) follows straightforwardly.

Let us prove assertion (c). To demonstrate (4.12) we will show that any component of $\mathcal{J}_{v_{n}}$ as in (4.8a) converges to the correspondent component of $\mathcal{J}_{v}$. We have to treat the two components in (4.8a) and (4.8b).

Step 1: Convergence of $(4.8 \mathrm{~b})$. We have to prove that

$$
\mathcal{J}_{v_{n}}^{0,12}(\varphi) \rightarrow \mathcal{J}_{v}^{0,12}(\varphi)
$$

for any $\varphi \in \mathcal{C}_{c}^{\infty}\left(\Omega \times \mathbb{R}^{3}\right)$ that is $2 \pi$-periodic in the second variable. First we observe that, thanks to point (b) the value of $\mathcal{J}_{v_{n}}^{0,12}(\varphi)$ does not depend on the specific surface $S$ 
chosen in (4.8b). Thanks to Lemma 3.8 we can take $S=S_{t}$, a level surface of the function $w \in W^{1, p}(\Omega ; \mathbb{T})$, which is defined in such a way that $u=\frac{b}{2 \pi} w$ (note that $u$, by (3.12), is always parallel to $b$ ), namely $S_{t}:=\{x \in \Omega: w(x)=t \in[0,2 \pi)\}$. By (4.8b) it holds

$$
\begin{aligned}
& \mathcal{J}_{v_{n}}^{0,12}(\varphi)=\frac{|b|}{2 \pi}\left(\int_{0}^{2 \pi} \int_{S_{t}} \frac{\partial \varphi}{\partial y_{3}}\left(x, \frac{b \theta}{2 \pi}+v_{n}(x)\right)\left(\frac{\partial\left(v_{n}\right)_{2}}{\partial \xi_{1}} \frac{\partial\left(v_{n}\right)_{3}}{\partial \xi_{2}}-\frac{\partial\left(v_{n}\right)_{3}}{\partial \xi_{1}} \frac{\partial\left(v_{n}\right)_{2}}{\partial \xi_{2}}\right)(x) d \mathcal{H}^{2}(x) d \theta\right. \\
& \left.-\sum_{j=1}^{3} \int_{0}^{2 \pi} \int_{S_{t}} \frac{\partial \varphi}{\partial x_{j}}\left(x, \frac{b \theta}{2 \pi}+v_{n}(x)\right)\left(\frac{\partial\left(v_{n}\right)_{1}}{\partial \xi_{2}}(x) A_{j 1}(x)-\frac{\partial\left(v_{n}\right)_{2}}{\partial \xi_{1}}(x) A_{j 2}(x)\right) d \mathcal{H}^{2}(x) d \theta\right) .
\end{aligned}
$$

Since this expression does not depend on $t \in[0,2 \pi)$, we also have

$$
\begin{aligned}
& \mathcal{J}_{v_{n}}^{0,12}(\varphi)=\frac{1}{2 \pi} \int_{0}^{2 \pi} \mathcal{J}_{v_{n}}^{0,12}(\varphi) d t= \\
& \frac{|b|}{(2 \pi)^{2}}\left(\int_{0}^{2 \pi} \int_{0}^{2 \pi} \int_{S_{t}} \frac{\partial \varphi}{\partial y_{3}}\left(x, \frac{b \theta}{2 \pi}+v_{n}(x)\right)\left(\frac{\partial\left(v_{n}\right)_{2}}{\partial \xi_{1}} \frac{\partial\left(v_{n}\right)_{3}}{\partial \xi_{2}}-\frac{\partial\left(v_{n}\right)_{3}}{\partial \xi_{1}} \frac{\partial\left(v_{n}\right)_{2}}{\partial \xi_{2}}\right)(x) d \mathcal{H}^{2}(x) d \theta d t\right. \\
& \left.-\sum_{j=1}^{3} \int_{0}^{2 \pi} \int_{0}^{2 \pi} \int_{S_{t}} \frac{\partial \varphi}{\partial x_{j}}\left(x, \frac{b \theta}{2 \pi}+v_{n}(x)\right)\left(\frac{\partial\left(v_{n}\right)_{1}}{\partial \xi_{2}}(x) A_{j 1}(x)-\frac{\partial\left(v_{n}\right)_{2}}{\partial \xi_{1}}(x) A_{j 2}(x)\right) d \mathcal{H}^{2}(x) d \theta d t\right) .
\end{aligned}
$$

Let us show the convergence of the first line, which is the most involved (the other two lines are treated similarly, see the following remark). For all $\theta \in[0,2 \pi)$, we observe that the quantity

$$
\int_{0}^{2 \pi} \int_{S_{t}} \frac{\partial \varphi}{\partial y_{3}}\left(x, \frac{b \theta}{2 \pi}+v_{n}(x)\right)\left(\frac{\partial\left(v_{n}\right)_{2}}{\partial \xi_{1}} \frac{\partial\left(v_{n}\right)_{3}}{\partial \xi_{2}}-\frac{\partial\left(v_{n}\right)_{3}}{\partial \xi_{1}} \frac{\partial\left(v_{n}\right)_{2}}{\partial \xi_{2}}\right)(x) d \mathcal{H}^{2}(x) d t .
$$

coincides, by coarea formula, with

$$
\int_{\Omega} \frac{\partial \varphi}{\partial y_{3}}\left(x, \frac{b \theta}{2 \pi}+v_{n}(x)\right)\left(\frac{\partial\left(v_{n}\right)_{2}}{\partial \xi_{1}} \frac{\partial\left(v_{n}\right)_{3}}{\partial \xi_{2}}-\frac{\partial\left(v_{n}\right)_{3}}{\partial \xi_{1}} \frac{\partial\left(v_{n}\right)_{2}}{\partial \xi_{2}}\right)(x)|\nabla w(x)| d x
$$

We now observe that the term $D_{n}:=\left(\frac{\partial\left(v_{n}\right)_{2}}{\partial \xi_{1}} \frac{\partial\left(v_{n}\right)_{3}}{\partial \xi_{2}}-\frac{\partial\left(v_{n}\right)_{3}}{\partial \xi_{1}} \frac{\partial\left(v_{n}\right)_{2}}{\partial \xi_{2}}\right)|\nabla w|$ coincides with

$$
D_{n}=\frac{2 \pi}{|b|} \operatorname{det}\left(\begin{array}{c}
\nabla_{\xi} u_{1} \\
\nabla_{\xi}\left(v_{n}\right)_{2} \\
\nabla_{\xi}\left(v_{n}\right)_{3}
\end{array}\right)
$$

with $\nabla_{\xi}$ standing for the gradient in the local basis. Remark also that det $\left(\begin{array}{c}\nabla_{x} u_{1} \\ \nabla_{x}\left(v_{n}\right)_{2} \\ \nabla_{x}\left(v_{n}\right)_{3}\end{array}\right)=$ $\operatorname{det}\left(\left(\begin{array}{c}\nabla_{\xi} u_{1} \\ \nabla_{\xi}\left(v_{n}\right)_{2} \\ \nabla_{\xi}\left(v_{n}\right)_{3}\end{array}\right) A^{t}\right)=\operatorname{det}\left(\begin{array}{c}\nabla_{\xi} u_{1} \\ \nabla_{\xi}\left(v_{n}\right)_{2} \\ \nabla_{\xi}\left(v_{n}\right)_{3}\end{array}\right)$. Hence, recalling that $u_{1},\left(v_{n}\right)_{2}$ and $\left(v_{n}\right)_{3}$ are expressed in the basis $\left\{b /|b|, b_{1}^{\perp}, b_{2}^{\perp}\right\}$, it follows that the value of $D_{n}$ does not depend on the local basis $\left\{s_{1}, s_{2}, s_{3}\right\}$. To prove (4.18), observe that $P_{n}:=\frac{\partial\left(v_{n}\right)_{2}}{\partial \xi_{1}} \frac{\partial\left(v_{n}\right)_{3}}{\partial \xi_{2}}-\frac{\partial\left(v_{n}\right)_{3}}{\partial \xi_{1}} \frac{\partial\left(v_{n}\right)_{2}}{\partial \xi_{2}}=$ $\left(\operatorname{cof} \nabla v_{n}\right)_{13}=\left(\left(\operatorname{cof} \nabla v_{n}\right) s_{3}\right)_{1}$, where $s_{3}$ corresponds to the vector $N$. Moreover $\nabla u_{1}=$ 
$\frac{|b|}{2 \pi} \nabla w$ and $\nabla w=|\nabla w| N$, so that $D_{n}=\left(\operatorname{cof} \nabla v_{n}\right)_{1} \cdot \nabla w$ from which (4.18) follows. Now, by Lemma 3.10 we have cof $\nabla v_{n} \in W^{1, t}\left(\Omega ; \mathbb{R}^{3 \times 3}\right)$ with $t=\frac{3 r}{6-r}$; by Sobolev embedding cof $\nabla v_{n} \in L^{q}\left(\Omega ; \mathbb{R}^{3 \times 3}\right)$ with $q \leq \frac{3 r}{6-2 r}$, and since $u_{1} \in L^{p}\left(\Omega ; \mathbb{R}^{3}\right)$, by (4.11) we infer that $D_{n} \rightarrow D:=\frac{2 \pi}{|b|} \operatorname{det}\left(\begin{array}{c}\nabla u_{1} \\ \nabla v_{2} \\ \nabla v_{3}\end{array}\right) \quad$ in $L^{1}(\Omega)$. From this we conclude that the quantity in (4.17) converges to

$$
\int_{\Omega} \frac{\partial \varphi}{\partial y_{3}}\left(x, \frac{b \theta}{2 \pi}+v(x)\right)\left(\frac{\partial v_{2}}{\partial \xi_{1}} \frac{\partial v_{3}}{\partial \xi_{2}}-\frac{\partial v_{3}}{\partial \xi_{1}} \frac{\partial v_{2}}{\partial \xi_{2}}\right)(x)|\nabla w(x)| d x,
$$

thanks to the fact that $\frac{\partial \varphi}{\partial y_{3}}$ is of class $\mathcal{C}^{\infty}$ (the component with coordinate $y_{3}$ is not local), and $v_{n}$ converges to $v$ in $L^{\infty}\left(\Omega ; \mathbb{R}^{3}\right)$ (since $r>3 / 2$ ). Moreover, by Hölder inequality, it is easy to see that the quantity in (4.17) is bounded by a constant independent of $\theta \in[0,2 \pi)$. We have hence proved, by dominated convergence Theorem, that the first line in (4.15) converges to

$$
\frac{|b|}{(2 \pi)^{2}} \int_{0}^{2 \pi} \int_{\Omega} \frac{\partial \varphi}{\partial y_{3}}\left(x, \frac{b \theta}{2 \pi}+v(x)\right)\left(\frac{\partial v_{2}}{\partial \xi_{1}} \frac{\partial v_{3}}{\partial \xi_{2}}-\frac{\partial v_{3}}{\partial \xi_{1}} \frac{\partial v_{2}}{\partial \xi_{2}}\right)(x)|\nabla w(x)| d x d \theta
$$

The second line in (4.15) can be proved to converge to

$$
-\frac{|b|}{(2 \pi)^{2}} \sum_{j=1}^{3} \int_{0}^{2 \pi} \int_{\Omega} \frac{\partial \varphi}{\partial x_{j}}\left(x, \frac{b \theta}{2 \pi}+v_{n}(x)\right)\left(\frac{\partial v_{1}}{\partial \xi_{2}}(x) A_{j 1}(x)-\frac{\partial v_{2}}{\partial \xi_{1}}(x) A_{j 2}(x)\right) d x d \theta .
$$

Since the argument is very similar to the previous one, we omit the details here and refer to Remark 4.4 below. Summarizing we have shown that the term in (4.15) converges to the sum of (4.20) and (4.21), that we denote by $\int_{0}^{2 \pi} \int_{\Omega} Q_{\theta}|\nabla w(x)| d x d \theta$. It remains to show that

$$
\int_{0}^{2 \pi} \int_{\Omega} Q_{\theta}|\nabla w(x)| d x d \theta=2 \pi \int_{0}^{2 \pi} \int_{S_{t}} Q_{\theta} d \mathcal{H}^{2}(x) d \theta
$$

for any $t \in[0,2 \pi)$. This will conclude the proof of (4.13) together with the fact that $\mathcal{J}_{v}^{0,12}(\varphi)$ does not depend on $t \in[0,2 \pi)$. We will prove that the first line in (4.14) converges to

$$
\frac{|b|}{2 \pi} \int_{0}^{2 \pi} \int_{S_{t}} \frac{\partial \varphi}{\partial y_{3}}\left(x, \frac{b \theta}{2 \pi}+v(x)\right)\left(\frac{\partial v_{2}}{\partial \xi_{1}} \frac{\partial v_{3}}{\partial \xi_{2}}-\frac{\partial v_{3}}{\partial \xi_{1}} \frac{\partial v_{2}}{\partial \xi_{2}}\right)(x) d \mathcal{H}^{2}(x) d \theta
$$

The argument above provides that we can use the dominated convergence Theorem, so it suffices to show that for all fixed $\theta \in[0,2 \pi)$

$$
\int_{S_{t}} \frac{\partial \varphi}{\partial y_{3}}\left(x, \frac{b \theta}{2 \pi}+v_{n}(x)\right)\left(\frac{\partial\left(v_{n}\right)_{2}}{\partial \xi_{1}} \frac{\partial\left(v_{n}\right)_{3}}{\partial \xi_{2}}-\frac{\partial\left(v_{n}\right)_{3}}{\partial \xi_{1}} \frac{\partial\left(v_{n}\right)_{2}}{\partial \xi_{2}}\right)(x) d \mathcal{H}^{2}(x)
$$

tends to

$$
\int_{S_{t}} \frac{\partial \varphi}{\partial y_{3}}\left(x, \frac{b \theta}{2 \pi}+v(x)\right)\left(\frac{\partial v_{2}}{\partial \xi_{1}} \frac{\partial v_{3}}{\partial \xi_{2}}-\frac{\partial v_{3}}{\partial \xi_{1}} \frac{\partial v_{2}}{\partial \xi_{2}}\right)(x) d \mathcal{H}^{2}(x)
$$


We recall that $P_{n}=\left(\operatorname{cof} \nabla v_{n}\right) N \cdot s_{1}$. Since by Lemma 3.10 cof $\nabla v_{n} \in W^{1, t}\left(\Omega ; \mathbb{R}^{3 \times 3}\right)$ with $t=\frac{3 r}{6-r}$ by a trace theorem on $S_{t}$ we have $P_{n} \in W^{1-\frac{1}{t}, t}\left(S_{t} ; \mathbb{R}^{3 \times 3}\right)$, and by Sobolev embedding, $P_{n} \in L^{q}\left(S_{t} ; \mathbb{R}^{3 \times 3}\right)$ for any $q \leq \frac{9 r}{24-7 r}$. Moreover $\frac{\partial \varphi}{\partial y_{3}}\left(\cdot, \frac{b \theta}{2 \pi}+v_{n}(\cdot)\right)$ converges to $\frac{\partial \varphi}{\partial y_{3}}\left(\cdot, \frac{b \theta}{2 \pi}+v(\cdot)\right)$ in $W^{2-\frac{1}{r}, r}\left(S_{t} ; \mathbb{R}\right)$ and hence in $L^{s}\left(S_{t} ; \mathbb{R}\right)$ with $s=\frac{3 r}{4-2 r}$. Now the claim follows by Hölder inequality since $r>\frac{12}{7}>\frac{18}{11}$.

The treatment of the second and third lines in (4.14) is similar (see also Remark 4.4).

Step 2: Convergence of (4.8a). We have to prove that for all $\varphi \in \mathcal{C}_{c}^{\infty}\left(\Omega \times \mathbb{T}^{3}\right)$ it holds

$$
\mathcal{J}_{v_{n}}^{i, 1}(\varphi) \rightarrow \mathcal{J}_{v}^{i, 1}(\varphi)
$$

As for the previous step, we claim that

$$
\begin{aligned}
& \mathcal{J}_{v_{n}}^{i, 1}(\varphi)=\frac{|b|}{2 \pi}\left(\int_{0}^{2 \pi} \int_{S_{t}} \frac{\partial \varphi}{\partial x_{j}}\left(x, \frac{b \theta}{2 \pi}+v_{n}(x)\right)\left(A_{j 1}(x) A_{i 2}(x)-A_{j 2}(x) A_{i 1}(x)\right) d \mathcal{H}^{2}(x) d \theta\right. \\
& \left.+\int_{0}^{2 \pi} \int_{S_{t}} \frac{\partial \varphi}{\partial y_{j}}\left(x, \frac{b \theta}{2 \pi}+v_{n}(x)\right)\left(\frac{\partial\left(v_{n}\right)_{j}}{\partial \xi_{1}}(x) A_{i 2}(x)-\frac{\partial\left(v_{n}\right)_{j}}{\partial \xi_{2}}(x) A_{i 1}(x)\right) d \mathcal{H}^{2}(x) d \theta\right)
\end{aligned}
$$

converges to

$$
\begin{aligned}
& \frac{|b|}{(2 \pi)^{2}}\left(\int_{0}^{2 \pi} \int_{\Omega} \frac{\partial \varphi}{\partial x_{j}}\left(x, \frac{b \theta}{2 \pi}+v(x)\right)\left(A_{j 1}(x) A_{i 2}(x)-A_{j 2}(x) A_{i 1}(x)\right)|\nabla w(x)| d x d \theta\right. \\
& \left.+\int_{0}^{2 \pi} \int_{\Omega} \frac{\partial \varphi}{\partial y_{j}}\left(x, \frac{b \theta}{2 \pi}+v(x)\right)\left(\frac{\partial v_{j}}{\partial \xi_{1}}(x) A_{i 2}(x)-\frac{\partial v_{j}}{\partial \xi_{2}}(x) A_{i 1}(x)\right)|\nabla w(x)| d x d \theta\right) .
\end{aligned}
$$

Emulating the arguments of Step 1, the convergence of the first line is straightforward. To treat the second line, we observe that the quantity $\left(E_{n}\right)_{i j}:=\frac{\partial\left(v_{n}\right)_{j}}{\partial \xi_{2}}(x) A_{i 1}(x)-\frac{\partial\left(v_{n}\right)_{j}}{\partial \xi_{1}}(x) A_{i 2}(x)$ satisfies the relation

$$
\left(E_{n}\right)_{i j}=\left(e_{i} \times \nabla\left(v_{n}\right)_{j}\right) \cdot N=\left(\nabla\left(v_{n}\right)_{j} \times N\right) \cdot e_{i} .
$$

Indeed it suffices to recall that $e_{i}=A_{i k} s_{k}$, i.e., $e_{i}$ has coordinates $A_{i k}$ in the basis $\left\{s_{1}, s_{2}, s_{3}\right\}$, and to notice that $\left(E_{n}\right)_{i j}$ coincides with the third component of the vector $e_{i} \times \nabla\left(v_{n}\right)_{j}$, we recall that the third component of $\left\{s_{1}, s_{2}, s_{3}\right\}$ is parallel to $N$.

To prove the desired convergence it now suffices to argue as in Step 1. Moreover, (4.24) is seen to coincides with

$$
\begin{aligned}
& \mathcal{J}_{v}^{i, 1}(\varphi)=\frac{|b|}{2 \pi}\left(\int_{0}^{2 \pi} \int_{S_{t}} \frac{\partial \varphi}{\partial x_{j}}\left(x, \frac{b \theta}{2 \pi}+v(x)\right)\left(A_{j 1}(x) A_{i 2}(x)-A_{j 2}(x) A_{i 1}(x)\right) d \mathcal{H}^{2}(x) d \theta\right. \\
& \left.+\int_{0}^{2 \pi} \int_{S_{t}} \frac{\partial \varphi}{\partial y_{j}}\left(x, \frac{b \theta}{2 \pi}+v(x)\right)\left(\frac{\partial v_{j}}{\partial \xi_{1}}(x) A_{i 2}(x)-\frac{\partial v_{j}}{\partial \xi_{2}}(x) A_{i 1}(x)\right) d \mathcal{H}^{2}(x) d \theta\right)
\end{aligned}
$$

for any $t \in[0,2 \pi)$.

Step 3. Let us finally see that the value of $\mathcal{J}_{v}(\varphi)$ does depend only on the value of $\varphi$ in a neighborhood of $L \times \mathbb{T}^{3}$. This also follows from point (b) and the fact that, if $\tilde{\varphi}$ coincides with $\varphi$ in a neighborhood of $L \times \mathbb{T}^{3}$, then

$$
\mathcal{J}_{v}(\varphi-\tilde{\varphi})=\lim _{n \rightarrow+\infty} \mathcal{J}_{v_{n}}(\varphi-\tilde{\varphi})=0 .
$$


Remark 4.4. To see the convergence of the second line in (4.15) the argument is very similar to the one adopted for the first line, with the difference that this term have to be treated as for the last line in (4.24). Indeed the term

$$
\frac{\partial\left(v_{n}\right)_{2}}{\partial \xi_{2}} A_{j 1}-\frac{\partial\left(v_{n}\right)_{2}}{\partial \xi_{1}} A_{j 2}
$$

is recognized as the quantity $\left(\nabla\left(v_{n}\right)_{2} \times N\right) \cdot e_{j}$. Then it suffices to follow the argument in the proof of step 1 considering that $\nabla\left(v_{n}\right)_{2} \in W^{1, r}\left(\Omega ; \mathbb{R}^{3}\right)$ and $\nabla \varphi\left(\cdot, \frac{\theta b}{2 \pi}+v_{n}(\cdot)\right) \in W^{2, r}\left(\Omega ; \mathbb{R}^{3}\right)$ thanks to the regularity of $v_{n}$.

\section{The fundamental lemma}

In order to prove the following Lemma we need to introduce some additional notation. Let $L$ be a simple Lipschitz loop in $\Omega$ and introduce the distance function $d(x):=\operatorname{dist}(x, L)$ which is a Lipschitz function whose differential satisfies for a.e. $x \in \Omega,|\nabla d(x)|=1$. Given a function $v \in W^{2, r}\left(\Omega ; \mathbb{R}^{3}\right)$, we also introduce the current $\overline{\mathcal{L} \wedge b} \in \mathcal{D}_{2}\left(\Omega \times \mathbb{T}^{3}\right)$ defined as

$$
\overline{\mathcal{L} \wedge b}(\omega)=\frac{1}{2 \pi} \int_{0}^{2 \pi} \int_{S}\left\langle d \omega\left(x, \frac{b \theta}{2 \pi}+v(x)\right),\left(s_{1}, 0\right) \wedge\left(s_{2}, 0\right) \wedge \vec{b}\right\rangle d \mathcal{H}^{2}(x) d \theta
$$

for all $\omega \in \mathcal{D}^{2}\left(\Omega \times \mathbb{T}^{3}\right)$. In the formula above $S$ is an arbitrary Lipschitz surface enclosing $\mathcal{L}$, while, as usual, $\vec{b}=(0, b) \in \Lambda_{1} \mathbb{R}^{6}$.

Lemma 4.5. Let $L$ be a closed simple Lipschitz loop, and let $u=\left(u_{1}, u_{2}, u_{3}\right) \in S B V\left(\Omega ; \mathbb{R}^{3}\right) \cap$ $W^{1, p}\left(\Omega ; \mathbb{T}^{3}\right)$ be the harmonic map satisfying (3.12) with $b \in 2 \pi \mathbb{Z}^{3}$. Let $w \in W^{1, p}(\Omega ; \mathbb{T})$ be defined by $w \frac{b}{2 \pi}=u$. Let $v \in W^{2, r}\left(\Omega ; \mathbb{R}^{3}\right)$ and assume that $\nabla u \in L^{p}\left(\Omega ; \mathbb{R}^{3 \times 3}\right)$, with $\frac{6-2 r}{3 r}+\frac{1}{p} \leq 1$. If the following condition holds true:

$$
\lim _{\epsilon \rightarrow 0} \frac{1}{\epsilon} \int_{S_{t} \cap\{d \leq \epsilon\}}|\nabla v \times N| d x=0 \quad \text { for a.e. } t \in[0,2 \pi),
$$

where $N=\frac{\nabla w}{|\nabla w|}$ is the unit normal to $S_{t}$, then

$$
\mathcal{J}_{v}(\omega)=\overline{\mathcal{L} \wedge b}(\omega) .
$$

Proof. We shall prove that condition (4.28) has the property of nullifying some components of $\mathcal{J}_{v}(\omega)$. In particular, using the coordinates system introduced in (4.8), we will show that $\mathcal{J}_{v}^{0,1 i}(\varphi)=\mathcal{J}_{v}\left(\varphi d y_{1} \wedge d y_{i}\right)=0$, for all $\varphi \in \mathcal{C}_{c}^{\infty}\left(\Omega \times \mathbb{T}^{3}\right)$, for $i=2,3$. This will imply the thesis, thanks to the fact that the only nonzero components of $\mathcal{J}_{v}$ are those in (4.10) (compare with (4.9)). Let us recall that

$$
\begin{aligned}
& \mathcal{J}_{v}\left(\varphi d y_{1} \wedge d y_{i}\right)=\mathcal{J}_{v}^{0,1 i}(\varphi)= \\
& \frac{|b|}{2 \pi}\left((-1)^{i} \int_{0}^{2 \pi} \int_{S_{t}} \frac{\partial \varphi}{\partial y_{i^{c}}}\left(x, \frac{b \theta}{2 \pi}+v(x)\right)\left(\frac{\partial v_{2}}{\partial \xi_{1}} \frac{\partial v_{3}}{\partial \xi_{2}}-\frac{\partial v_{3}}{\partial \xi_{1}} \frac{\partial v_{2}}{\partial \xi_{2}}\right)(x) d \mathcal{H}^{2}(x) d \theta\right. \\
& \left.-\sum_{j=1}^{3} \int_{0}^{2 \pi} \int_{S_{t}} \frac{\partial \varphi}{\partial x_{j}}\left(x, \frac{b \theta}{2 \pi}+v(x)\right)\left(A_{j 1}(x) \frac{\partial v_{i}}{\partial \xi_{2}}(x)-A_{j 2}(x) \frac{\partial v_{i}}{\partial \xi_{1}}(x)\right) d \mathcal{H}^{2}(x) d \theta\right),
\end{aligned}
$$

for an arbitrary $t \in[0,2 \pi)$. Thanks to Lemma 4.3 (c) this expression does not depend on the value of $\varphi$ outside the neighborhood $\{d \leq \epsilon\} \times \mathbb{T}^{3}$ of $L \times \mathbb{T}^{3}$. Hence we consider a smooth 
cut-off function $\eta_{\epsilon}: \mathbb{R} \rightarrow[0, \infty)$ which is even and such that $\eta_{\epsilon}=1$ in $[0, \epsilon / 2]$, vanishes in $[\epsilon, \infty)$, and its derivatives satisfies $\left|\eta_{\epsilon}^{\prime}\right|<3 / \epsilon$. Then we set $\varphi(x, y)=\psi(x, y) \eta_{\epsilon}(d(x))$ with $\psi \in \mathcal{C}_{c}^{\infty}\left(\Omega \times \mathbb{T}^{3}\right)$ in the expression above and we estimate

$$
\begin{aligned}
& \left|\mathcal{J}_{v}^{0,1 j}(\varphi)\right| \leq \frac{|b|}{2 \pi} \int_{0}^{2 \pi} \int_{S_{t}} \eta_{\epsilon}(d(x))\left|\frac{\partial \psi}{\partial y_{i^{c}}}\left(x, \frac{b \theta}{2 \pi}+v(x)\right)\right|\left|\frac{\partial v_{2}}{\partial \xi_{1}} \frac{\partial v_{3}}{\partial \xi_{2}}-\frac{\partial v_{3}}{\partial \xi_{1}} \frac{\partial v_{2}}{\partial \xi_{2}}\right|(x) d \mathcal{H}^{2}(x) d \theta \\
& +\sum_{j=1}^{3} \frac{|b|}{2 \pi} \int_{0}^{2 \pi} \int_{S_{t}} \eta_{\epsilon}(d(x))\left|\frac{\partial \psi}{\partial x_{j}}\left(x, \frac{b \theta}{2 \pi}+v(x)\right)\right|\left|A_{j 1}(x) \frac{\partial v_{i}}{\partial \xi_{2}}(x)-A_{j 2}(x) \frac{\partial v_{i}}{\partial \xi_{1}}(x)\right| d \mathcal{H}^{2}(x) d \theta \\
& +\sum_{j=1}^{3} \frac{3|b|}{2 \pi \epsilon} \int_{0}^{2 \pi} \int_{S_{t} \cap\{d<\epsilon\}}\left|\psi\left(x, \frac{b \theta}{2 \pi}+v(x)\right)\right|\left|A_{j 1}(x) \frac{\partial v_{i}}{\partial \xi_{2}}(x)-A_{j 2}(x) \frac{\partial v_{i}}{\partial \xi_{1}}(x)\right| d \mathcal{H}^{2}(x) d \theta .
\end{aligned}
$$

The first term tends to 0 since $|J|:=\left|\frac{\partial v_{2}}{\partial \xi_{1}} \frac{\partial v_{3}}{\partial \xi_{2}}-\frac{\partial v_{3}}{\partial \xi_{1}} \frac{\partial v_{2}}{\partial \xi_{2}}\right|$ belongs to $L^{1}\left(S_{t}\right)$ while $\eta_{\epsilon} \downarrow 0$ $\mathcal{H}^{2}$-a.e. and $\nabla \psi$ is bounded. Indeed, $J$ is recognized as the determinant of the matrix

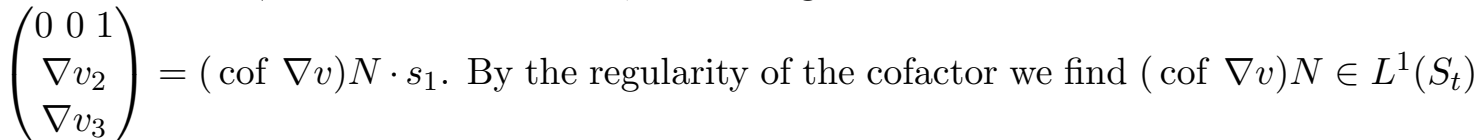
by standard theorem of traces for Sobolev spaces.

As for the other terms in (4.31), they also tend to zero, keeping into account condition (4.28). The product $A_{j 1}(x) \frac{\partial v_{i}}{\partial \xi_{2}}(x)-A_{j 2}(x) \frac{\partial v_{i}}{\partial \xi_{1}}(x)$ coincides with $\left(\nabla v_{i} \times N\right) \cdot e_{j}$, so the claim follows by the fact that the latter belongs to $L^{1}\left(S_{t}\right)$ and by (4.28).

In a similar way it is possible to prove that also the second line in $(4.8 \mathrm{a})$ vanishes. Hence we arrive at the following characterization of the current $\mathcal{J}_{v}$; namely, written in components, this reads

$$
\begin{aligned}
& \mathcal{J}_{v}\left(\varphi d x_{i} \wedge d y_{1}\right)=\frac{|b|}{2 \pi} \int_{0}^{2 \pi} \int_{S_{t}} \frac{\partial \varphi}{\partial x_{j}}\left(x, \frac{b \theta}{2 \pi}+v(x)\right)\left(A_{j 1}(x) A_{i 2}(x)-A_{j 2}(x) A_{i 1}(x)\right) d \mathcal{H}^{2}(x) d \theta, \\
& \mathcal{J}_{v}\left(\varphi d x_{i} \wedge d x_{j}\right)=\mathcal{J}_{v}\left(\varphi d y_{1} \wedge d y_{i}\right)=\mathcal{J}_{v}\left(\varphi d y_{2} \wedge d y_{3}\right)=0 .
\end{aligned}
$$

Now it suffices to recognize that this expression entails that $\mathcal{J}_{v}$ coincides with the expression in (4.27), by definition.

Looking back at (4.10) we find out that $\overline{\mathcal{L} \wedge b}$ is exactly $\mathcal{L} \wedge b$ when $v$ is of class $\mathcal{C}^{1}$. Arguing by approximation of $v$ by smooth maps $v_{n}$ we easily infer that the current (4.29) is well defined and its mass does not increase after approximation, since for every $n$ it coincides with $|\mathcal{L}||b|$, i.e.,

$$
M(\overline{\mathcal{L} \wedge b})=|\mathcal{L}||b| .
$$

Not to overburden notation we will still denote $\overline{\mathcal{L} \wedge b}$ by $\mathcal{L} \wedge b$ itself. We have obtained the following corollary:

Corollary 4.6. In the hypotheses of Lemma 4.5, it holds

$$
M\left(\partial \mathcal{G}_{u+v}\right)=M(\mathcal{L} \wedge b) .
$$

As a consequence $\mathcal{G}_{u+v}$ is an integral current in $\mathcal{D}_{3}\left(\Omega \times \mathbb{T}^{3}\right)$.

Remark 4.7. For a physical viewpoint condition (4.28) seems to be difficult to check. However, as we will see in the next section, this is strictly related to the summability of the cofactor of $\nabla u$. 


\subsection{Main result 1: characterization of the graph boundary for clusters with a single Burgers vector}

In this section, instead of a single line, we study dislocation clusters associated with a single Burgers vector $b \in 2 \pi \mathbb{Z}^{3}$. Let $L$ be a Lipschitz closed loop in $\Omega$. We know that the Minkowski content of $L$ tends to the 1-dimensional Hausdorff measure of $L$, that is

$$
\frac{|\{x \in \Omega: d(x, L)<r\}|}{\pi r^{2}} \rightarrow \mathcal{H}^{1}(L), \quad \text { as } r \rightarrow 0 .
$$

Let now $L$ be a countable union of closed Lipschitz curves $L_{i} \subset \Omega$ such that $\mathcal{H}^{1}(L)=$ $\sum_{i=1}^{\infty} \mathcal{H}^{1}\left(L_{i}\right)<+\infty$. For any $i \in \mathbb{N}$, by (4.33), there is a non-negative real number $r_{i}$ such that, if $r<r_{i}$, then it holds true

$$
\frac{\left|\left\{x \in \Omega: d\left(x, L_{i}\right)<r\right\}\right|}{\pi r^{2}}<2 \mathcal{H}^{1}\left(L_{i}\right) .
$$

Now, choose a sequence of non-negative real numbers $\delta_{i}, i \in \mathbb{N}$, as

$$
\delta_{i}:=\min \left\{2^{-1-i}, r_{i}\right\}<1,
$$

so that $\sum_{i=0}^{\infty} \delta_{i} \leq 1$. For $\epsilon>0$, let us define the open set $D_{\epsilon}$ as

$$
D_{\epsilon}:=\cup_{i \in \mathbb{N}} D_{\epsilon}^{i}, \quad D_{\epsilon}^{i}=\left\{x \in \Omega: d\left(x, L_{i}\right)<\epsilon \delta_{i}\right\} .
$$

By (4.34), one has

$$
\begin{aligned}
& \frac{\left|D_{\epsilon}^{i}\right|}{\pi \epsilon^{2} \delta_{i}^{2}}<2 \mathcal{H}^{1}\left(L_{i}\right) \quad \forall i \in \mathbb{N} \\
& \left|D^{\epsilon}\right| \leq \sum_{i=0}^{\infty}\left|D_{\epsilon}^{i}\right| \leq 2 \pi \epsilon^{2}\left(\sum_{i=0}^{\infty} \delta_{i}^{2} \mathcal{H}^{1}\left(L_{i}\right)\right) \leq 2 \pi \epsilon^{2} \mathcal{H}^{1}(L), \\
& \left|D^{\epsilon}\right| \rightarrow 0 \quad \text { as } \epsilon \rightarrow 0 .
\end{aligned}
$$

Let $\mathcal{L}$ be a closed integral current in $\mathcal{D}_{1}(\Omega)$. By Theorem 3.1 we infer that there is a sequence of indecomposable 1-currents $\mathcal{L}_{i}$ supported on Lipschitz loops $L_{i}$ such that $\mathcal{L}=\sum_{i=0}^{\infty} \mathcal{L}_{i}$. Moreover there exists an integral 2-current $\mathcal{S} \in \mathcal{D}_{2}(\Omega)$ with $\partial \mathcal{S}=\mathcal{L}$ that, again by decomposition Theorem, can be written as $\mathcal{S}=\sum_{i=0}^{\infty} \mathcal{S}_{i}$ with $\mathcal{S}_{i}$ undecomposable integral 2-currents with $\partial \mathcal{S}_{i}=\mathcal{L}_{i}$. We will now analyze the boundary of the graph of deformations of the form $\nabla u+\nabla v$ with $u$ being the solution to (3.12) with the cluster $\mathcal{L}$ in place of the simple loop $L$. It will be easy to see, in the case $v$ is of class $\mathcal{C}^{1}$, that the boundary $\partial \mathcal{G}_{u+v}$ will take the form

$$
\mathcal{J}_{v}(\omega)=\frac{1}{2 \pi} \int_{0}^{2 \pi} \int_{\mathcal{S}}\left\langle d \omega\left(x, \frac{b \theta}{2 \pi}+v(x)\right),\left(s_{1}, \frac{\partial v}{\partial s_{1}}(x)\right) \wedge\left(s_{2}, \frac{\partial v}{\partial s_{2}}(x)\right) \wedge \vec{b}\right\rangle d \mathcal{H}^{2}(x) d \theta,
$$

for all $\omega \in \mathcal{D}^{2}\left(\Omega \times \mathbb{T}^{3}\right)$ (see Lemma 4.8 below). Moreover we will see that the integral (4.38) does not depend on the specific current $\mathcal{S}$. Indeed, if $v$ is of class $\mathcal{C}^{1}$, we can apply Stokes Theorem as in (4.5) and (4.6).

Let us now characterize the boundary of $\mathcal{G}_{u+v}$ for general fields $v \in W^{2, r}\left(\Omega ; \mathbb{R}^{3}\right)$. 
Lemma 4.8. Let $\mathcal{L} \in \mathcal{D}_{1}(\Omega)$ be a closed integral 1 -current in $\Omega$, let $u \in S B V\left(\Omega ; \mathbb{R}^{3}\right) \cap$ $W^{1, p}\left(\Omega ; \mathbb{T}^{3}\right)$ be the harmonic map of Theorem 3.4, satisfying (3.12) with $b \in 2 \pi \mathbb{Z}^{3}$. Let $v \in W^{2, r}\left(\Omega ; \mathbb{R}^{3}\right)$ and assume

$$
r>\frac{12}{7}, \quad p<2, \quad \text { and } \quad \frac{6-2 r}{3 r}+\frac{1}{p} \leq 1 .
$$

Then the current given by the graph of $u+v$ with values in $\mathbb{T}^{3}$, namely $\mathcal{G}_{u+v} \in \mathcal{D}_{3}\left(\Omega \times \mathbb{T}^{3}\right)$, is an integer multiplicity current with finite mass and with boundary given by

$$
\partial \mathcal{G}_{u+v}(\omega)=\frac{1}{2 \pi} \int_{0}^{2 \pi} \int_{\mathcal{S}_{t}}\left\langle d \omega\left(x, \frac{b \theta}{2 \pi}+v(x)\right),\left(s_{1}, \frac{\partial v}{\partial s_{1}}(x)\right) \wedge\left(s_{2}, \frac{\partial v}{\partial s_{2}}(x)\right) \wedge \vec{b}\right\rangle d \mathcal{H}^{2}(x) d \theta
$$

for all $\omega \in \mathcal{D}^{2}\left(\Omega \times \mathbb{T}^{3}\right)$ and a.e. $t \in[0,2 \pi)$. Here $\mathcal{S}_{t} \in \mathcal{D}_{2}(\Omega)$ is an integral 2-current whose boundary is $\mathcal{L}$ and whose support is $S_{t}$, the level set of the map $w \in W^{1, p}(\Omega ; \mathbb{T})$, which, as in Lemma 4.3, is defined by $\frac{b}{2 \pi} w=u$.

Proof. The fact that the current $\mathcal{G}_{u+v}$ is rectifiable with integer multiplicity is an easy consequence of the fact that the graph

$$
G_{u+v}=\left\{(x, y) \in \Omega \times \mathbb{T}^{3}: y=u(x)+v(x)\right\}
$$

is a 3-rectifiable set (see Theorem 4 in [21, Section 3.1.5] and Proposition 1 in [21, Section 3.2.1]). Moreover it has finite mass since all the minors of $\nabla u+\nabla v$ are integrable, as a consequence of condition (4.39), taking into account that $\nabla u$ is of rank 1.

Let us prove that the boundary of $\mathcal{G}_{u+v}$ takes the form (4.40). Let $v_{n} \in \mathcal{C}^{\infty}\left(\bar{\Omega} ; \mathbb{R}^{3}\right)$ be a sequence such that $v_{n} \rightarrow v$ strongly in $W^{2, r}\left(\Omega ; \mathbb{R}^{3}\right)$. Let us write $\mathcal{L}$ as a countable sum of undecomposable components, $\mathcal{L}=\sum_{i} \mathcal{L}_{i}$, where $\mathcal{L}_{i}$ is a simple closed Lipschitz curve in $\Omega$ for all $i$. Accordingly let us decompose $\mathcal{S}_{t}$ in indecomposable components $\left(\mathcal{S}_{t}\right)_{i}$ in such a way that $\partial\left(\mathcal{S}_{t}\right)_{i}=\mathcal{L}_{i}$ (we recall that by Lemma 3.8 it holds $\partial \mathcal{S}_{t}=\mathcal{L}$ for a.e. $t \in[0,2 \pi$ ), and that moreover the multiplicity of $\mathcal{S}_{t}$ is 1 for a.e. $t \in[0,2 \pi)$ ). We will first show that for all $n>0$ the graph $\mathcal{G}_{u+v_{n}}$ has boundary given by

$$
\mathcal{J}_{v_{n}}(\omega):=\frac{1}{2 \pi} \int_{0}^{2 \pi} \int_{S_{t}}\left\langle d \omega\left(x, \frac{b \theta}{2 \pi}+v_{n}(x)\right),\left(s_{1}, \frac{\partial v_{n}}{\partial s_{1}}(x)\right) \wedge\left(s_{2}, \frac{\partial v_{n}}{\partial s_{2}}(x)\right) \wedge \vec{b}\right\rangle d \mathcal{H}^{2}(x) d \theta
$$

where $S_{t}=\{w=t \in[0,2 \pi)\}$ is an arbitrary level set of $w$. To see this we proceed as follows. Let $u_{i}$ be the function in $S B V\left(\Omega ; \mathbb{R}^{3}\right) \cap W^{1, \frac{3}{2}}\left(\Omega ; \mathbb{T}^{3}\right)$ given by (3.12) with $S_{t}$ replaced by $\left(S_{t}\right)_{i}$, whose boundary is $L_{i}$, so that $-\operatorname{Curl}\left(\nabla u_{i}\right)=b \otimes \mathcal{L}_{i}$. It is easy to see that $u^{m}:=\sum_{i=0}^{m} u_{i}$ converges to $u$ strongly in $W^{1, \frac{3}{2}}\left(\Omega ; \mathbb{T}^{3}\right)$, as $m \rightarrow \infty$; indeed we easily see that

$$
\nabla u^{m} \rightarrow \nabla u \quad \text { in } L^{\frac{3}{2}}\left(\Omega ; \mathbb{R}^{3 \times 3}\right),
$$

and thus, by Poincaré's inequality for torus-valued maps, $u^{m} \rightarrow u$ strongly in $L^{\frac{3}{2}}\left(\Omega ; \mathbb{T}^{3}\right)$ as $m \rightarrow \infty$.

Furthermore it is easy to see that $\mathcal{G}_{u^{m}+v_{n}}$ converges to $\mathcal{G}_{u+v_{n}}$ in the sense of currents as $m \rightarrow \infty$, thanks to the fact that $v_{n}$ is smooth, $\nabla u^{m}$ has rank 1 (i.e. that the cofactors 
and determinants are null), and the strong convergence of $u^{m}$. Defining

$$
\mathcal{J}_{v_{n}}^{m}(\omega):=\frac{1}{2 \pi} \int_{0}^{2 \pi} \int_{S_{t}^{m}}\left\langle d \omega\left(x, \frac{b \theta}{2 \pi}+v_{n}(x)\right),\left(s_{1}, \frac{\partial v_{n}}{\partial s_{1}}(x)\right) \wedge\left(s_{2}, \frac{\partial v_{n}}{\partial s_{2}}(x)\right) \wedge \vec{b}\right\rangle d \mathcal{H}^{2}(x) d \theta
$$

where $S_{t}^{m}=\left\{w^{m}=t\right\}\left(w^{m}\right.$ is defined via $\left.\frac{b}{2 \pi} w^{m}=u^{m}\right)$, we claim that $\mathcal{J}_{v_{n}}^{m}(\omega)$ converges to $\mathcal{J}_{v_{n}}(\omega)$ in (4.41) for all $\omega \in \mathcal{D}^{2}\left(\Omega \times \mathbb{T}^{3}\right)$ as $m \rightarrow+\infty$. Indeed, using the coarea formula as in (4.16) and (4.17) we see that

$$
\begin{aligned}
& \mathcal{J}_{v_{n}}^{m}(\omega)=\frac{1}{2 \pi} \int_{0}^{2 \pi} \mathcal{J}_{v_{n}}^{m}(\omega) d t= \\
& \frac{1}{(2 \pi)^{2}} \int_{0}^{2 \pi} \int_{\Omega}\left\langle d \omega\left(x, \frac{b \theta}{2 \pi}+v_{n}(x)\right),\left(s_{1}, \frac{\partial v_{n}}{\partial s_{1}}(x)\right) \wedge\left(s_{2}, \frac{\partial v_{n}}{\partial s_{2}}(x)\right) \wedge \vec{b}\right\rangle\left|\nabla w^{m}(x)\right| d x d \theta .
\end{aligned}
$$

Thanks to convergence (4.42), the smoothness of $v_{n}$ and (4.39), this converges as $m \rightarrow \infty$ to

$$
\begin{aligned}
& \frac{1}{(2 \pi)^{2}} \int_{0}^{2 \pi} \int_{\Omega}\left\langle d \omega\left(x, \frac{b \theta}{2 \pi}+v_{n}(x)\right),\left(s_{1}, \frac{\partial v_{n}}{\partial s_{1}}(x)\right) \wedge\left(s_{2}, \frac{\partial v_{n}}{\partial s_{2}}(x)\right) \wedge \vec{b}\right\rangle|\nabla w(x)| d x d \theta= \\
& \frac{1}{2 \pi} \int_{0}^{2 \pi} \mathcal{J}_{v_{n}}(\omega) d t=\mathcal{J}_{v_{n}}(\omega),
\end{aligned}
$$

where in the first equality we have used again the coarea formula and in the last equality the fact that the integral in (4.41) does not depend on $t \in[0,2 \pi)$, thanks to the regularity of $v_{n}$ (we can apply Stokes Theorem together with Lemma 3.8). Now we want to pass to the limit $\mathcal{J}_{v_{n}}(\omega)$ as $n$ tends to $\infty$. Using again the coarea formula, as in the last expression we have

$$
\mathcal{J}_{v_{n}}(\omega)=\frac{1}{(2 \pi)^{2}} \int_{0}^{2 \pi} \int_{\Omega}\left\langle d \omega\left(x, \frac{b \theta}{2 \pi}+v_{n}(x)\right),\left(s_{1}, \frac{\partial v_{n}}{\partial s_{1}}(x)\right) \wedge\left(s_{2}, \frac{\partial v_{n}}{\partial s_{2}}(x)\right) \wedge \vec{b}|\nabla w|\right\rangle d x d \theta .
$$

Thanks to the condition on the coefficients (4.39), this tends to

$$
\frac{1}{(2 \pi)^{2}} \int_{0}^{2 \pi} \int_{\Omega}\left\langle d \omega\left(x, \frac{b \theta}{2 \pi}+v(x)\right),\left(s_{1}, \frac{\partial v}{\partial s_{1}}(x)\right) \wedge\left(s_{2}, \frac{\partial v}{\partial s_{2}}(x)\right) \wedge \vec{b}|\nabla w|\right\rangle d x d \theta,
$$

which again by coarea formula equals

$$
\begin{aligned}
& \mathcal{J}_{v}(\omega):= \\
& \frac{1}{(2 \pi)^{2}} \int_{0}^{2 \pi} \int_{0}^{2 \pi} \int_{S_{t}}\left\langle d \omega\left(x, \frac{b \theta}{2 \pi}+v(x)\right),\left(s_{1}, \frac{\partial v}{\partial s_{1}}(x)\right) \wedge\left(s_{2}, \frac{\partial v}{\partial s_{2}}(x)\right) \wedge \vec{b}\right\rangle d \mathcal{H}^{2}(x) d t d \theta,
\end{aligned}
$$

where $S_{t}:=\{w=t\}, t \in[0,2 \pi)$.

Let us now show that the quantity

$$
J_{t}:=\int_{0}^{2 \pi} \int_{S_{t}}\left\langle d \omega\left(x, \frac{b \theta}{2 \pi}+v(x)\right),\left(s_{1}, \frac{\partial v}{\partial s_{1}}(x)\right) \wedge\left(s_{2}, \frac{\partial v}{\partial s_{2}}(x)\right) \wedge \vec{b}\right\rangle d \mathcal{H}^{2}(x) d \theta
$$

does not depend on $t \in[0,2 \pi)$. This will demonstrate that

$$
\mathcal{J}_{v}(\omega)=\frac{1}{2 \pi} \int_{0}^{2 \pi} \int_{S_{t}}\left\langle d \omega\left(x, \frac{b \theta}{2 \pi}+v(x)\right),\left(s_{1}, \frac{\partial v}{\partial s_{1}}(x)\right) \wedge\left(s_{2}, \frac{\partial v}{\partial s_{2}}(x)\right) \wedge \vec{b}\right\rangle d \mathcal{H}^{2}(x) d \theta
$$


for a.e. $t \in[0,2 \pi)$.

For a.e. $\bar{t} \in[0,2 \pi)$, by Lemma (3.8) the surface $S_{\bar{t}}$ has boundary $L$, and by decomposition writes as $S_{\bar{t}}=\sum_{i}\left(S_{\bar{t}}\right)_{i}$ with $\partial\left(S_{\bar{t}}\right)_{i}=L_{i}$. Setting

$$
J_{\bar{t}}^{i}:=\int_{0}^{2 \pi} \int_{\left(S_{\bar{t}}\right)_{i}}\left\langle d \omega\left(x, \frac{b \theta}{2 \pi}+v(x)\right),\left(s_{1}, \frac{\partial v}{\partial s_{1}}(x)\right) \wedge\left(s_{2}, \frac{\partial v}{\partial s_{2}}(x)\right) \wedge \vec{b}\right\rangle d \mathcal{H}^{2}(x) d \theta,
$$

it follows that $J_{\bar{t}}=\sum_{i} J_{\bar{t}}^{i}$, and $J_{\bar{t}}^{i}$ is recognized as $\mathcal{J}_{v}(\omega)^{i}$, the boundary of $\mathcal{G}_{u_{i}+v}$, which by Lemma 4.3 does not depend on the specific surface $\left(S_{\bar{t}}\right)_{i}$. In particular we infer $J_{\bar{t}}^{i}=$ $\mathcal{J}_{v}(\omega)^{i}=J_{t}^{i}$, so that it follows $J_{\bar{t}}=\sum_{i} J_{\bar{t}}^{i}=\sum_{i} J_{t}^{i}=J_{t}$. The thesis follows.

We will now refine Lemma 4.5.

Lemma 4.9. Let $\mathcal{L} \in \mathcal{D}_{1}(\Omega)$ be a closed integral current in $\Omega$, let $u=\left(u_{1}, u_{2}, u_{3}\right) \in$ $S B V\left(\Omega ; \mathbb{R}^{3}\right) \cap W^{1, p}\left(\Omega ; \mathbb{T}^{3}\right)$ be the harmonic map satisfying (3.12) with $b \in \mathbb{Z}^{3}$, and let $w \in W^{1, p}(\Omega ; \mathbb{T})$ be defined by $\frac{b}{2 \pi} w=u$. Let $v \in W^{2, r}\left(\Omega ; \mathbb{R}^{3}\right)$ and assume that (4.39) is satisfied. Let $\mathcal{L}$ decompose as $\mathcal{L}=\sum_{i=0}^{\infty} \mathcal{L}_{i}$, with $\mathcal{L}_{i}$ simple Lipschitz closed curves in $\Omega$, let $\left\{\delta_{i}\right\}_{i \in \mathbb{N}}$ the sequence of non-negative numbers in (4.35), and define $D_{\epsilon}$ by (4.36). Assume that

$$
\lim _{\epsilon \rightarrow 0} \frac{1}{\epsilon} \int_{S_{t} \cap D_{\epsilon}}|\nabla v \times N| d \mathcal{H}^{2}(x)=0
$$

for a.e. $t \in[0,2 \pi)$, where $N=\frac{\nabla w}{|\nabla w|}$ is the unit normal to the level set $S_{t}=\{w=t\}$. Then

$$
\mathcal{J}_{v}(\omega)=\mathcal{L} \wedge b(\omega)=\sum_{i} \mathcal{L}_{i} \wedge b(\omega)
$$

for all $\omega \in \mathcal{D}^{2}\left(\Omega \times \mathbb{T}^{3}\right)$. As a consequence $\mathcal{G}_{u+v}$ is an integral current in $\mathcal{D}_{3}\left(\Omega \times \mathbb{T}^{3}\right)$ whose boundary is $\mathcal{L} \wedge$ b.

Proof. From Lemma 4.8 we infer that, if (4.48) holds true, then $\mathcal{G}_{u+v}$ is an integral current whose boundary is $\partial \mathcal{G}_{u+v}=\mathcal{L} \wedge b$. Then we have only to prove that (4.47) implies (4.48). We will achieve this by means of Lemma 4.5. The current $\mathcal{S}_{t}$ can be decomposed as follows:

$$
\mathcal{S}_{t}=\cup_{i=0}^{\infty}\left(\mathcal{S}_{t}\right)_{i} \quad \text { with } \partial\left(\mathcal{S}_{t}\right)_{i}=\mathcal{L}_{i},
$$

and, thanks to $(3.17),\left(\mathcal{S}_{t}\right)_{i}$ are essentially disjoint surfaces for a.e. $t \in[0,2 \pi)$. Hence we deduce that

$$
\mathcal{J}_{v}(\omega)=\sum_{i=0}^{\infty} \mathcal{J}_{v}^{i}(\omega)
$$

with

$$
\mathcal{J}_{v}^{i}(\omega)=\frac{1}{2 \pi} \int_{0}^{2 \pi} \int_{\left(\mathcal{S}_{t}\right)_{i}}\left\langle d \omega\left(x, \frac{b \theta}{2 \pi}+v(x)\right),\left(s_{1}, \frac{\partial v}{\partial s_{1}}(x)\right) \wedge\left(s_{2}, \frac{\partial v}{\partial s_{2}}(x)\right) \wedge \vec{b}\right\rangle d \mathcal{H}^{2}(x) d \theta,
$$

for all $\omega \in \mathcal{D}^{2}\left(\Omega \times \mathbb{T}^{3}\right)$. We will prove that

$$
\mathcal{J}_{v}^{i}=\mathcal{L}_{i} \wedge b \quad \forall i \in \mathbb{N},
$$


which will achieve the thesis. To see (4.51) we estimate, for fixed $i \in \mathbb{N}$,

$$
\int_{\left(\mathcal{S}_{t}\right)_{i} \cap D_{\epsilon}^{i}}|\nabla v \times N| d \mathcal{H}^{2}(x) \leq \int_{\mathcal{S}_{t} \cap D_{\epsilon}}|\nabla v \times N| d \mathcal{H}^{2}(x)
$$

and thus, by (4.47),

$$
\frac{1}{\epsilon} \int_{\left(\mathcal{S}_{t}\right)_{i} \cap D_{\epsilon}^{i}}|\nabla v \times N| d \mathcal{H}^{2}(x) \rightarrow 0 \quad \text { as } \epsilon \rightarrow 0 .
$$

This allows us to employ the same argument of Lemma 4.5 which provides (4.51), and the thesis follows.

Remark 4.10. We emphasize the intrinsic difficulty to check, from a practical viewpoint, the assumption (4.47). The surface $\mathcal{S}_{t}$ a priori has not a direct physical interpretation. However, as we will see, condition (4.47) is readily guaranteed as soon as the cofactor of the deformation $F$ is at least square integrable. This will be clear in the following theorem.

We will now prove the following crucial result:

Theorem 4.11 (Main result). Let $\mathcal{L} \in \mathcal{D}_{1}(\Omega)$ be a closed integral current, let $u=$ $\left(u_{1}, u_{2}, u_{3}\right) \in S B V\left(\Omega ; \mathbb{R}^{3}\right) \cap W^{1, p}\left(\Omega ; \mathbb{T}^{3}\right)$ be the harmonic map satisfying (3.12) with $b \in 2 \pi \mathbb{Z}^{3}$. Let $v \in W^{2, r}\left(\Omega ; \mathbb{R}^{3}\right)$ and suppose $\nabla u \in L^{p}\left(\Omega ; \mathbb{R}^{3 \times 3}\right)$ with

$$
\frac{6-2 r}{3 r}+\frac{1}{p} \leq 1
$$

Finally, let $F=\nabla u+\nabla v$ and assume that cof $F \in L^{2}\left(\Omega ; \mathbb{R}^{3 \times 3}\right)$. Then the graph $\mathcal{G}_{u+v}$ is an integral current in $\mathcal{D}_{3}\left(\Omega \times \mathbb{T}^{3}\right)$ whose boundary is given by

$$
\begin{aligned}
& \partial \mathcal{G}_{u+v}(\omega)=\mathcal{L} \wedge b(\omega)= \\
& \frac{1}{2 \pi} \int_{0}^{2 \pi} \int_{S_{t}}\left\langle d \omega\left(x, \frac{b \theta}{2 \pi}+v(x)\right),\left(s_{1}, 0\right) \wedge\left(s_{2}, 0\right) \wedge \vec{b}\right\rangle d \mathcal{H}^{2}(x) d \theta
\end{aligned}
$$

for all $\omega \in \mathcal{D}^{2}\left(\Omega \times \mathbb{T}^{3}\right)$ and a.e. $t \in[0,2 \pi)$.

Proof. In order to prove Theorem 4.11 we will show that the property cof $F \in L^{2}\left(\Omega ; \mathbb{R}^{3 \times 3}\right)$ implies that condition (4.47) holds true, and hence the thesis will follow by virtue of Lemma 4.9. Let us define the sequence $\left\{\delta_{i}\right\}_{i \in \mathbb{N}}$ and the set $D_{\epsilon}$ as in (4.35) and (4.36); we write for any $j=1,2,3$,

$$
\begin{aligned}
& \frac{1}{2 \pi \epsilon} \int_{0}^{2 \pi} \int_{\mathcal{S}_{t} \cap D_{\epsilon}}\left|\nabla v_{j} \times N\right| d \mathcal{H}^{2}(x) d t=\frac{1}{2 \pi \epsilon} \int_{0}^{2 \pi} \int_{\mathcal{S}_{t} \cap D_{\epsilon}}\left|\nabla v_{j} \times \frac{\nabla w}{|\nabla w|}\right| d \mathcal{H}^{2}(x) d t= \\
& \frac{1}{2 \pi \epsilon} \int_{D_{\epsilon}}\left|\nabla v_{j} \times \nabla w\right| d x \leq \frac{1}{2 \pi \epsilon} \int_{D_{\epsilon}}|\operatorname{cof} F| d x,
\end{aligned}
$$

where in the second equality we employed the coarea formula, while in the last one we used that $\left|\nabla v_{j} \times \nabla w\right| \leq|\operatorname{cof} F|$ for any $j=1,2,3$. Now, by Schwarz inequality,

$$
\frac{1}{2 \pi \epsilon} \int_{D_{\epsilon}}|\operatorname{cof} F| d x \leq \frac{1}{2 \pi \epsilon}\left|D_{\epsilon}\right|^{1 / 2}\left(\int_{D_{\epsilon}}|\operatorname{cof} F|^{2} d x\right)^{1 / 2} \leq C\left(\int_{D_{\epsilon}}|\operatorname{cof} F|^{2} d x\right)^{1 / 2},
$$


with $C:=\frac{\sqrt{2 \pi \mathcal{H}^{1}(L)}}{2 \pi}$ and where we have used (4.37). Now, since cof $F \in L^{2}\left(\Omega ; \mathbb{R}^{3 \times 3}\right)$ and $\left|D_{\epsilon}\right| \rightarrow 0$ as $\epsilon \rightarrow 0$, we conclude that the quantity in the right-hand side of (4.56), and hence also $\frac{1}{2 \pi \epsilon} \int_{0}^{2 \pi} \int_{\mathcal{S}_{t} \cap D_{\epsilon}}|\nabla v \times N| d \mathcal{H}^{2}(x) d t$, by (4.55), vanishes as $\epsilon \rightarrow 0$. As a consequence we infer that for a.e. $t \in[0,2 \pi)$

$$
\lim _{\epsilon \rightarrow 0} \frac{1}{\epsilon} \int_{\mathcal{S}_{t} \cap D_{\epsilon}}|\nabla v \times N| d \mathcal{H}^{2}(x)=0
$$

and the thesis follows.

Let us go back to (4.1) and (4.8). For regular field $v$, we have seen that

$$
\mathcal{C}_{u+v}\left(\varphi d y_{1} \wedge d y_{i}\right)=\frac{|b|}{2 \pi} \int_{0}^{2 \pi} \int_{L} \varphi\left(x, \frac{b \theta}{2 \pi}+v(x)\right) \frac{\partial v_{i}}{\partial \tau}(x) d \mathcal{H}^{2}(x) d \theta, \quad i \in\{2,3\}
$$

for any $\varphi \in \mathcal{C}^{\infty}\left(\Omega \times \mathbb{R}^{3}\right)$ which is $2 \pi$-periodic in the second variable. In particular we get the following Corollary:

Corollary 4.12. Assume the hypotheses of Lemma 4.9 and suppose $v$ is of class $\mathcal{C}^{1}\left(\bar{\Omega} ; \mathbb{R}^{3}\right)$. Then it holds

$$
\frac{\partial v}{\partial \tau} \times b=0 \quad \text { on } L
$$

In particular, this happens under the hypotheses of Theorem 4.11 and $v \in \mathcal{C}^{1}\left(\bar{\Omega} ; \mathbb{R}^{3}\right)$.

\subsection{Main result 2: characterization of the graph boundary for clusters with general Burgers vectors}

In this section we study generalizations of some results in the previous Section to deformations whose curl is a dislocation measure with associated Burgers vectors spanning whole $2 \pi \mathbb{Z}^{3}$. Determining the current associated to the graph of such deformations is a hard task that we currently consider as far from reach. However it is possible to prove that this graph is an integral current in some specific cases.

Let $\mathcal{L}_{1}, \mathcal{L}_{2}, \mathcal{L}_{3} \in \mathcal{D}_{1}(\Omega)$ three closed integral currents and let $\mathcal{L}$ be the regular dislocation current whose components are $\mathcal{L}_{i}, i=1,2,3$, according to (3.20). This is equivalent to say that $\mathcal{L}_{i}$ is the dislocation cluster associated to the Burgers vector $2 \pi e_{i}, i=1,2,3$. In particular we set

$$
\Lambda_{\mathcal{L}}:=\sum_{i=1}^{3} \Lambda_{i}=2 \pi \sum_{i=1}^{3} \mathcal{L}_{i} \otimes e_{i},
$$

and assume that $u_{i}: \Omega \rightarrow \mathbb{T}^{3}$ is the displacement field generating $\Lambda_{i}$, i.e. $u_{i}$ is the map given by (3.12) with $b=2 \pi e_{i}$ and $L$ replaced by $\mathcal{L}_{i}$, so that

$$
-\operatorname{Curl} \nabla u_{i}=\Lambda_{i}^{T} \text {. }
$$

Let us denote by $u=u_{1}+u_{2}+u_{3}$. We introduce the following currents $\mathcal{J}_{v+u}^{i} \in \mathcal{D}_{2}\left(\Omega \times \mathbb{T}^{3}\right)$, $i=1,2,3$,

$$
\mathcal{J}_{v+u}^{i}(\omega):=\int_{0}^{2 \pi} \int_{S_{t}^{i}}\left\langle d \omega\left(x, e_{i} \theta+\hat{u}_{i}(x)\right),\left(s_{1}, \frac{\partial \hat{u}_{i}}{\partial s_{1}}(x)\right) \wedge\left(s_{2}, \frac{\partial \hat{u}_{i}}{\partial s_{2}}(x)\right) \wedge \vec{e}_{i}\right\rangle d \mathcal{H}^{2}(x) d \theta
$$


for any $\omega \in \mathcal{D}^{2}\left(\Omega \times \mathbb{T}^{3}\right)$, where $S_{t}^{i}=\left\{w_{i}=t\right\}$ is any level set of the function $w_{i}$ defined via $e_{i} w_{i}=u_{i}(i=1,2,3)$, and where we have set

$$
\hat{u}_{i}:=u+v-u_{i}, \quad i=\{1,2,3\} .
$$

In (4.61) we have denoted $\overrightarrow{e_{i}}=\left(0, e_{i}\right) \in \Lambda_{1} \mathbb{R}^{6}$ as usual.

Theorem 4.13. Let $\mathcal{L}_{i} \in \mathcal{D}_{1}(\Omega), i=1,2,3$, be closed integral currents whose supports $L_{i}$ are disjoint. In particular, let us assume that $d\left(L_{i}, L_{j}\right)>0$ for $i \neq j$; for $i=1,2,3$ let $u_{i} \in W^{1, p}\left(\Omega ; \mathbb{T}^{3}\right)$ be the harmonic map given by (3.12) with $L=L_{i}$ and $b=2 \pi e_{i}$, and let $v \in W^{2, r}\left(\Omega ; \mathbb{R}^{3}\right)$. Suppose that

$$
r>\frac{12}{7}, \quad p \in\left(\frac{3}{2}, 2\right) \quad \text { and } \quad \frac{6-2 r}{3 r}+\frac{1}{p} \leq 1 .
$$

Then $\mathcal{G}_{u+v}$ is an integer multiplicity current whose boundary satisfies

$$
\partial \mathcal{G}_{u+v}(\omega)=\sum_{i=1}^{3} \mathcal{J}_{v+u}^{i}(\omega),
$$

for any $\omega \in \mathcal{D}^{2}\left(\Omega \times \mathbb{T}^{3}\right)$.

Proof. This is a straightforward consequence of Lemma 4.8. In fact, let $U_{i} \supset L_{i}, i=1,2,3$, be three disjoint open sets, neighborhoods of the supports $L_{i}$ (which exist thanks to the hypothesis $d\left(L_{i}, L_{j}\right)>0$ for $\left.i \neq j\right)$. Hence the graph of $u+v$ is the sum of the following restrictions

$$
\mathcal{G}_{u+v}=\mathcal{G}_{u+v}\left\llcorner U_{1}+\mathcal{G}_{u+v}\left\llcorner U_{2}+\mathcal{G}_{u+v}\left\llcorner U_{3}+\mathcal{G}_{u+v}\left\llcorner U^{c},\right.\right.\right.\right.
$$

with $U^{c}=\Omega \backslash\left(\cup_{i=1}^{3} U_{i}\right)$. Let us focus on $U_{1}$ (similarly we will argue for $U_{2}$ and $U_{3}$ ); we know that $u_{2}$ and $u_{3}$ are smooth in $\bar{U}_{1}$, so that $\hat{u}_{1}=u_{2}+u_{3}+v \in W^{2, r}\left(U_{1} ; \mathbb{R}^{3}\right)$. In particular this function can be extended to a (non-relabeled) function $\hat{u}_{1} \in W^{2, r}\left(\Omega ; \mathbb{R}^{3}\right)$ and Lemma 4.8 applies, implying that

$$
\partial \mathcal{G}_{u+v}\left\llcorner U_{1}(\omega)=\int_{0}^{2 \pi} \int_{S_{t}^{1} \cap U_{1}}\left\langle d \omega\left(x, \frac{b \theta}{2 \pi}+\hat{u}_{1}(x)\right),\left(s_{1}, \frac{\partial \hat{u}_{1}}{\partial s_{1}}(x)\right) \wedge\left(s_{2}, \frac{\partial \hat{u}_{1}}{\partial s_{2}}(x)\right) \wedge \vec{e}_{1}\right\rangle d \mathcal{H}^{2}(x) d \theta,\right.
$$

for any $\omega \in \mathcal{D}^{2}\left(\Omega \times \mathbb{T}^{3}\right)$. Since the value of this expression depends only on the value of $\omega$ in $U_{1}$ the thesis easily follows, by (4.65).

Theorem 4.14. Under the hypotheses of Theorem 4.13, let us assume in addition that the tensor field $F:=\sum_{i=1}^{3} \nabla u_{i}+\nabla v \in W^{1, p}\left(\Omega ; \mathbb{R}^{3 \times 3}\right)$ satisfies the property that cof $F \in$ $L^{2}\left(\Omega ; \mathbb{R}^{3 \times 3}\right)$. Then the graph $\mathcal{G}_{u+v}$ is an integral current in $\mathcal{D}_{3}\left(\Omega \times \mathbb{T}^{3}\right)$ whose boundary is

$$
\begin{aligned}
& \partial \mathcal{G}_{u+v}(\omega)=2 \pi \sum_{i=1}^{3} \mathcal{L}_{i} \wedge e_{i}(\omega)= \\
& \sum_{i=1}^{3} \int_{0}^{2 \pi} \int_{S_{t}^{i}}\left\langle d \omega\left(x, e_{i} \theta+\hat{u}_{i}(x)\right),\left(s_{1}, 0\right) \wedge\left(s_{2}, 0\right) \wedge \vec{e}_{i}\right\rangle d \mathcal{H}^{2}(x) d \theta,
\end{aligned}
$$

for all $\omega \in \mathcal{D}^{3}\left(\Omega \times \mathbb{T}^{3}\right)$.

Proof. This is an easy consequence of Theorem 4.11; indeed, arguing as in Theorem 4.13, we focus on the open set $U_{1}$ and establish that the boundary of $\mathcal{G}_{u+v}$ restricted to $U_{1}$ is exactly $2 \pi \mathcal{L}_{1} \wedge e_{1}$. The same holds true on the other sets $U_{2}$ and $U_{3}$. Notice that it is here crucial to assume that $d\left(L_{i}, L_{j}\right)>0$ for $i \neq j$. 


\section{Minimization problems}

\subsection{The minimization setting}

The mechanical setting is the following: we assume $\Omega$ to be a bounded and simply connected open set in $\mathbb{R}^{3}$ which represents a single crystal. Let $\hat{\Omega}$ be another bounded open set such that $\Omega \subset \subset \hat{\Omega}$. We fix a dislocation density on $\partial \Omega$ by prescribing a kinematical boundary condition accordingly in terms of the deformation tensor $\hat{F}$ in $\hat{\Omega}$. No other traction boundary condition is assumed. We then consider dislocation microstructures as resulting from a variational problem with these boundary conditions in a unloaded regime. Let us emphasize that the external field $\hat{F}$ is not necessarily in equilibrium. Indeed this boundary condition is equivalent to a Dirichlet boundary condition, since we can always write the external field $\hat{F}$ as the gradient of a suitable torus-valued map $\tilde{u}$, and then fixing $\hat{F}$ corresponds to fix $\tilde{u}$, as done in [34]. It was shown in [31, Section 5.4] that (essentially due to the solenoidal property of the dislocation density) the solution is not the trivial one (i.e., the absence of dislocations in $\Omega$ ).

Boundary conditions. We will prescribe Dirichlet boundary conditions for the deformation field $F$ and for the dislocation cluster as follows. We fix a tensor field $\hat{F} \in L^{p}\left(\hat{\Omega} ; \mathbb{R}^{3 \times 3}\right)$ and a regular dislocation current $\hat{\alpha}$ having support in $\hat{\Omega}$ and such that

$$
-\operatorname{Curl} \hat{F}=\Lambda_{\hat{\alpha}}^{T},
$$

where $\Lambda_{\hat{\alpha}}$ is the dislocation density measure introduced in Definition 3.9. Then we say that a field $F \in L^{p}\left(\hat{\Omega} ; \mathbb{R}^{3 \times 3}\right)$ is admissible for the boundary condition if

(i) $F=\hat{F} \quad$ on $\hat{\Omega} \backslash \Omega$,

(ii) there is a dislocation current $\alpha$ such that $\alpha=\hat{\alpha}$ on $\hat{\Omega} \backslash \Omega$ and $-\operatorname{Curl} F=\Lambda_{\alpha}^{T}$.

Energy. We consider an energy functional which depends on the tensor field $F$ and on its derivatives as follows. The stored-elastic energy is represented by a functional $W_{\mathrm{e}}(M(F))$, with $M(F)$ the vector of minors of $F$ as introduced in (3.2). The total elastic energy is given by the integral over $\Omega$ of $W_{\mathrm{e}}(M(F))$, namely

$$
\mathcal{W}_{\mathrm{e}}(M(F))=\int_{\Omega} W_{\mathrm{e}}(M(F)) d x
$$

We assume that

(A1) $\mathcal{W}_{\mathrm{e}}$ is lower semi-continuous with respect to the convergence of $F$, cof $F$, and $\operatorname{det} F$ in $L^{p}\left(\Omega ; \mathbb{R}^{3 \times 3}\right), L^{q}\left(\Omega ; \mathbb{R}^{3 \times 3}\right)$, and $L^{s}(\Omega)$, respectively.

We will also assume the following growth condition on $W_{\mathrm{e}}$ : there are constants $C, c>0$, and $\delta \geq 0$ such that

(A2) $W_{\mathrm{e}}(M(F)) \geq C\left(|F|^{p}+|\operatorname{cof} F|^{q}+\delta|\operatorname{det} F|^{s}\right)-c$,

for some coefficient $p, q, s>1$ to be specified later. Notice that in order to guarantee (A1) we can assume $\mathcal{W}_{e}$ polyconvex, i.e. $W_{\mathrm{e}}$ is a convex function of $M(F)$ (see, e.g., [7,12]).

The total energy of the system also contains higher order terms, depending on the derivatives of $F$. The defect part of the energy encodes the quantity of dislocation singularities. This is an energetic term denoted by $\mathcal{W}_{\text {dislo }}$ which depends on the dislocation density of $F$, i.e. on $\operatorname{Curl} F$. We will make the following assumption on $\mathcal{W}_{\text {dislo }}$ 
(A3) $\mathcal{W}_{\text {dislo }}$ is l.s.c. with respect to the weak star convergence of measures,

and the following growth condition

$$
\mathcal{W}_{\text {dislo }}\left(\Lambda_{\mathcal{L}}\right) \geq C\left|\Lambda_{\mathcal{L}}\right|(\Omega)-c
$$

for some positive constants $C$ and $c$. We will also assume that the total energy depends on $\operatorname{Div} F$ via the following high order term $\mathcal{W}_{\mathrm{d}}$ which satisfies $\mathcal{W}_{\mathrm{d}}(\operatorname{Div} F)=$ $\int_{\Omega} W_{\mathrm{d}}(\operatorname{Div} F) d x$, and

(A5) $\mathcal{W}_{\mathrm{d}}$ is l.s.c. with respect to the weak convergence of $L^{r}\left(\Omega ; \mathbb{R}^{3}\right)$,

(A6) $\quad W_{\mathrm{d}}(\operatorname{Div} F) \geq C|\operatorname{Div} F|^{r}-c$,

for some positive constants $C$ and $c$. The total energy of a deformation field $F$ reads

$$
\mathcal{W}(F, \nabla F)=\mathcal{W}_{\mathrm{e}}(M(F))+\mathcal{W}_{\text {dislo }}(\operatorname{Curl} F)+\mathcal{W}_{\mathrm{d}}(\operatorname{Div} F)
$$

and satisfies the coercivity condition

$$
\mathcal{W}(F, \nabla F) \geq C\left(\|F\|_{L^{p}}^{p}+\|\operatorname{cof} F\|_{L^{q}}^{q}+\delta\|\operatorname{det} F\|_{L^{s}}^{s}+\|\operatorname{Div} F\|_{L^{r}}^{r}\right)+c\left|\Lambda_{\mathcal{L}}\right|(\Omega)-\gamma,
$$

for suitable positive constant $C, c, \gamma$, and $\delta \geq 0$, depending on the material properties.

In the case that the dislocation cluster is associated with a single Burgers vector, by Lemma 3.11 it follows that even if $\delta=0$, the energy satisfies the following coercivity condition with respect to the determinant of $F$,

$$
\mathcal{W}(F, \nabla F) \geq \tilde{C}\|\operatorname{det} F\|_{L^{t}}^{t}-\tilde{\gamma},
$$

with $\frac{1}{t}:=\frac{6-2 r}{3 r}+\frac{1}{p}<1$, for some positive constants $\tilde{C}$ and $\tilde{\gamma}$. By formula

$$
I \operatorname{det} F=F^{T} \operatorname{cof} F \text {, }
$$

it can be seen that the same coercivity (5.5) holds true also in the case $\frac{6-2 r}{3 r}+\frac{1}{p}=1$, by assuming $\frac{1}{t}:=\frac{1}{p}+\frac{1}{q}<1$.

Remark 5.1. The presence of the energetic term $\mathcal{W}_{\text {dislo }}$ together with the boundary condition $\hat{\alpha}$ for the dislocation prevents the following minimum problem to have trivial solutions where the dislocation cluster of the minimizer is null. Indeed in the definition of admissibility it is required that any competitor for the minimum problem has a suitable dislocation cluster where Curl $F$ concentrates. What might happen is that such dislocation cluster moves, along a minimizing sequence, up to the boundary of the crystal, hence provoking that the minimizer has a cluster concentrated on $\partial \Omega$. However, according to the geometry of the crystal, this solution often is excluded because energetically inconvenient. In [32, Section 5.4] we show that in the most relevant cases the boundary condition forces the dislocation cluster of the minimizer to remain inside the crystal. 


\subsection{Problem 1: dislocation clusters with linearly dependent Burgers vectors}

In this section we study a minimization problem among a class of deformations around dislocation clusters whose Burgers vectors are all multiple of a fixed Burgers vector $b \in$ $2 \pi \mathbb{Z}^{3}$. In this case, thanks to the results obtained in Section 4.3 it is seen that, under suitable conditions on the coefficients related to the growth of the energy (5.4), such deformations can all be seen as gradients of suitable maps with values in the torus $\mathbb{T}^{3}$ and whose graph is an integral current in the space $\Omega \times \mathbb{T}^{3}$. As a consequence we can rely to Theorem 3.3 in order to get compactness of minimizing sequences in this class of deformations, and hence to show the existence of minimizers.

Introduce the class of admissible deformation fields as follows:

$$
\mathcal{F}_{b}:=\left\{F \in L^{p}\left(\hat{\Omega} ; \mathbb{R}^{3 \times 3}\right) \text { satisfying conditions (1), (2), (3) below }\right\} .
$$

(1) $\operatorname{cof} F \in L^{q}\left(\hat{\Omega} ; \mathbb{R}^{3 \times 3}\right)$, det $F \in L^{s}(\hat{\Omega})$, Div $F \in L^{r}\left(\hat{\Omega} ; \mathbb{R}^{3}\right)$;

(2) - Curl $F=b \otimes \mathcal{L}$ for some integral closed and compactly supported current $\mathcal{L} \in$ $\mathcal{D}_{1}(\hat{\Omega})$

(3) $F=\hat{F}$ on $\hat{\Omega} \backslash \Omega$,

with $\hat{F}$ standing for the deformation boundary condition as introduced in (5.1), for any prescribed dislocation $\hat{\alpha}$ within $\hat{\Omega}$. First, let us observe that $F$ can be always written as

$$
F=\nabla u+\nabla v
$$

for some functions $u \in W^{1, p}\left(\hat{\Omega} ; \mathbb{T}^{3}\right)$ and $v \in W^{2, r}\left(\hat{\Omega} ; \mathbb{R}^{3}\right)$. This can be easily seen by Helmholtz decomposition, as shown in [33] and discussed in (3.25). We insist on this point since it is crucial for the proof of our existence results. As described in (3.22) and (3.24), we first decompose $F=\nabla w+F^{0}$ with $\Delta w=\operatorname{Div} F \in L^{r}\left(\Omega ; \mathbb{R}^{3}\right)$ and $\partial_{N} w=0$ on $\partial \hat{\Omega}$, and then $F^{0}=\nabla u^{0}+\nabla u$ with $u$ be the harmonic function given by (3.12), and $u_{0}$ being a harmonic function in $\hat{\Omega}$ with boundary conditions $\partial_{N} u_{0}=F \cdot N-\partial_{N} u=g-\partial_{N} u$. Since by assumption $g \in \mathcal{C}^{1, \alpha}$ and since the dislocation current $\mathcal{L}$ is compactly supported inside $\hat{\Omega}$, it turns out that $\partial_{N} u$ is of class $\mathcal{C}^{\infty}\left(\partial \hat{\Omega} ; \mathbb{R}^{3}\right)$, so that $g-\partial_{N} u$ is of class $\mathcal{C}^{1, \alpha}$ and by problem (3.10) it follows that $u_{0} \in \mathcal{C}^{2, \alpha}\left(\overline{\hat{\Omega}} ; \mathbb{R}^{3}\right)$. In particular setting $v:=w+u_{0}$ entails $v \in W^{2, r}\left(\hat{\Omega} ; \mathbb{R}^{3}\right)$, with boundary condition

$$
\partial_{N} v=g-\partial_{N} u
$$

Now, Theorem 4.11 ensures that if $F \in \mathcal{F}_{b}$ has coefficients satisfying

$$
\begin{aligned}
& r>\frac{12}{7}, \quad p<2, \\
& \frac{6-2 r}{3 r}+\frac{1}{p} \leq 1, \\
& s>1, \\
& q \geq 2,
\end{aligned}
$$


then $F$ is such that the graph of $u+v$, seen as a current $\mathcal{G}_{u+v} \in \mathcal{D}_{3}\left(\hat{\Omega} \times \mathbb{T}^{3}\right)$, is integral. Moreover its boundary takes the form

$$
\begin{aligned}
& \partial \mathcal{G}_{u+v}(\omega)=\mathcal{L} \wedge b(\omega)= \\
& \frac{1}{2 \pi} \int_{0}^{2 \pi} \int_{S}\left\langle d \omega\left(x, \frac{b \theta}{2 \pi}+v(x)\right),\left(s_{1}, 0\right) \wedge\left(s_{2}, 0\right) \wedge \vec{b}\right\rangle d \mathcal{H}^{2}(x) d \theta,
\end{aligned}
$$

for all $\omega \in \mathcal{D}^{2}\left(\hat{\Omega} \times \mathbb{T}^{3}\right)$, where $S$ is the support of the integral 2-current $\mathcal{S}$ with $\partial \mathcal{S}=\mathcal{L}$, which is a level set of $\tilde{u}$ (defined by $\frac{b}{2 \pi} \tilde{u}:=u$ ), and $\left\{s_{1}, s_{2}\right\}$ is an orthonormal basis for its tangent plane.

The minimum problem we are interested in is then the following: let $\hat{F} \in \mathcal{F}_{b}$ be fixed, we want to find a solution to

$$
\min _{F \in \mathcal{F}_{b}} \mathcal{W}(F, \nabla F)
$$

where $\mathcal{W}$ takes the form (5.3). We also make the following technical assumption:

$$
\text { either } \delta>0 \text { in (A2) or } \min \left\{\frac{6-2 r}{3 r}+\frac{1}{p}, \frac{1}{p}+\frac{1}{q}\right\}<1 \text {. }
$$

We can state our main result:

Theorem 5.2. Fix as dislocation boundary condition a regular dislocation current $\hat{\alpha}$ having support in $\hat{\Omega}$. Let $\hat{F} \in \mathcal{F}_{b}$ be fixed according to (5.1), and assume that the coefficients appearing in conditions (1) and (2) satisfy (5.9), (5.10), (5.11), and (5.12). Assume $\mathcal{W}$ is a functional on $\mathcal{F}_{b}$ as in (5.3) and satisfying conditions (A1)-(A6) and (5.15). Then there exists a minimizer $F \in \mathcal{F}_{b}$ of the problem (5.14).

The proof of Theorem 5.2 is standard and very similar to the one of Theorem 4.1 (see Theorems 6.6. and 6.8. in [33]). For this reason we here sketch it without details.

Proof. Let $F_{n}$ be a minimizing sequence in $\mathcal{F}_{b}$ for the problem (5.14). By (5.4) we infer the existence of $F \in L^{p}\left(\hat{\Omega} ; \mathbb{R}^{3 \times 3}\right), A \in L^{q}\left(\hat{\Omega} ; \mathbb{R}^{3 \times 3}\right), G \in L^{r}\left(\hat{\Omega} ; \mathbb{R}^{3}\right)$, and $\Lambda_{\mathcal{L}} \in \mathcal{M}_{b}\left(\hat{\Omega} ; \mathbb{R}^{3 \times 3}\right)$ such that

$$
\begin{aligned}
& F_{n} \rightarrow F \text { weakly in } L^{p}\left(\hat{\Omega} ; \mathbb{R}^{3 \times 3}\right), \\
& \text { cof } F_{n} \rightarrow A \text { weakly in } L^{q}\left(\hat{\Omega} ; \mathbb{R}^{3 \times 3}\right), \\
& \text { Div } F_{n} \rightarrow G \text { weakly in } L^{r}\left(\hat{\Omega} ; \mathbb{R}^{3}\right), \\
& \Lambda_{\mathcal{L}_{n}} \rightarrow \Lambda_{\mathcal{L}} \text { weak* in } \mathcal{M}_{b}\left(\hat{\Omega} ; \mathbb{R}^{3 \times 3}\right) .
\end{aligned}
$$

Moreover we find $D \in L^{m}(\hat{\Omega})$ such that

$$
\operatorname{det} F_{n} \rightarrow D \text { weakly in } L^{m}(\hat{\Omega}),
$$

with $m=s>1$ if $\delta>0$, and $m=t>1$ if $\delta=0$ (by condition (5.5), that in turn is ensured by (5.15)). Our aim is now to show that $A=\operatorname{cof} F, D=\operatorname{det} F, G=\operatorname{Div} F$, and - Curl $F=\Lambda_{\mathcal{L}}^{T}$ with $\Lambda_{\mathcal{L}}$ being a dislocation density of the form $\mathcal{L} \otimes b$ for some integral 1-current $\mathcal{L}$. The Dirichlet boundary conditions are easily seen to be satisfied. In order to see that $\Lambda_{\mathcal{L}}^{T}=b \otimes \mathcal{L}$ we follows the argument in [33, Theorem 6.6. and 6.8.] which relies on 
the application of compactness theorem for integral currents. The conditions $G=\operatorname{Div} F$, and - Curl $F=\Lambda_{\mathcal{L}}$ follow easily from the theory of distributions.

In order to prove that $A=\operatorname{cof} F, D=\operatorname{det} F$ we first show (once again as in [33, Theorem 6.6. and 6.8.]) that $F_{n}=\nabla v_{n}+\nabla u_{n}$ and $F=\nabla v+\nabla u$ with $v_{n} \in W^{2, r}\left(\hat{\Omega} ; \mathbb{R}^{3}\right)$ converging to $v \in W^{2, r}\left(\hat{\Omega} ; \mathbb{R}^{3}\right)$ weakly in this space and $u_{n} \in W^{1, p}\left(\hat{\Omega} ; \mathbb{T}^{3}\right)$ converging weakly to $u \in W^{1, p}\left(\hat{\Omega} ; \mathbb{T}^{3}\right)$. Then the conclusion will follow by applying Theorem 3.3, which can be used thanks to Theorem 4.11 that indeed provides a uniform bound for the boundaries $\partial \mathcal{G}_{v_{n}+u_{n}}$.

\subsection{Problem 2: dislocation clusters with general Burgers vectors}

In this Section we address a more general existence result valid for a larger class of deformations, which may show a general regular dislocation density, i.e. with general and possibly linearly independent Burgers vectors. To obtain compactness of minimizing sequence, we rely on the closedness of admissible deformations. To this aim, we need an additional hypothesis on the admissibility, which turns out to be non-necessary in case of linearly dependent Burgers vectors, where Theorem 4.11 provides such closedness, whereas in the case of general clusters, a characterization of admissible deformation fields in term of graphs is yet beyond reach.

The class of admissible deformation fields is the following:

$$
\mathcal{F}:=\left\{F \in L^{p}\left(\hat{\Omega} ; \mathbb{R}^{3 \times 3}\right) \text { satisfying conditions (1b), (2b), (3b), (4b) below }\right\} .
$$

(1b) $\operatorname{cof} F \in L^{q}\left(\hat{\Omega} ; \mathbb{R}^{3 \times 3}\right)$, det $F \in L^{s}(\hat{\Omega})$, Div $F \in L^{r}\left(\hat{\Omega} ; \mathbb{R}^{3}\right)$;

(2b) - Curl $F=\Lambda_{\mathcal{L}}^{T}$ for some regular dislocation current compactly supported in $\hat{\Omega}$. In particular, by decomposition, this can be written

$$
-\operatorname{Curl} F=\Lambda_{\mathcal{L}_{1}}^{T}+\Lambda_{\mathcal{L}_{2}}^{T}+\Lambda_{\mathcal{L}_{3}}^{T}, \quad \Lambda_{\mathcal{L}_{i}}=2 \pi \mathcal{L}_{i} \otimes e_{i}, i=1,2,3
$$

(3b) $F=\hat{F}$ on $\hat{\Omega} \backslash \Omega$, with $\hat{F}$ standing for the deformation boundary condition as introduced in (5.1), for any prescribed dislocation $\hat{\alpha}$ within $\hat{\Omega}$.

(4b) $F=\nabla u+\nabla v$ has the property that $\mathcal{G}_{u+v}$ is an integral current in $\mathcal{D}_{3}\left(\hat{\Omega} ; \mathbb{T}^{3}\right)$ whose boundary satisfies

$$
\begin{aligned}
\partial \mathcal{G}_{u+v}(\omega) & =2 \pi \sum_{i=1}^{3} \mathcal{L}_{i} \wedge e_{i}(\omega) \\
& =\sum_{i=1}^{3} \int_{0}^{2 \pi} \int_{S_{t}^{i}}\left\langle d \omega\left(x, e_{i} \theta+\hat{u}_{i}(x)\right),\left(s_{1}, 0\right) \wedge\left(s_{2}, 0\right) \wedge \overrightarrow{e_{i}}\right\rangle d \mathcal{H}^{2}(x) d \theta
\end{aligned}
$$

for all $\omega \in \mathcal{D}^{2}\left(\Omega \times \mathbb{T}^{3}\right)$.

Let us recall that $\hat{u}_{i}$ has been defined in (4.62) and $S_{t}^{i}=\left\{w_{i}=t\right\}$ with $e_{i} w_{i}=u_{i}$.

The main result regarding existence of minimizers for (5.14) with general Burgers vectors is the following: 
Theorem 5.3. Fix as dislocation boundary condition a regular dislocation current $\hat{\alpha}$ having support in $\hat{\Omega}$. Let $\hat{F} \in \mathcal{F}_{b}$ be fixed according to (5.1), and assume that the coefficients appearing in conditions (1) and (2) satisfy (5.9), (5.11), and $q>1$. Assume $\mathcal{W}$ is a functional on $\mathcal{F}$ as in (5.3) and satisfying conditions (A1)-(A6) with either $\delta>0$ or $\frac{1}{p}+\frac{1}{q}<1$. Then there exists a minimizer $F \in \mathcal{F}$ of the minimum problem

$$
\min _{F \in \mathcal{F}} \mathcal{W}(F, \nabla F)
$$

We drop the proof since it is a straightforward adaptation of the one of Theorem 5.2. As for the case $\delta=0$, it suffices to observe that the condition $\frac{1}{p}+\frac{1}{q}=: \frac{1}{s}<1$ provides an uniform bound of $\operatorname{det} F$ in $L^{s}(\Omega)$ via the identity $I \operatorname{det} F=F^{T}$ cof $F$ and by Hölder inequality.

Remark 5.4. Let us emphasize that once we assume (4b) we no longer need the assumption on the coefficient $q \geq 2$. Indeed in the case of the previous section, where the Burgers vectors are linearly dependent, this condition implies directly expression (5.13) of $\partial \mathcal{G}_{u+v}$ thanks to Theorem 4.11. In the general case we do not know if this expression takes place in general, so we have to make the assumption (4b). At the same time we know that (4b) is satisfied in some specific cases when $q \geq 2$ and the dislocations clusters show particular geometries, as Theorem 4.14 demonstrates.

Remark 5.5 (Admissible geometries). Condition (4b) and hence Theorem 5.3 holds as soon as the dislocation set consists of clusters (in its most general sense of closed integral currents) lying on glide planes each of which possesses a single Burgers vector. These structure are known as prismatic loops [23]. The admissible geometries are such that the glide planes must be disjoint if they are associated to non-parallel Burgers vectors. Note that there are at most three independent Burgers vectors and hence three sets of disjoint planes. Obviously the case of screw or edge dislocations, which are the only straight parallel dislocations [38], do comply with this condition.

\subsection{A problem involving $F$ and $F^{-1}$ and modeling discussion}

In the pioneer paper [26] as based on [28], the meaning of $F$ is that of the elastic part of the deformation tensor. In contrast, according to Acharya [1,2,4], and specifically discussed in [3], the physical meaning of $F$ should be that of the inverse deformation tensor, i.e., $F_{i j}(t)=\frac{\partial X_{i}}{\partial x_{j}}(t)$ with $x$ the position vector in the current configuration $\Omega$ at time $t$, whereas $X$ stands for the position of the material point in some "reference" configuration (this standpoint was already considered by the authors in [32]). Indeed, the dislocation density $\Lambda$ has a meaning only in $\Omega$, being equal to (the transpose) of - Curl $F$ (in [3], the meaning of $F$ is that of the inverse of the elastic part of the deformation tensor). It is the purpose of this section to show that our approach fits the two interpretations. In particular we propose a variant of the aforementioned minimization problems where the energy involves the variable

$$
G:=F^{-1} .
$$

The general problem of elasticity with dislocations can be formulated, according to [3, Eq. (4)] and in the absence of body forces, as follows: find $F$ and $G$ such that

$$
\Lambda=(-\operatorname{Curl} F)^{T}, \quad \text { Div } \mathbb{P}=0(+ \text { b.c. }), \quad \mathbb{P}=\hat{\mathbb{P}}(G) .
$$


Well posedness for this system is to the knowledge of the authors yet an open problem, in its full generality. With our approach, we are able to address existence in the case of a model of hyper-elasticity, i.e., with $\mathbb{P}=\partial_{G} \tilde{W}_{\mathrm{e}}(G)$. Specifically, we consider a general stored-elastic energy density (strain energy per unit volume of the current configuration) of the type

$$
\bar{W}_{\mathrm{e}}(F, G)=W_{\mathrm{e}}(F)+\tilde{W}_{\mathrm{e}}(G),
$$

together with higher-order terms involving $D F$ in the form of its curl, $\Lambda=-(\operatorname{Curl} F)^{T}$, and its divergence, Div $F$. Thus, we discuss existence results for a variational problem where the total energy takes the form

$$
\overline{\mathcal{W}}(F, D F, G):=\overline{\mathcal{W}}_{\mathrm{e}}(F, G)+\mathcal{W}_{\text {dislo }}(\operatorname{Curl} F)+\mathcal{W}_{\mathrm{d}}(\operatorname{Div} F),
$$

with $\overline{\mathcal{W}}_{\mathrm{e}}(F, G):=\int_{\Omega} \bar{W}_{\mathrm{e}}(F, G) d x$. To achieve this aim, we first observe that $G$ can be expressed as combinations of entries of the vector $M(F)$, namely

$$
G=\frac{(\operatorname{cof} F)^{T}}{\operatorname{det} F} .
$$

Moreover the energy depends on the dislocation density $\Lambda=-(\operatorname{Curl} F)^{T}$. Therefore, after considering a minimizing sequence $\left(F_{k}, G_{k}\right)$, we realize that we need a suitable control of the variable $F_{k}$ in order to guarantee that at the limit we have

$$
\text { if } \quad F_{k} \rightarrow F, \quad \text { then } \quad \Lambda_{k} \rightarrow \Lambda=-(\operatorname{Curl} F)^{T} .
$$

At the same time we need a sufficiently good control of $(\operatorname{cof} F)^{T}$ and $\operatorname{det} F$ in order to guarantee that

$$
\text { if } G_{k} \rightarrow G, \quad \text { then } \quad G=F^{-1}=\frac{(\operatorname{cof} F)^{T}}{\operatorname{det} F} .
$$

Hence, the only assumption we require is coercivity in the following sense: there exist $p_{1}, p_{2}, p_{3}, p_{4}, p_{5}>1$ chosen appropriately (see Subsection 5.2), with in addition $p_{4}>p_{2}^{\prime}=$ $\frac{p_{2}}{p_{2}-1}$ such that

$$
\overline{\mathcal{W}}(F, D F, G) \geq C\left(\|F\|_{p_{1}}^{p_{1}}+\|\operatorname{cof} F\|_{p_{2}}^{p_{2}}+\delta\|\operatorname{det} F\|_{p_{3}}^{p_{3}}+\|\operatorname{det} G\|_{p_{4}}^{p_{4}}+\|\operatorname{Div} F\|_{p_{5}}^{p_{5}}\right)-c,
$$

with $\delta \geq 0$, and $C, c>0$. Moreover we assume $\overline{\mathcal{W}}_{\mathrm{e}}(F, G)$ as $L^{1}$-lower semicontinuous (in particular the energy density depending on $M(F)$, that is $W_{\mathrm{e}}(F)$, might still be assumed polyconvex, but need not). Note that the energy density depending on $G$ can even be nonconvex and non-polyconvex provided it results that $\overline{\mathcal{W}}_{\mathrm{e}}(F, G)$ is $L^{1}$-lower semicontinuous. Further, to avoid matter interpenetration, it is assumed that $\bar{W}(F, G) \rightarrow+\infty$ as $\operatorname{det} G \rightarrow$ 0 (for, it suffices that $W_{\mathrm{e}}(F) \rightarrow+\infty$ as $\operatorname{det} F \rightarrow+\infty$ ).

The energy term $W_{\mathrm{e}}(F)$ should be understood as specific to our variational model of nonlinear bodies with dislocations, whereas in the absence of the dislocations the energy reduces to a term depending on the sole deformation gradient $G$.

Whereas the issue (5.24) is proved as in the previous variational problems, the main issue to work with is to prove (5.25). We can prove that

$$
G_{k}=\left(F_{k}\right)^{-1}=\frac{\left(\operatorname{cof} F_{k}\right)^{T}}{\operatorname{det} F_{k}} \rightarrow G=\frac{(\operatorname{cof} F)^{T}}{\operatorname{det} F}
$$


weakly in $L^{1}$ as $k \rightarrow \infty$. To this aim it is required a strong convergence of either $\left(\operatorname{cof} F_{k}\right)^{T}$ or $\operatorname{det} F_{k}$. The remaining part of this section is dedicated to prove that, under suitable choice of the coefficients $p_{1}, p_{2}, p_{3}, p_{4}, p_{5}>1$ and in the case of a dislocation cluster depending on one fixed Burgers vector, condition (5.27) holds true with

$$
\text { cof } F_{k} \rightarrow \text { cof } F \text { strongly in } L^{q}\left(\Omega ; \mathbb{R}^{3 \times 3}\right) \text { for all } q<p_{2} .
$$

As we have seen the deformation tensor is decomposed as the sum of two gradients, namely

$$
F=\nabla u+\nabla v
$$

where $u \in W^{1, p_{1}}\left(\Omega ; \mathbb{T}^{3}\right)$ and $v \in W^{2, p_{5}}\left(\Omega ; \mathbb{R}^{3}\right)$, satisfy

$$
\left\{\begin{array}{ll}
\operatorname{Div} \nabla u & =0 \\
-\operatorname{Curl} \nabla u & =b \otimes \mathcal{L}
\end{array}, \quad\left\{\begin{array}{ll}
\Delta v & =\operatorname{Div} F \\
-\operatorname{Curl} \nabla v & =0
\end{array},\right.\right.
$$

together with suitable boundary conditions. We have seen that $u$ satisfies $\|\nabla u\|_{L^{3 / 2}(\Omega)} \leq$ $c|\mu|(\Omega)$. Moreover, it was proved in [25] and [39] that ${ }^{2} \nabla u \in W^{s, p}\left(\Omega ; \mathbb{R}^{3 \times 3}\right)$ with $\frac{1}{p}=\frac{2+s}{3}$, $1<p<3 / 2,0<s<1$ and

$$
\|\nabla u\|_{W^{s, p}(\Omega)} \leq c|\mu|(\Omega) .
$$

Thus by compact embedding theorems [16], any bounded sequence $(\nabla u)_{k}$ in $W^{s, p}\left(\Omega ; \mathbb{R}^{3 \times 3}\right)$ converges up to a subsequence strongly in $L^{1}\left(\Omega ; \mathbb{R}^{3 \times 3}\right)$.

Lemma 5.6. In the case of one Burgers vector, assume the growth condition (5.26), and assume cof $F_{k} \rightarrow$ cof $F$ weakly in $L^{p_{2}}\left(\Omega ; \mathbb{R}^{3 \times 3}\right)$. Then cof $F_{k} \rightarrow$ cof $F$ strongly in $L^{q}\left(\Omega ; \mathbb{R}^{3 \times 3}\right)$ for every $q<p_{2}$. As a consequence, $(5.27)$ holds if $p_{4}>p_{2}^{\prime}=\frac{p_{2}}{p_{2}-1}$.

Proof. Thanks to the control of Div $F$ we know $\nabla v_{k} \in W^{1, p_{5}}\left(\Omega ; \mathbb{R}^{3}\right)$. By Sobolev embed$\operatorname{ding} \nabla v_{k} \in L^{3 p_{5} /\left(3-p_{5}\right)}\left(\Omega ; \mathbb{R}^{3 \times 3}\right)$, so that by Hölder inequality it is easy to see that (recall notation $(2.4))$

$$
\nabla v_{k} \otimes \nabla v_{k} \in W^{1,3 p_{5} /\left(6-p_{5}\right)}\left(\Omega ; \mathbb{R}^{3 \times 3}\right) .
$$

By compact embedding, one has

$$
\nabla v_{k} \otimes \nabla v_{k} \rightarrow \nabla v \otimes \nabla v
$$

strongly in $L^{l}\left(\Omega ; \mathbb{R}^{3 \times 3}\right)$ for $l<3 p_{5} /\left(6-2 p_{5}\right)$.

Moreover $\nabla u_{k} \rightarrow \nabla u$ strongly in $L^{m}\left(\Omega ; \mathbb{R}^{3 \times 3}\right)$ for all $m<3 / 2$, by virtue of (5.30). From $\nabla v_{k} \rightarrow \nabla v$ strongly in $L^{s}\left(\Omega ; \mathbb{R}^{3 \times 3}\right)$ for all $s<3 p_{5} /\left(3-p_{5}\right)$ Hölder's inequality yields

$$
\nabla v_{k} \otimes \nabla u_{k} \rightarrow \nabla v \otimes \nabla u
$$

strongly in $L^{t}\left(\Omega ; \mathbb{R}^{3 \times 3}\right)$ with $t$ such that $\frac{1}{t}>\frac{1}{s}+\frac{2}{3}$.

The proof proceeds by recalling the identity cof $F=\frac{1}{2} F \otimes F$ and by virtue of (5.29), with

$$
\operatorname{cof} F_{k} \rightarrow \operatorname{cof} F
$$

strongly in $L^{q}(\Omega)$ with $q:=\min \{l, t\}$. The assumption of a single Burgers vector is here crucial, since in the above product we have no occurrence of $\nabla u_{k} \otimes \nabla u_{k}$ for which strong convergence would not hold.

The thesis follows since, by the energy control of cof $F$ in $L^{p_{2}}\left(\Omega ; \mathbb{R}^{3 \times 3}\right)$ we have strong convergence in $L^{q}\left(\Omega ; \mathbb{R}^{3 \times 3}\right)$ for all $q<p_{2}$.

\footnotetext{
${ }^{2}$ The proof was established with the domain being the whole space $\Omega=\mathbb{R}^{3}$, but can be extended for bounded simply connected domains with smooth boundary as well, using the techniques of [10].
} 
Due to the presence of both the issues (5.24) and (5.25), at the present stage it seems a difficult task to deal with a variational problem involving only the variables Curl $F$ and $G$. One possibility is to consider an energy depending on $M(G)$ and Curl $F$, and using the expression

$$
F^{T}=\frac{\operatorname{cof} G}{\operatorname{det} G},
$$

in order to show that Curl $F_{k} \rightarrow \operatorname{Curl} F$. However, if the bulk energy depends on cof $G$ and $\operatorname{det} G$ it is a difficult task to show that

$$
\operatorname{cof} G_{k} \rightarrow \operatorname{cof} G, \quad \operatorname{det} G_{k} \rightarrow \operatorname{det} G .
$$

Indeed, these convergences, as for the corresponding convergences of cof $F$ and $\operatorname{det} F$, require a suitable regularity on the graph of the displacement $\tilde{w}$, whenever we can write $G=\nabla \tilde{w}$. This requires a suitable theory on the graphs of such functions, which is only provided in the present paper for the inverse matrix $F=G^{-1}$, exploiting the condition on Curl $F$ being a suitable integral current as related to the density of dislocations. The problem of the analysis of the graphs related to $G$ will be the topic of future investigations.

\section{Acknowledgements}

The authors are grateful and thank the anonymous referees for their careful reading of the manuscript and for their suggestions which allowed to strongly improve the paper. The authors also thank Jean Van Schaftingen for a key discussion on div-curl systems. This work has been supported by the iFCT project MATH2DISLOC.

\section{References}

[1] A. Acharya. A model of crystal plasticity based on the theory of continuously distributed dislocations. Journal of the Mechanics and Physics of Solids, 49(4):761 $784,2001$.

[2] A. Acharya. Constitutive analysis of finite deformation field dislocation mechanics. Journal of the Mechanics and Physics of Solids, 52(2):301 - 316, 2004.

[3] A. Acharya. A counterpoint to cermelli and gurtin's criteria for choosing the 'correct' geometric dislocation tensor in finite plasticity. In B. Daya Reddy, editor, IUTAM Symposium on Theoretical, Computational and Modelling Aspects of Inelastic Media, pages 99-105, Dordrecht, 2008. Springer Netherlands.

[4] A. Acharya. Microcanonical entropy and mesoscale dislocation mechanics and plasticity. Journal of Elasticity, 104(1):23-44, Aug 2011.

[5] F. Ahmad. Invariants of a Cartesian tensor of rank 3. Arch. Mech., 63(4):383-392, 2011.

[6] S. Amstutz and N. Van Goethem. The incompatibility operator: from Riemann's intrinsic view of geometry to a new model of elasto-plasticity. CIM Series in Mathematical Sci., J. F. Rodrigues and M. Hintermller eds. 
[7] J. M. Ball. Convexity conditions and existence theorems in nonlinear elasticity. Arch. Ration. Mech. Anal., 63:337-403, 1977.

[8] A. Bertram. Finite gradient elasticity and plasticity: a constitutive mechanical framework. Contin. Mech. Thermodyn., 27(6):1039-1058, 2015.

[9] J. Bourgain and A. Brezis. New estimates for the Laplacian, the divcurl, and related Hodge systems. Comptes Rendus Matematique, 338(I):539543, 2004.

[10] H. Brezis and J. Van Schaftingen. Boundary estimates for elliptic systems with $L^{1}$ data. Calc. Var. Partial Differ. Equ., 30(3):369-388, 2007.

[11] P. G. Ciarlet. An introduction to differential geometry with applications to elasticity. J. Elasticity, 78-79(1-3):3-201, 2005.

[12] B. Dacorogna. Direct methods in the calculus of variations. Applied Mathematical Sciences. Springer, 2008.

[13] G. Dal Maso and R. Toader. A model for the quasi-static growth of brittle fractures: Existence and approximation results. Arch. Ration. Mech. Anal., 162(2):101-135, 2002 .

[14] T. De Pauw and R. Hardt. Rectifiable and flat G chains in metric spaces. Amer. J. Math., 134(1):1-69, 2012.

[15] T. De Pauw and R. Hardt. Some basic theorems on flat G chains. J. Math. Anal. Appl., 418(2):1047-1061, 2014.

[16] E. Di Nezza, G. Palatucci, and E. Valdinoci. Hitchhiker's guide to the fractional Sobolev spaces. Bull. Sci. Math., 136(5):521-573, 2012.

[17] H. Federer. Geometric measure theory. Springer-Verlag, Berlin, Heidelberg, New York., 1969.

[18] W. H. Fleming. Flat chains over a finite coefficient Group. Trans. Amer. Math. Soc., 121:160-186, 1966.

[19] G. A. Francfort and S. Müller. Combined effects of homogenization and singular perturbations in elasticity. J. Reine Angew. Math., 454:1-35, 1994.

[20] M. Giaquinta, G. Modica, and J. Souček. Liquid crystals: Relaxed energies, dipoles, singular lines and singular points. Ann. Sc. Norm. Super. Pisa, Cl. Sci., IV. Ser., 17(3):415-437, 1990.

[21] M. Giaquinta, G. Modica, and J. Souček. Cartesian currents in the calculus of variations I. Cartesian currents. Ergebnisse der Mathematik und ihrer Grenzgebiete. 3. Folge. 37. Berlin: Springer, 1998.

[22] D. Gilbarg and N. S. Trudinger. Elliptic partial differential equations of second order. Reprint of the 1998 ed. Classics in Mathematics. Berlin: Springer., 2001.

[23] J. Hirth and J. Lothe. Theory of dislocations. Wiley, 2ed., New-York, 1982.

[24] R. Kupferman and C. Maor. The emergence of torsion in the continuum limit of distributed edge-dislocations. J. Geom. Mech., 7(3):361-387, 2015. 
[25] I. Mitrea and M. Mitrea. A remark on the regularity of the div-curl system. Proc. Am. Math. Soc., 137(5):1729-1733, 2009.

[26] S. Müller and M. Palombaro. Existence of minimizers for a polyconvex energy in a crystal with dislocations. Calc. Var., 31(4):473-482, 2008.

[27] W. Noll. Materially uniform simple bodies with inhomogeneities. Arch. Ration. Mech. Anal., 27:1-32, 1968.

[28] M. Ortiz. Lectures at the Vienna Summer School on Microstructures. Lectures at the Vienna Summer School on Microstructures, Vienna, 2000.

[29] R. S. Rivlin and D. W. Saunders. Large elastic deformations of isotropic materials vii. experiments on the deformation of rubber. Philosophical Transactions of the Royal Society of London A: Mathematical, Physical and Engineering Sciences, 243(865):251-288, 1951.

[30] A. Roychowdhury and A. Gupta. Non-metric connection and metric anomalies in materially uniform elastic solids. J. Elasticity, 126(1):1-26, 2017.

[31] R. Scala and N. Van Goethem. Constraint reaction and the Peach-Koehler force for dislocation networks. Mathematics and Mechanics of Complex Sytems, 4(2):105-138, 2016.

[32] R. Scala and N. Van Goethem. Currents and dislocations at the continuum scale. Methods Appl. Anal., 23(1):1-34, 2016.

[33] R. Scala and N. Van Goethem. Geometric and analytic properties of dislocation singularities. Proc. Roy. Soc. Edinburgh Sect. A, 149(4), 2019.

[34] R. Scala and N. Van Goethem. Variational evolution of dislocations in single crystals. J. Nonlinear Sci., 2019.

[35] N. Triantafyllidis and Elias C. Aifantis. A gradient approach to localization of deformation. I. Hyperelastic materials. J. Elasticity, 16:225-237, 1986.

[36] N. Van Goethem. Strain incompatibility in single crystals: Kröner's formula revisited. J. Elast., 103(1):95-111, 2011.

[37] N. Van Goethem. Incompatibility-governed singularities in linear elasticity with dislocations. Math. Mech. Solids, 22(8):1688-1695, 2017.

[38] N. Van Goethem and F. Dupret. A distributional approach to the geometry of $2 D$ dislocations at the continuum scale. Ann. Univ. Ferrara, 58(2):407-434, 2012.

[39] J. Van Schaftingen. Limiting fractional and Lorentz space estimates of differential forms. Proc. Am. Math. Soc., 138(1):235-240, 2010.

[40] B. White. Rectifiability of flat chains. Annals of Mathematics, 150:165-184, 1999.

[41] A. Yavari and A. Goriely. Riemann-Cartan geometry of nonlinear dislocation mechanics. Arch. Ration. Mech. Anal., 205(1):59-118, 2012.

[42] L. Zubov. Nonlinear Theory of Dislocations and Disclinations in Elastic Bodies. Lecture notes in physics, 47. Springer-Verlag, 1997. 